\title{
Theoretical study of the addition patterns of $\mathrm{C}_{60}$ fluorination: $\mathrm{C}_{60} \mathrm{~F}_{\mathrm{n}}(\mathrm{n}=1-60)$.
}

Gregory Van Lier, ${ }^{\mathrm{a}, \mathrm{b}}$ Montserrat Cases,,${ }^{\mathrm{c}}$ Christopher P. Ewels, ${ }^{\mathrm{d}, \mathrm{e}}$ Roger Taylor, ${ }^{\mathrm{f}}$ Paul Geerlings ${ }^{* \mathrm{~b}}$

Table of contents: Optimized geometries in Cartesian coordinates of the AM1 optimizations are provided for all structures discussed in detail in the paper and listed in Table 1.

$\mathrm{C}_{60}$

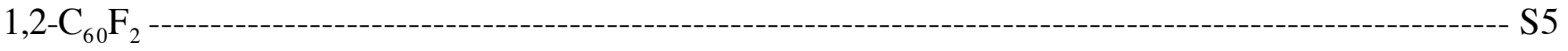

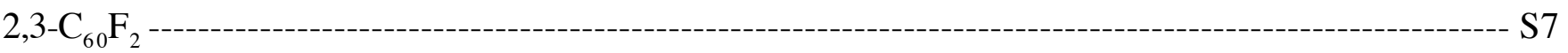

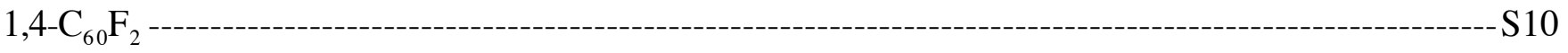

1,2,3,4-C ${ }_{60} \mathrm{~F}_{4}$-------------------------------------------------------------------------S12

$\mathrm{O}^{\prime}-\mathrm{C}_{60} \mathrm{~F}_{4}---$

$\mathrm{O}^{\prime}-\mathrm{C}_{60} \mathrm{~F}_{6}-\mathrm{-}-\mathrm{O}$

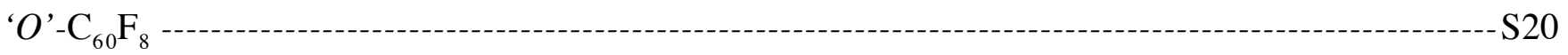

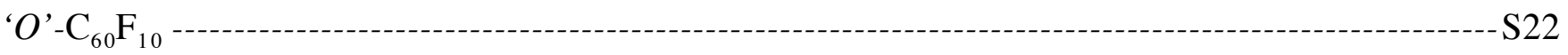

${ }^{\prime} \mathrm{O}^{\prime}-\mathrm{C}_{60} \mathrm{~F}_{12}-\mathrm{P}_{12}$

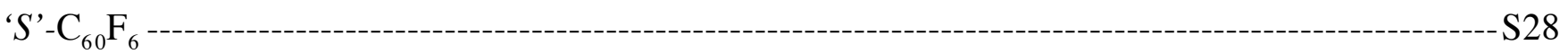

$\mathrm{S}^{\prime}-\mathrm{C}_{60} \mathrm{~F}_{8}$

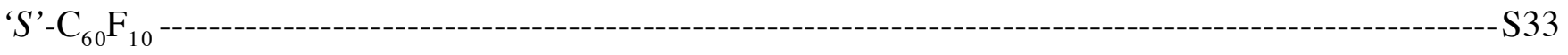

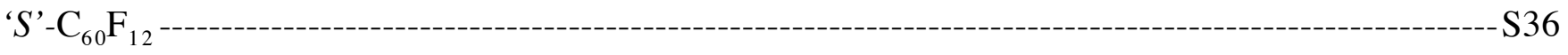

$' S$ '- $\mathrm{C}_{60} \mathrm{~F}_{14}$

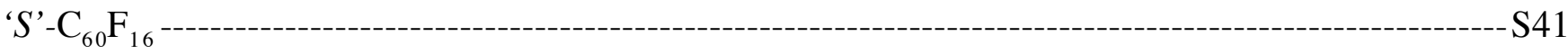

$' \mathrm{~S}^{\prime}-\mathrm{C}_{60} \mathrm{~F}_{18}$

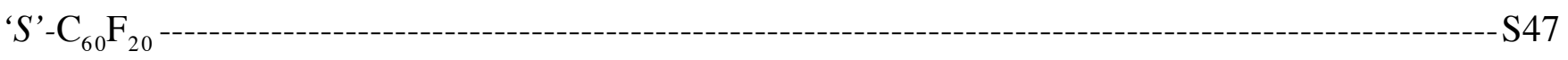

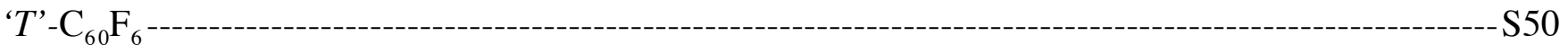

' $T$ ' $\mathrm{C}_{60} \mathrm{~F}_{8}$

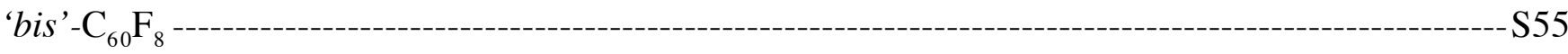

' $T$ ' $\mathrm{C}_{60} \mathrm{~F}_{10}$ 


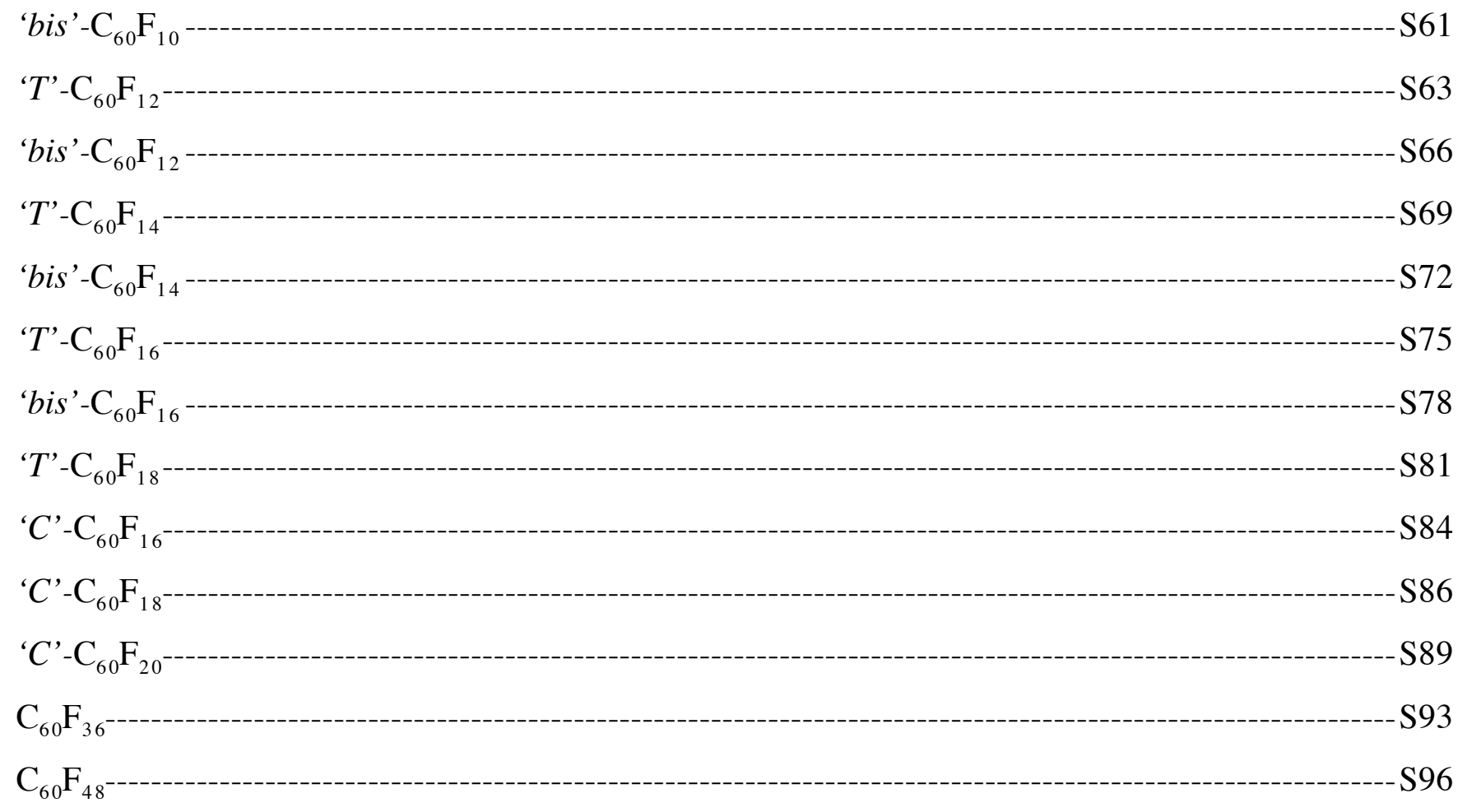


Supporting information:

Optimized geometries in Cartesian coordinates of the AM1 optimizations:

\begin{tabular}{|c|c|c|c|}
\hline $\mathrm{C}_{60}$ & & & \\
\hline $\bar{C}$ & -1.7562316885 & -3.0365001826 & 0.588940185 \\
\hline $\mathrm{C}$ & -1.9940571293 & -2.3045427499 & 1.834211417 \\
\hline $\bar{C}$ & -3.4305887252 & -0.7319454225 & 0.588940185 \\
\hline $\mathrm{C}$ & -2.8079479432 & -1.1843181452 & 1.834211417 \\
\hline $\bar{C}$ & -0.384805354 & -1.1843090977 & 3.3317859089 \\
\hline $\mathrm{C}$ & -2.4231347698 & 0.0000000032 & 2.6038217508 \\
\hline $\mathrm{C}$ & -0.7487898266 & -2.3045381117 & 2.6038217508 \\
\hline $\mathrm{C}$ & -1.2452562786 & 0.0000000016 & 3.3317859089 \\
\hline $\mathrm{C}$ & -2.8079479401 & 1.1843181527 & 1.834211417 \\
\hline $\bar{C}$ & 0.2586498556 & -3.0364916225 & 1.834211417 \\
\hline $\bar{C}$ & 1.0074334927 & 0.7319432745 & 3.3317859089 \\
\hline $\bar{C}$ & 1.0074334907 & -0.7319432772 & 3.3317859089 \\
\hline $\mathrm{C}$ & -1.9940571232 & 2.3045427552 & 1.834211417 \\
\hline $\mathrm{C}$ & -3.4305887232 & 0.7319454316 & 0.588940185 \\
\hline $\bar{C}$ & -1.7562316805 & 3.0365001873 & 0.588940185 \\
\hline $\bar{C}$ & -0.7487898205 & 2.3045381137 & 2.6038217508 \\
\hline $\mathrm{C}$ & 0.2586498637 & 3.0364916218 & 1.834211417 \\
\hline $\bar{C}$ & -0.3848053509 & 1.1843090987 & 3.3317859089 \\
\hline $\bar{C}$ & 2.9678023452 & -0.692336884 & 1.834211417 \\
\hline $\bar{C}$ & 1.5755528587 & -2.6086039007 & 1.834211417 \\
\hline $\bar{C}$ & -0.3639887439 & 3.4888673373 & 0.588940185 \\
\hline $\bar{C}$ & 1.5755528656 & 2.6086038965 & 1.834211417 \\
\hline $\mathrm{C}$ & 2.3451778545 & 2.6086057451 & 0.588940185 \\
\hline
\end{tabular}




\begin{tabular}{|c|c|c|c|}
\hline $\mathrm{C}$ & 1.9603572103 & 1.4242828794 & 2.6038217508 \\
\hline $\mathrm{C}$ & 2.967802347 & 0.6923368761 & 1.834211417 \\
\hline $\mathrm{C}$ & 2.3451778476 & -2.6086057513 & 0.588940185 \\
\hline $\mathrm{C}$ & -0.3639887531 & -3.4888673363 & 0.588940185 \\
\hline $\mathrm{C}$ & 1.9603572066 & -1.4242828846 & 2.6038217508 \\
\hline $\mathrm{C}$ & 3.2056313042 & -1.4242931735 & 0.588940185 \\
\hline $\mathrm{C}$ & 3.205631308 & 1.4242931651 & 0.588940185 \\
\hline $\mathrm{C}$ & -2.9678023452 & 0.692336884 & -1.834211417 \\
\hline $\mathrm{C}$ & 0.3639887531 & 3.4888673363 & -0.588940185 \\
\hline $\mathrm{C}$ & 0.3639887439 & -3.4888673373 & -0.588940185 \\
\hline $\mathrm{C}$ & -1.9603572103 & -1.4242828794 & -2.6038217508 \\
\hline $\mathrm{C}$ & -1.5755528587 & 2.6086039007 & -1.834211417 \\
\hline $\mathrm{C}$ & -3.205631308 & -1.4242931651 & -0.588940185 \\
\hline $\mathrm{C}$ & -2.3451778476 & 2.6086057513 & -0.588940185 \\
\hline $\mathrm{C}$ & -1.9603572066 & 1.4242828846 & -2.6038217508 \\
\hline $\mathrm{C}$ & -3.2056313042 & 1.4242931735 & -0.588940185 \\
\hline $\mathrm{C}$ & -2.3451778545 & -2.6086057451 & -0.588940185 \\
\hline $\mathrm{C}$ & 1.7562316805 & -3.0365001873 & -0.588940185 \\
\hline $\mathrm{C}$ & 1.9940571232 & -2.3045427552 & -1.834211417 \\
\hline $\mathrm{C}$ & 3.4305887232 & -0.7319454316 & -0.588940185 \\
\hline $\mathrm{C}$ & 2.8079479401 & -1.1843181527 & -1.834211417 \\
\hline $\mathrm{C}$ & 0.3848053509 & -1.1843090987 & -3.3317859089 \\
\hline $\mathrm{C}$ & 2.4231347698 & -0.0000000032 & -2.6038217508 \\
\hline $\mathrm{C}$ & 0.7487898205 & -2.3045381137 & -2.6038217508 \\
\hline $\mathrm{C}$ & 1.2452562786 & -0.0000000016 & -3.3317859089 \\
\hline $\mathrm{C}$ & 2.8079479432 & 1.1843181452 & -1.834211417 \\
\hline $\mathrm{C}$ & -0.2586498637 & -3.0364916218 & -1.834211417 \\
\hline
\end{tabular}




\begin{tabular}{|l|l|l|l|}
\hline $\mathrm{C}$ & -1.0074334907 & 0.7319432772 & -3.3317859089 \\
\hline $\mathrm{C}$ & -1.0074334927 & -0.7319432745 & -3.3317859089 \\
\hline $\mathrm{C}$ & 1.9940571293 & 2.3045427499 & -1.834211417 \\
\hline $\mathrm{C}$ & 3.4305887252 & 0.7319454225 & -0.588940185 \\
\hline $\mathrm{C}$ & 1.7562316885 & 3.0365001826 & -0.588940185 \\
\hline $\mathrm{C}$ & 0.7487898266 & 2.3045381117 & -2.6038217508 \\
\hline $\mathrm{C}$ & -0.2586498556 & 3.0364916225 & -1.834211417 \\
\hline $\mathrm{C}$ & 0.384805354 & 1.1843090977 & -3.3317859089 \\
\hline $\mathrm{C}$ & -2.967802347 & -0.6923368761 & -1.834211417 \\
\hline $\mathrm{C}$ & -1.5755528656 & -2.6086038965 & -1.834211417 \\
\hline
\end{tabular}

\begin{tabular}{|l|l|l|l|}
\hline $1,2-\mathrm{C}_{60} \mathrm{~F}_{2}$ & & & \\
\hline $\mathrm{C}$ & 3.31669243 & 1.50497357 & 0.80811443 \\
\hline $\mathrm{C}$ & 3.07710720 & 0.77183171 & 2.05205320 \\
\hline $\mathrm{C}$ & 3.31385825 & -1.34407005 & 0.80585627 \\
\hline $\mathrm{C}$ & 3.07635396 & -0.61322644 & 2.05110751 \\
\hline $\mathrm{C}$ & 1.11630415 & 0.81100785 & 3.54941057 \\
\hline $\mathrm{C}$ & 2.06876442 & -1.34535606 & 2.82086234 \\
\hline $\mathrm{C}$ & 2.06914254 & 1.50335410 & 2.82148183 \\
\hline $\mathrm{C}$ & 1.11627318 & -0.65293909 & 3.55004716 \\
\hline $\mathrm{C}$ & 1.68325446 & -2.52888892 & 2.05156792 \\
\hline $\mathrm{C}$ & 1.68480170 & 2.68803865 & 2.05210175 \\
\hline $\mathrm{C}$ & -1.13633863 & 0.07914583 & 3.55259251 \\
\hline $\mathrm{C}$ & -0.27603127 & 1.26326861 & 3.55009780 \\
\hline $\mathrm{C}$ & 0.36707951 & -2.95841514 & 2.05491148 \\
\hline $\mathrm{C}$ & 2.44918788 & -2.52621292 & 0.80317905 \\
\hline
\end{tabular}




\begin{tabular}{|c|c|c|c|}
\hline $\mathrm{C}$ & -0.25805000 & -3.40413289 & 0.80804951 \\
\hline $\mathrm{C}$ & -0.64000701 & -2.22672191 & 2.82558668 \\
\hline $\mathrm{C}$ & -1.88405702 & -2.22383674 & 2.05414043 \\
\hline $\mathrm{C}$ & -0.27571076 & -1.10532373 & 3.55256488 \\
\hline $\mathrm{C}$ & -1.88507935 & 2.38213845 & 2.05170110 \\
\hline $\mathrm{C}$ & 0.36726939 & 3.11523782 & 2.05120454 \\
\hline $\mathrm{C}$ & -1.64147530 & -2.94755201 & 0.80463805 \\
\hline $\mathrm{C}$ & -2.69706560 & -1.10490439 & 2.05416895 \\
\hline $\mathrm{C}$ & -3.31046518 & -0.65055913 & 0.80470093 \\
\hline $\mathrm{C}$ & -2.31544505 & 0.07915075 & 2.82565164 \\
\hline $\mathrm{C}$ & -2.70022410 & 1.26305265 & 2.05502976 \\
\hline $\mathrm{C}$ & -0.25447627 & 3.56695680 & 0.80598803 \\
\hline $\mathrm{C}$ & 2.45606648 & 2.68944452 & 0.80814590 \\
\hline $\mathrm{C}$ & -0.64031896 & 2.38310906 & 2.82096176 \\
\hline $\mathrm{C}$ & -1.64593165 & 3.10981259 & 0.80333317 \\
\hline $\mathrm{C}$ & -3.31729026 & 0.80625002 & 0.80816653 \\
\hline $\mathrm{C}$ & 2.08707358 & -2.21157432 & -1.61730490 \\
\hline $\mathrm{C}$ & -2.21477162 & -2.51022793 & -0.38098851 \\
\hline $\mathrm{C}$ & 1.86773568 & 3.11871880 & -0.37012971 \\
\hline $\mathrm{C}$ & 2.52400484 & 0.07916238 & -2.37762136 \\
\hline $\mathrm{C}$ & -0.16799182 & -2.94316074 & -1.61507165 \\
\hline $\mathrm{C}$ & 3.54232031 & -0.65123409 & -0.37083761 \\
\hline $\mathrm{C}$ & 0.46208932 & -3.39231759 & -0.37588778 \\
\hline $\mathrm{C}$ & 0.84800181 & -2.20492081 & -2.39124977 \\
\hline $\mathrm{C}$ & 1.85399642 & -2.94523365 & -0.37444802 \\
\hline $\mathrm{C}$ & 3.54317591 & 0.81284772 & -0.37019030 \\
\hline $\mathrm{C}$ & 0.47501375 & 3.57023157 & -0.37072520 \\
\hline
\end{tabular}




\begin{tabular}{|c|c|c|c|}
\hline $\bar{C}$ & -0.14543252 & 3.11098574 & -1.61501443 \\
\hline $\mathrm{C}$ & -2.22836770 & 2.67323705 & -0.37429444 \\
\hline $\mathrm{C}$ & -1.45862225 & 2.66829420 & -1.61717778 \\
\hline $\bar{C}$ & 0.47792112 & 1.24833247 & -3.09271196 \\
\hline $\mathrm{C}$ & -1.83512078 & 1.48781459 & -2.39115292 \\
\hline $\mathrm{C}$ & 0.85501551 & 2.37615566 & -2.37755741 \\
\hline $\mathrm{C}$ & -0.90306883 & 0.80751223 & -3.12471912 \\
\hline $\mathrm{C}$ & -2.85111433 & 0.74957471 & -1.61497465 \\
\hline $\mathrm{C}$ & 2.10421865 & 2.38379349 & -1.61381724 \\
\hline $\mathrm{C}$ & 0.48891740 & -1.10826360 & -3.12476948 \\
\hline $\mathrm{C}$ & 1.33483486 & 0.06897981 & -3.09274279 \\
\hline $\mathrm{C}$ & -2.86445441 & -0.61766466 & -1.62630380 \\
\hline $\mathrm{C}$ & -3.08360275 & 1.48755367 & -0.37575313 \\
\hline $\mathrm{C}$ & -3.07168449 & -1.33087464 & -0.38095593 \\
\hline $\mathrm{C}$ & -2.07513717 & -1.50771674 & -2.61239277 \\
\hline $\mathrm{C}$ & -1.47246571 & -2.53343871 & -1.62634926 \\
\hline $\mathrm{C}$ & -0.96232690 & -0.69912957 & -3.46253445 \\
\hline $\mathrm{C}$ & 2.91381329 & -1.09939314 & -1.61512037 \\
\hline $\mathrm{C}$ & 2.91722731 & 1.26486120 & -1.61384560 \\
\hline $\bar{F}$ & -2.90994261 & -2.11425678 & -3.53092143 \\
\hline $\mathrm{F}$ & -1.25364913 & -0.91076899 & -4.79626079 \\
\hline
\end{tabular}

\begin{tabular}{|l|l|l|l|}
\hline $2,3-\mathrm{C}_{60} \mathrm{~F}_{2}$ & & & \\
\hline $\mathrm{C}$ & -1.733338 & 1.425546 & 2.90341 \\
\hline $\mathrm{C}$ & -2.858504 & 0.693384 & 2.321484 \\
\hline $\mathrm{C}$ & -2.8585 & -0.692705 & 2.321682 \\
\hline
\end{tabular}




\begin{tabular}{|c|c|c|c|}
\hline $\mathrm{C}$ & -1.733335 & -1.424696 & 2.903818 \\
\hline $\mathrm{C}$ & -0.669595 & -0.731392 & 3.455407 \\
\hline $\mathrm{C}$ & -0.669601 & 0.732393 & 3.455179 \\
\hline $\mathrm{C}$ & -1.487038 & 2.609923 & 2.076503 \\
\hline $\mathrm{C}$ & -2.465012 & 2.611441 & 0.986815 \\
\hline $\mathrm{C}$ & -3.310071 & 1.424944 & 1.137325 \\
\hline $\mathrm{C}$ & -3.735991 & 0.731661 & 0.016706 \\
\hline $\mathrm{C}$ & -3.310038 & -1.424594 & 1.137721 \\
\hline $\mathrm{C}$ & -1.487044 & -2.609317 & 2.077259 \\
\hline $\mathrm{C}$ & -0.190946 & -3.034621 & 1.84188 \\
\hline $\mathrm{C}$ & 0.934955 & -2.292189 & 2.410329 \\
\hline $\mathrm{C}$ & 0.701412 & -1.177742 & 3.197812 \\
\hline $\mathrm{C}$ & 1.53957 & 0.000431 & 3.026379 \\
\hline $\mathrm{C}$ & 0.701416 & 1.178657 & 3.197482 \\
\hline $\mathrm{C}$ & 0.934979 & 2.292895 & 2.409692 \\
\hline $\mathrm{C}$ & -0.190923 & 3.035153 & 1.841012 \\
\hline $\mathrm{C}$ & -2.093933 & 3.043249 & -0.27481 \\
\hline $\mathrm{C}$ & -0.72427 & 3.501618 & -0.520298 \\
\hline $\mathrm{C}$ & 0.204185 & 3.497009 & 0.510195 \\
\hline $\mathrm{C}$ & 1.571743 & 3.048013 & 0.26226 \\
\hline $\mathrm{C}$ & 2.026108 & 2.282654 & 1.442218 \\
\hline $\mathrm{C}$ & 2.834422 & 1.18117 & 1.296922 \\
\hline $\mathrm{C}$ & 2.56129 & 0.000284 & 2.075751 \\
\hline $\mathrm{C}$ & 2.834395 & -1.180816 & 1.29724 \\
\hline $\mathrm{C}$ & 2.026115 & -2.28227 & 1.442872 \\
\hline $\mathrm{C}$ & -2.465005 & -2.611139 & 0.987559 \\
\hline $\mathrm{C}$ & 0.971209 & -2.619325 & -2.073174 \\
\hline
\end{tabular}




\begin{tabular}{|c|c|c|c|}
\hline $\mathrm{C}$ & -0.32935 & -3.051276 & -1.851787 \\
\hline $\mathrm{C}$ & -1.448589 & -2.312896 & -2.432161 \\
\hline $\mathrm{C}$ & -1.208846 & -1.18647 & -3.20541 \\
\hline $\mathrm{C}$ & 0.162294 & -0.739586 & -3.43038 \\
\hline $\mathrm{C}$ & 2.360513 & -0.717569 & -2.253688 \\
\hline $\mathrm{C}$ & 2.804129 & -1.421355 & -1.150736 \\
\hline $\mathrm{C}$ & 1.946104 & -2.614602 & -0.984054 \\
\hline $\mathrm{C}$ & 1.571747 & -3.047954 & 0.263108 \\
\hline $\mathrm{C}$ & 0.204167 & -3.496856 & 0.511175 \\
\hline $\mathrm{C}$ & -0.724275 & -3.501726 & -0.519303 \\
\hline $\mathrm{C}$ & -2.543384 & -2.309086 & -1.45889 \\
\hline $\mathrm{C}$ & -3.339297 & -1.185485 & -1.317329 \\
\hline $\mathrm{C}$ & -3.091228 & -0.000311 & -2.141063 \\
\hline $\mathrm{C}$ & -2.05487 & -0.000433 & -3.058319 \\
\hline $\mathrm{C}$ & -1.208856 & 1.185544 & -3.205737 \\
\hline $\mathrm{C}$ & 0.162286 & 0.738572 & -3.430588 \\
\hline $\mathrm{C}$ & 1.214862 & 1.427057 & -2.876849 \\
\hline $\mathrm{C}$ & 2.36049 & 0.716894 & -2.253867 \\
\hline $\mathrm{C}$ & 3.582172 & -0.830445 & 0.000566 \\
\hline $\mathrm{C}$ & 3.582192 & 0.830457 & 0.000336 \\
\hline $\mathrm{C}$ & 2.80419 & 1.42104 & -1.151141 \\
\hline $\mathrm{C}$ & 1.946154 & 2.614333 & -0.984783 \\
\hline $\mathrm{C}$ & 0.97122 & 2.618729 & -2.073904 \\
\hline $\mathrm{C}$ & -0.329345 & 3.050746 & -1.852638 \\
\hline $\mathrm{C}$ & -1.448563 & 2.312125 & -2.432755 \\
\hline $\mathrm{C}$ & -2.543404 & 2.308677 & -1.459555 \\
\hline $\mathrm{C}$ & -3.339325 & 1.185105 & -1.317644 \\
\hline
\end{tabular}




\begin{tabular}{|l|l|l|l|}
\hline C & -2.093934 & -3.043317 & -0.273951 \\
\hline C & -3.735963 & -0.731645 & 0.016896 \\
\hline C & 1.214889 & -1.427882 & -2.876435 \\
\hline F & 4.897735 & 1.24108 & 0.011923 \\
\hline F & 4.897707 & -1.241082 & 0.012245 \\
\hline
\end{tabular}

\begin{tabular}{|c|c|c|c|}
\hline $1,4-\mathrm{C}_{60} \mathrm{~F}_{2}$ & & & \\
\hline $\mathrm{C}$ & 3.496659 & 1.424042 & -0.018654 \\
\hline $\mathrm{C}$ & 3.494994 & 0.692367 & 1.248846 \\
\hline $\mathrm{C}$ & 3.49499 & -0.692405 & 1.24883 \\
\hline $\bar{C}$ & 3.49665 & -1.424032 & -0.0187 \\
\hline $\mathrm{C}$ & 3.500195 & -0.731817 & -1.218586 \\
\hline $\mathrm{C}$ & 3.500214 & 0.731875 & -1.218566 \\
\hline $\mathrm{C}$ & 2.650952 & 2.607513 & 0.140836 \\
\hline $\mathrm{C}$ & 2.124091 & 2.606174 & 1.507765 \\
\hline $\mathrm{C}$ & 2.649269 & 1.424031 & 2.193854 \\
\hline $\mathrm{C}$ & 1.853298 & 0.732417 & 3.090509 \\
\hline $\bar{C}$ & 2.649303 & -1.424102 & 2.193848 \\
\hline $\mathrm{C}$ & 2.650939 & -2.607495 & 0.14075 \\
\hline $\mathrm{C}$ & 1.855973 & -3.036406 & -0.908076 \\
\hline $\bar{C}$ & 1.858624 & -2.305034 & -2.176225 \\
\hline $\bar{C}$ & 2.658804 & -1.184251 & -2.327632 \\
\hline $\mathrm{C}$ & 2.138565 & 0.000051 & -3.012775 \\
\hline $\mathrm{C}$ & 2.658812 & 1.184325 & -2.327587 \\
\hline $\mathrm{C}$ & 1.858632 & 2.305093 & -2.17615 \\
\hline $\mathrm{C}$ & 1.855986 & 3.036435 & -0.907982 \\
\hline
\end{tabular}




\begin{tabular}{|c|c|c|c|}
\hline $\mathrm{C}$ & 0.828571 & 3.028396 & 1.751234 \\
\hline $\mathrm{C}$ & -0.017324 & 3.472042 & 0.642012 \\
\hline $\mathrm{C}$ & 0.484895 & 3.481921 & -0.649923 \\
\hline $\mathrm{C}$ & -0.357338 & 3.024802 & -1.746803 \\
\hline $\mathrm{C}$ & 0.490983 & 2.301842 & -2.69685 \\
\hline $\mathrm{C}$ & -0.000702 & 1.183257 & -3.344362 \\
\hline $\mathrm{C}$ & 0.844877 & 0.000053 & -3.509047 \\
\hline $\mathrm{C}$ & -0.000694 & -1.183184 & -3.344423 \\
\hline $\mathrm{C}$ & 0.490984 & -2.30182 & -2.696961 \\
\hline $\mathrm{C}$ & 2.124075 & -2.606174 & 1.507675 \\
\hline $\mathrm{C}$ & -2.189892 & -2.620056 & -0.144596 \\
\hline $\mathrm{C}$ & -1.383486 & -3.02326 & 0.889304 \\
\hline $\mathrm{C}$ & -1.378082 & -2.291869 & 2.174391 \\
\hline $\mathrm{C}$ & -2.159705 & -1.172777 & 2.304883 \\
\hline $\mathrm{C}$ & -3.013229 & -0.686411 & 1.196364 \\
\hline $\mathrm{C}$ & -3.039728 & -0.683297 & -1.259596 \\
\hline $\mathrm{C}$ & -2.16666 & -1.404179 & -2.163707 \\
\hline $\mathrm{C}$ & -1.650374 & -2.588001 & -1.485976 \\
\hline $\mathrm{C}$ & -0.35733 & -3.024774 & -1.746871 \\
\hline $\mathrm{C}$ & 0.484907 & -3.481842 & -0.650001 \\
\hline $\mathrm{C}$ & -0.01733 & -3.471957 & 0.64193 \\
\hline $\mathrm{C}$ & -0.015533 & -2.300551 & 2.698911 \\
\hline $\mathrm{C}$ & 0.484617 & -1.183567 & 3.353 \\
\hline $\mathrm{C}$ & -0.355371 & -0.000066 & 3.514957 \\
\hline $\mathrm{C}$ & -1.644695 & -0.000053 & 3.001265 \\
\hline $\mathrm{C}$ & -2.159631 & 1.172712 & 2.304915 \\
\hline $\mathrm{C}$ & -3.013233 & 0.686443 & 1.196377 \\
\hline
\end{tabular}




\begin{tabular}{|l|l|l|l|}
\hline C & -3.317836 & 1.556364 & -0.005139 \\
\hline C & -3.039836 & 0.683377 & -1.259627 \\
\hline C & -1.365073 & -0.727121 & -3.071431 \\
\hline C & -1.365079 & 0.727164 & -3.071408 \\
\hline C & -2.166688 & 1.404215 & -2.163679 \\
\hline C & -1.650392 & 2.588041 & -1.485948 \\
\hline C & -2.189869 & 2.619953 & -0.14454 \\
\hline C & -1.383431 & 3.023153 & 0.889334 \\
\hline C & -1.378054 & 2.291806 & 2.174396 \\
\hline C & -0.015538 & 2.300436 & 2.698969 \\
\hline C & 0.484599 & 1.18344 & 3.353047 \\
\hline C & 0.828592 & -3.028447 & 1.751165 \\
\hline C & 1.853315 & -0.732532 & 3.090504 \\
\hline C & -3.317857 & -1.556361 & -0.005194 \\
\hline F & -4.579138 & 2.105352 & 0.019129 \\
\hline F & -4.579112 & -2.105281 & 0.018969 \\
\hline
\end{tabular}

\begin{tabular}{|l|l|l|l|}
\hline $1,2,3,4-\mathrm{C}_{60} \mathrm{~F}_{4}$ & & & \\
\hline $\mathrm{C}$ & 3.26527725 & 1.61930943 & 1.00997384 \\
\hline $\mathrm{C}$ & 3.02729584 & 0.89126671 & 2.25822323 \\
\hline $\mathrm{C}$ & 3.27084432 & -1.22752680 & 1.01817012 \\
\hline $\mathrm{C}$ & 3.03150233 & -0.49316105 & 2.26262451 \\
\hline $\mathrm{C}$ & 1.06209567 & 0.93186118 & 3.75000976 \\
\hline $\mathrm{C}$ & 2.02078358 & -1.22432269 & 3.02948062 \\
\hline $\mathrm{C}$ & 2.01669165 & 1.62346530 & 3.02298606 \\
\hline $\mathrm{C}$ & 1.06349845 & -0.53185187 & 3.75324641 \\
\hline
\end{tabular}




\begin{tabular}{|c|c|c|c|}
\hline $\mathrm{C}$ & 1.63745126 & -2.40597915 & 2.25597308 \\
\hline $\mathrm{C}$ & 1.63284936 & 2.80661127 & 2.25118811 \\
\hline $\mathrm{C}$ & -1.19066374 & 0.19923026 & 3.74745213 \\
\hline $\mathrm{C}$ & -0.33044134 & 1.38349702 & 3.74643044 \\
\hline $\mathrm{C}$ & 0.32115154 & -2.83131678 & 2.25195526 \\
\hline $\mathrm{C}$ & 2.40640135 & -2.40047027 & 1.00947705 \\
\hline $\mathrm{C}$ & -0.29476806 & -3.25780127 & 0.99486718 \\
\hline $\mathrm{C}$ & -0.69057896 & -2.10531486 & 3.02197709 \\
\hline $\mathrm{C}$ & -1.93223178 & -2.10049023 & 2.24616311 \\
\hline $\mathrm{C}$ & -0.32887090 & -0.98427342 & 3.75116687 \\
\hline $\mathrm{C}$ & -1.93842035 & 2.50177478 & 2.24545527 \\
\hline $\mathrm{C}$ & 0.31474334 & 3.23410568 & 2.24779802 \\
\hline $\mathrm{C}$ & -1.67802103 & -2.80917909 & 0.99131195 \\
\hline $\mathrm{C}$ & -2.74834172 & -0.98355789 & 2.24470107 \\
\hline $\mathrm{C}$ & -3.36187836 & -0.52967339 & 0.99465098 \\
\hline $\mathrm{C}$ & -2.36876885 & 0.19932064 & 3.01819717 \\
\hline $\mathrm{C}$ & -2.75438726 & 1.38335177 & 2.24775180 \\
\hline $\mathrm{C}$ & -0.30575199 & 3.68575136 & 1.00195418 \\
\hline $\mathrm{C}$ & 2.40609998 & 2.80622773 & 1.00892728 \\
\hline $\mathrm{C}$ & -0.69402324 & 2.50262120 & 3.01601753 \\
\hline $\mathrm{C}$ & -1.69799628 & 3.22902430 & 0.99721259 \\
\hline $\mathrm{C}$ & -3.37338584 & 0.92736615 & 1.00109016 \\
\hline $\mathrm{C}$ & 2.08050897 & -2.11735469 & -1.42883705 \\
\hline $\mathrm{C}$ & -2.23385138 & -2.36190612 & -0.19504178 \\
\hline $\mathrm{C}$ & 1.81967177 & 3.23582693 & -0.16933082 \\
\hline $\mathrm{C}$ & 2.46575012 & 0.18516875 & -2.17608580 \\
\hline $\mathrm{C}$ & -0.18603111 & -2.78323756 & -1.43373107 \\
\hline
\end{tabular}




\begin{tabular}{|c|c|c|c|}
\hline $\mathrm{C}$ & 3.48905103 & -0.54121485 & -0.16597135 \\
\hline $\mathrm{C}$ & 0.42328667 & -3.22368438 & -0.18820971 \\
\hline $\mathrm{C}$ & 0.91304606 & -2.36749518 & -2.41921084 \\
\hline $\mathrm{C}$ & 1.81670448 & -2.80460868 & -0.18089252 \\
\hline $\mathrm{C}$ & 3.48663869 & 0.92114763 & -0.16516494 \\
\hline $\mathrm{C}$ & 0.42666043 & 3.68761841 & -0.17291146 \\
\hline $\mathrm{C}$ & -0.19279787 & 3.21761834 & -1.41403976 \\
\hline $\mathrm{C}$ & -2.28151390 & 2.79190754 & -0.17999390 \\
\hline $\mathrm{C}$ & -1.50550609 & 2.77798027 & -1.41889176 \\
\hline $\mathrm{C}$ & 0.43400269 & 1.31557039 & -2.85081854 \\
\hline $\mathrm{C}$ & -1.87756554 & 1.59381628 & -2.18725475 \\
\hline $\mathrm{C}$ & 0.80559128 & 2.46676378 & -2.16107916 \\
\hline $\mathrm{C}$ & -0.93991143 & 0.88771133 & -2.89270703 \\
\hline $\mathrm{C}$ & -2.91088947 & 0.87120631 & -1.42307612 \\
\hline $\mathrm{C}$ & 2.05113347 & 2.48985195 & -1.40826863 \\
\hline $\mathrm{C}$ & 0.52489132 & -1.08710502 & -3.30068959 \\
\hline $\mathrm{C}$ & 1.29544856 & 0.16271376 & -2.88694907 \\
\hline $\mathrm{C}$ & -2.92051720 & -0.49539268 & -1.44169873 \\
\hline $\mathrm{C}$ & -3.14173773 & 1.60931878 & -0.18301854 \\
\hline $\mathrm{C}$ & -3.11608727 & -1.20477515 & -0.19357658 \\
\hline $\mathrm{C}$ & -2.11748027 & -1.38460940 & -2.42700376 \\
\hline $\mathrm{C}$ & -1.47622205 & -2.36479735 & -1.43704335 \\
\hline $\mathrm{C}$ & -1.04765088 & -0.57708337 & -3.30472708 \\
\hline $\mathrm{C}$ & 2.87470947 & -1.00521106 & -1.40819621 \\
\hline $\mathrm{C}$ & 2.85594196 & 1.36345052 & -1.40767863 \\
\hline $\mathrm{F}$ & -2.95830455 & -2.03034635 & -3.31496490 \\
\hline $\mathrm{F}$ & -1.47056562 & -0.68916589 & -4.61811295 \\
\hline
\end{tabular}




\begin{tabular}{|l|l|l|l|}
\hline $\mathrm{F}$ & 1.21895041 & -3.38513939 & -3.30422475 \\
\hline $\mathrm{F}$ & 0.80763244 & -1.42805605 & -4.61225364 \\
\hline
\end{tabular}

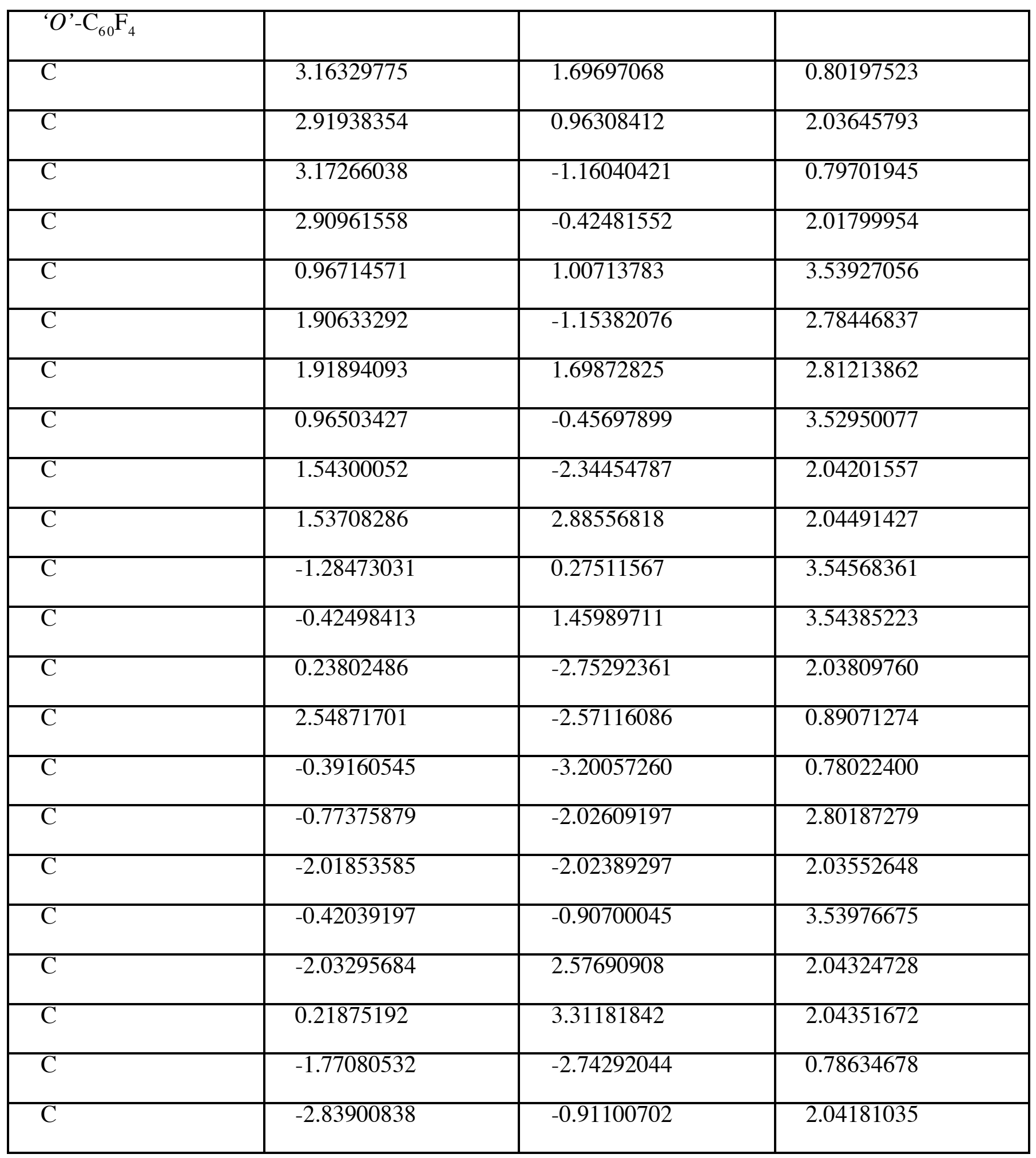




\begin{tabular}{|c|c|c|c|}
\hline $\mathrm{C}$ & -3.45806869 & -0.45580031 & 0.79419279 \\
\hline $\mathrm{C}$ & -2.46209001 & 0.27351154 & 2.81609225 \\
\hline $\mathrm{C}$ & -2.84757700 & 1.45684399 & 2.04611293 \\
\hline $\mathrm{C}$ & -0.40245668 & 3.76165918 & 0.79761164 \\
\hline $\bar{C}$ & 2.30834804 & 2.88590820 & 0.80071874 \\
\hline $\mathrm{C}$ & -0.78902438 & 2.57955110 & 2.81341699 \\
\hline $\mathrm{C}$ & -1.79374698 & 3.30427483 & 0.79480226 \\
\hline $\mathrm{C}$ & -3.46520021 & 1.00063892 & 0.79915740 \\
\hline $\bar{C}$ & 1.94431568 & -2.03032316 & -1.62761804 \\
\hline $\mathrm{C}$ & -2.35823981 & -2.30935801 & -0.39538114 \\
\hline $\mathrm{C}$ & 1.71930528 & 3.31178578 & -0.37832283 \\
\hline $\mathrm{C}$ & 2.36310583 & 0.26085117 & -2.37179141 \\
\hline $\mathrm{C}$ & -0.30436547 & -2.73450381 & -1.60312426 \\
\hline $\mathrm{C}$ & 3.38190615 & -0.46852405 & -0.36369795 \\
\hline $\mathrm{C}$ & 0.32234458 & -3.20887821 & -0.38850168 \\
\hline $\mathrm{C}$ & 0.70584510 & -2.00046560 & -2.37488207 \\
\hline $\mathrm{C}$ & 1.85464699 & -3.06382063 & -0.48638448 \\
\hline $\mathrm{C}$ & 3.38086826 & 0.99273245 & -0.37209199 \\
\hline $\mathrm{C}$ & 0.32740429 & 3.76384111 & -0.37944317 \\
\hline $\mathrm{C}$ & -0.29354631 & 3.30521785 & -1.62385646 \\
\hline $\mathrm{C}$ & -2.37657318 & 2.86812192 & -0.38293421 \\
\hline $\bar{C}$ & -1.60758161 & 2.86385373 & -1.62618094 \\
\hline $\mathrm{C}$ & 0.32653282 & 1.44482789 & -3.10434097 \\
\hline $\mathrm{C}$ & -1.98595571 & 1.68481940 & -2.40139795 \\
\hline $\mathrm{C}$ & 0.70557851 & 2.56957771 & -2.38666266 \\
\hline $\mathrm{C}$ & -1.05477748 & 1.00463051 & -3.13629959 \\
\hline $\mathrm{C}$ & -3.00208531 & 0.94648197 & -1.62511621 \\
\hline
\end{tabular}




\begin{tabular}{|l|l|l|l|}
\hline C & 1.95432968 & 2.57191566 & -1.62010308 \\
\hline C & 0.33709764 & -0.91011857 & -3.12188678 \\
\hline C & 1.17859820 & 0.26057072 & -3.09738212 \\
\hline C & -3.01651471 & -0.42080182 & -1.63761225 \\
\hline C & -3.23258110 & 1.68310052 & -0.38474731 \\
\hline C & -3.22333217 & -1.13456622 & -0.39238856 \\
\hline C & -2.22208074 & -1.31097441 & -2.62137550 \\
\hline C & -1.61476473 & -2.32837692 & -1.63074356 \\
\hline C & -1.11199276 & -0.50436505 & -3.46943753 \\
\hline C & 2.74653547 & -0.92034694 & -1.61718574 \\
\hline F & 2.75905062 & 1.44758509 & -1.61435341 \\
\hline F & -3.05449971 & -1.92512319 & -3.53693642 \\
\hline F & -1.39580544 & -0.71988833 & -4.80411043 \\
\hline F & 3.49251078 & -3.52462279 & 1.21924991 \\
\hline
\end{tabular}

\begin{tabular}{|l|l|l|l|}
\hline${ }^{\prime}{ }^{\prime}-\mathrm{C}_{60} \mathrm{~F}_{6}$ & & & \\
\hline $\mathrm{C}$ & 2.99816009 & 1.55639370 & 0.89393423 \\
\hline $\mathrm{C}$ & 2.75033097 & 0.83227251 & 2.12952615 \\
\hline $\mathrm{C}$ & 3.00100500 & -1.28510928 & 0.90758169 \\
\hline $\mathrm{C}$ & 2.74505115 & -0.55705523 & 2.12559679 \\
\hline $\mathrm{C}$ & 0.80844994 & 0.87952460 & 3.64630651 \\
\hline $\mathrm{C}$ & 1.74641516 & -1.28546540 & 2.90052806 \\
\hline $\mathrm{C}$ & 1.75527577 & 1.56707270 & 2.90936470 \\
\hline $\mathrm{C}$ & 0.80585274 & -0.58553251 & 3.64255486 \\
\hline $\mathrm{C}$ & 1.38232720 & -2.47642040 & 2.15862897 \\
\hline
\end{tabular}




\begin{tabular}{|c|c|c|c|}
\hline $\mathrm{C}$ & 1.37390284 & 2.74955059 & 2.13939664 \\
\hline $\mathrm{C}$ & -1.44346779 & 0.14825997 & 3.65953590 \\
\hline $\mathrm{C}$ & -0.58406480 & 1.33257173 & 3.65647322 \\
\hline $\mathrm{C}$ & 0.07685644 & -2.88351560 & 2.15467565 \\
\hline $\mathrm{C}$ & 2.38648455 & -2.69898380 & 1.00358650 \\
\hline $\mathrm{C}$ & -0.55318229 & -3.33164654 & 0.89697467 \\
\hline $\mathrm{C}$ & -0.93403695 & -2.15435862 & 2.91736021 \\
\hline $\mathrm{C}$ & -2.17859349 & -2.15148232 & 2.15080268 \\
\hline $\mathrm{C}$ & -0.57958531 & -1.03437661 & 3.65403766 \\
\hline $\mathrm{C}$ & -2.19507998 & 2.45054028 & 2.15943819 \\
\hline $\mathrm{C}$ & 0.05626231 & 3.18121107 & 2.15280373 \\
\hline $\mathrm{C}$ & -1.93184974 & -2.87153299 & 0.90226214 \\
\hline $\mathrm{C}$ & -2.99879796 & -1.03814913 & 2.15687877 \\
\hline $\mathrm{C}$ & -3.61839280 & -0.58331611 & 0.90962386 \\
\hline $\mathrm{C}$ & -2.62197101 & 0.14644328 & 2.93095725 \\
\hline $\mathrm{C}$ & -3.00935168 & 1.32953290 & 2.16192305 \\
\hline $\mathrm{C}$ & -0.55967645 & 3.62229305 & 0.90897841 \\
\hline $\mathrm{C}$ & 2.13758323 & 2.75598058 & 0.89360044 \\
\hline $\mathrm{C}$ & -0.95026029 & 2.45319080 & 2.92858844 \\
\hline $\mathrm{C}$ & -1.95454265 & 3.17421822 & 0.90928258 \\
\hline $\mathrm{C}$ & -3.62742696 & 0.87302046 & 0.91511405 \\
\hline $\mathrm{C}$ & 1.77560303 & -2.14946675 & -1.50415708 \\
\hline $\mathrm{C}$ & -2.51869270 & -2.43753729 & -0.27991297 \\
\hline $\mathrm{C}$ & 1.56819425 & 3.18905186 & -0.27183729 \\
\hline $\mathrm{C}$ & 2.21304303 & 0.13956624 & -2.26239679 \\
\hline $\mathrm{C}$ & -0.46580353 & -2.86667587 & -1.48676666 \\
\hline $\mathrm{C}$ & 3.20135000 & -0.57779781 & -0.25061662 \\
\hline
\end{tabular}




\begin{tabular}{|c|c|c|c|}
\hline $\mathrm{C}$ & 0.16059079 & -3.34160475 & -0.27163150 \\
\hline $\mathrm{C}$ & 0.54659552 & -2.12618584 & -2.25011154 \\
\hline $\mathrm{C}$ & 1.69348558 & -3.18987927 & -0.36982765 \\
\hline $\mathrm{C}$ & 3.23097705 & 0.86903734 & -0.26734063 \\
\hline $\mathrm{C}$ & 0.18026590 & 3.60734936 & -0.26466230 \\
\hline $\mathrm{C}$ & -0.43732425 & 3.14807845 & -1.50403503 \\
\hline $\mathrm{C}$ & -2.53655805 & 2.73784720 & -0.26678117 \\
\hline $\mathrm{C}$ & -1.76058147 & 2.72468151 & -1.50609316 \\
\hline $\mathrm{C}$ & 0.17458929 & 1.32722132 & -2.97545070 \\
\hline $\mathrm{C}$ & -2.14285955 & 1.55544297 & -2.28017102 \\
\hline $\mathrm{C}$ & 0.55564892 & 2.44563513 & -2.27845928 \\
\hline $\mathrm{C}$ & -1.20104318 & 0.87886458 & -3.00848671 \\
\hline $\mathrm{C}$ & -3.16435053 & 0.81863900 & -1.50994347 \\
\hline $\mathrm{C}$ & 1.95919692 & 2.70242444 & -1.68686215 \\
\hline $\mathrm{C}$ & 0.18433984 & -1.02612952 & -2.99141130 \\
\hline $\mathrm{C}$ & 1.03183571 & 0.13514963 & -2.96639326 \\
\hline $\mathrm{C}$ & -3.17578463 & -0.54873743 & -1.52198484 \\
\hline $\mathrm{C}$ & -3.39595224 & 1.55548867 & -0.26951432 \\
\hline $\mathrm{C}$ & -3.38307586 & -1.26228377 & -0.27730407 \\
\hline $\mathrm{C}$ & -2.37683588 & -1.43585926 & -2.50518670 \\
\hline $\mathrm{C}$ & -1.77469778 & -2.45697692 & -1.51491617 \\
\hline $\mathrm{C}$ & -1.26132379 & -0.62604132 & -3.34621490 \\
\hline $\mathrm{C}$ & 2.56716659 & -1.02507433 & -1.49710616 \\
\hline $\mathrm{C}$ & 2.90110906 & 1.39081630 & -1.68203209 \\
\hline $\mathrm{F}$ & -3.20466832 & -2.04622044 & -3.42702508 \\
\hline $\mathrm{F}$ & -1.53959370 & -0.83958574 & -4.68234128 \\
\hline $\mathrm{F}$ & 3.33615446 & -3.64691122 & 1.33099635 \\
\hline
\end{tabular}




\begin{tabular}{|l|l|l|l|}
\hline $\mathrm{F}$ & 2.30376688 & -4.37894854 & -0.71902247 \\
\hline $\mathrm{F}$ & 2.63874239 & 3.67766795 & -2.39024531 \\
\hline $\mathrm{F}$ & 4.04374144 & 1.72054747 & -2.38483452 \\
\hline
\end{tabular}

\begin{tabular}{|c|c|c|c|}
\hline$' O '-\mathrm{C}_{60} \mathrm{~F}_{8}$ & & & \\
\hline $\mathrm{C}$ & 3.14058833 & 1.36773771 & 0.88245957 \\
\hline $\mathrm{C}$ & 2.89371506 & 0.64360276 & 2.11856295 \\
\hline $\mathrm{C}$ & 3.14174140 & -1.47339170 & 0.89605365 \\
\hline $\mathrm{C}$ & 2.88732227 & -0.74623307 & 2.11464649 \\
\hline $\mathrm{C}$ & 0.94987786 & 0.69055460 & 3.63209659 \\
\hline $\mathrm{C}$ & 1.88816614 & -1.47438567 & 2.88964862 \\
\hline $\mathrm{C}$ & 1.89558854 & 1.37681069 & 2.89549278 \\
\hline $\mathrm{C}$ & 0.94758311 & -0.77427881 & 3.63267846 \\
\hline $\mathrm{C}$ & 1.52257152 & -2.66409636 & 2.14716977 \\
\hline $\mathrm{C}$ & 1.50794795 & 2.55270795 & 2.11800826 \\
\hline $\bar{C}$ & -1.29888663 & -0.03666947 & 3.64314054 \\
\hline $\bar{C}$ & -0.44357828 & 1.14224470 & 3.63212010 \\
\hline $\bar{C}$ & 0.21632391 & -3.07008192 & 2.14238887 \\
\hline $\mathrm{C}$ & 2.52676620 & -2.88731649 & 0.99208294 \\
\hline $\mathrm{C}$ & -0.41316720 & -3.51875837 & 0.88468863 \\
\hline $\mathrm{C}$ & -0.79400159 & -2.34004549 & 2.90434387 \\
\hline $\mathrm{C}$ & -2.03724767 & -2.33248902 & 2.13491027 \\
\hline $\mathrm{C}$ & -0.43832746 & -1.22221265 & 3.64348521 \\
\hline $\mathrm{C}$ & -2.06182678 & 2.27392523 & 2.14572605 \\
\hline $\mathrm{C}$ & 0.18452825 & 2.97726153 & 2.11356205 \\
\hline $\mathrm{C}$ & -1.79165169 & -3.05806073 & 0.88848613 \\
\hline
\end{tabular}




\begin{tabular}{|c|c|c|c|}
\hline $\mathrm{C}$ & -2.84952666 & -1.21346152 & 2.13458501 \\
\hline $\mathrm{C}$ & -3.46295010 & -0.75560975 & 0.88781459 \\
\hline $\mathrm{C}$ & -2.47170926 & -0.02876239 & 2.90367050 \\
\hline $\mathrm{C}$ & -2.85256349 & 1.15775053 & 2.14115626 \\
\hline $\mathrm{C}$ & -0.42767959 & 3.44399715 & 0.89461715 \\
\hline $\mathrm{C}$ & 2.27327952 & 2.56258256 & 0.88211022 \\
\hline $\mathrm{C}$ & -0.81758995 & 2.25317998 & 2.88856003 \\
\hline $\mathrm{C}$ & -1.96259060 & 3.29742013 & 0.99027738 \\
\hline $\mathrm{C}$ & -3.47385361 & 0.69777730 & 0.88345573 \\
\hline $\mathrm{C}$ & 1.91694694 & -2.33888885 & -1.51647845 \\
\hline $\mathrm{C}$ & -2.37799833 & -2.62835134 & -0.29470754 \\
\hline $\mathrm{C}$ & 1.69146860 & 2.99347253 & -0.28013540 \\
\hline $\mathrm{C}$ & 2.35299110 & -0.04940550 & -2.27479665 \\
\hline $\mathrm{C}$ & -0.32432538 & -3.05761839 & -1.50020781 \\
\hline $\mathrm{C}$ & 3.34130431 & -0.76595804 & -0.26264897 \\
\hline $\mathrm{C}$ & 0.30114570 & -3.53045332 & -0.28383689 \\
\hline $\mathrm{C}$ & 0.68845555 & -2.31758344 & -2.26387332 \\
\hline $\mathrm{C}$ & 1.83417882 & -3.37866190 & -0.38135220 \\
\hline $\mathrm{C}$ & 3.37032258 & 0.68060866 & -0.27946100 \\
\hline $\mathrm{C}$ & 0.30709700 & 3.41409716 & -0.26386852 \\
\hline $\mathrm{C}$ & -0.31480741 & 2.95003768 & -1.51025288 \\
\hline $\mathrm{C}$ & -2.64396745 & 2.79062954 & -0.38315512 \\
\hline $\mathrm{C}$ & -1.62940570 & 2.54672248 & -1.51790054 \\
\hline $\mathrm{C}$ & 0.31087875 & 1.13246791 & -2.98144259 \\
\hline $\mathrm{C}$ & -1.98958177 & 1.37180012 & -2.26494972 \\
\hline $\mathrm{C}$ & 0.68272091 & 2.25163348 & -2.27547067 \\
\hline $\mathrm{C}$ & -1.05639341 & 0.68687275 & -3.00688068 \\
\hline
\end{tabular}




\begin{tabular}{|l|l|l|l|}
\hline C & -3.00722077 & 0.63845139 & -1.50129141 \\
\hline C & 2.08590301 & 2.51235968 & -1.69289711 \\
\hline C & 0.32658692 & -1.21838379 & -3.00632390 \\
\hline C & 1.17389082 & -0.05645290 & -2.98109540 \\
\hline C & -3.02378556 & -0.73307958 & -1.53000291 \\
\hline C & -3.26331484 & 1.38011426 & -0.28527038 \\
\hline C & -3.23573210 & -1.44669624 & -0.29505233 \\
\hline C & -2.23387085 & -1.62225362 & -2.51738171 \\
\hline C & -1.63348144 & -2.64841105 & -1.52943845 \\
\hline C & -1.12064042 & -0.81443060 & -3.35565790 \\
\hline C & 2.70773341 & -1.21394705 & -1.50903315 \\
\hline C & 3.03549276 & 1.20415837 & -1.69251428 \\
\hline F & -3.06783084 & -2.22788902 & -3.43674517 \\
\hline F & -1.40068160 & -1.01809821 & -4.69293922 \\
\hline F & 3.47622914 & -3.83518775 & 1.31996159 \\
\hline F & 2.44456916 & -4.56785325 & -0.72971816 \\
\hline F & 2.76030913 & 3.49076597 & -2.39679376 \\
\hline F & 4.17490730 & 1.54194499 & -2.39622187 \\
\hline F & -2.56971704 & 4.49397514 & 1.31753095 \\
\hline F & -3.58541275 & 3.73930890 & -0.73215489 \\
\hline & & & \\
\hline
\end{tabular}

\begin{tabular}{|l|l|l|l|}
\hline${ }^{\prime}-\mathrm{C}_{60} \mathrm{~F}_{10}$ & & & \\
\hline $\mathrm{C}$ & 3.02557433 & 1.28860065 & 0.69948190 \\
\hline $\mathrm{C}$ & 2.80780560 & 0.58387278 & 1.93448238 \\
\hline $\mathrm{C}$ & 3.03732689 & -1.52411207 & 0.70599638 \\
\hline $\mathrm{C}$ & 2.78954359 & -0.79087218 & 1.91845080 \\
\hline $\mathrm{C}$ & 0.92680812 & 0.67385510 & 3.78194142 \\
\hline
\end{tabular}




\begin{tabular}{|c|c|c|c|}
\hline $\mathrm{C}$ & 1.78552946 & -1.52458837 & 2.69933650 \\
\hline $\mathrm{C}$ & 2.03098127 & 1.47509843 & 2.92564691 \\
\hline $\mathrm{C}$ & 0.86432226 & -0.83393326 & 3.44607356 \\
\hline $\mathrm{C}$ & 1.42788943 & -2.71074324 & 1.95192550 \\
\hline $\mathrm{C}$ & 1.42459140 & 2.48945637 & 1.93391844 \\
\hline $\mathrm{C}$ & -1.36724262 & -0.08916970 & 3.42506814 \\
\hline $\mathrm{C}$ & -0.52604172 & 1.08150115 & 3.44551315 \\
\hline $\mathrm{C}$ & 0.12386080 & -3.12923738 & 1.94409102 \\
\hline $\mathrm{C}$ & 2.43205942 & -2.93880700 & 0.80176517 \\
\hline $\mathrm{C}$ & -0.50462876 & -3.58581301 & 0.68866250 \\
\hline $\mathrm{C}$ & -0.87901622 & -2.39615772 & 2.69915443 \\
\hline $\mathrm{C}$ & -2.12712438 & -2.39669149 & 1.93651026 \\
\hline $\mathrm{C}$ & -0.50937432 & -1.27101287 & 3.42541382 \\
\hline $\mathrm{C}$ & -2.13545510 & 2.19828553 & 1.95049079 \\
\hline $\mathrm{C}$ & 0.11184968 & 2.89804249 & 1.91736897 \\
\hline $\mathrm{C}$ & -1.88371349 & -3.12602626 & 0.69223403 \\
\hline $\mathrm{C}$ & -2.93836835 & -1.27908385 & 1.93618497 \\
\hline $\mathrm{C}$ & -3.55604438 & -0.82214709 & 0.69156113 \\
\hline $\mathrm{C}$ & -2.55134729 & -0.09227390 & 2.69848217 \\
\hline $\mathrm{C}$ & -2.93740408 & 1.08810064 & 1.94285989 \\
\hline $\mathrm{C}$ & -0.50819378 & 3.36035537 & 0.70456289 \\
\hline $\mathrm{C}$ & 2.16245928 & 2.47767400 & 0.69912908 \\
\hline $\mathrm{C}$ & -0.89706734 & 2.17108053 & 2.69825100 \\
\hline $\mathrm{C}$ & -2.04083345 & 3.22325766 & 0.79995991 \\
\hline $\mathrm{C}$ & -3.56589447 & 0.63152845 & 0.68742967 \\
\hline $\mathrm{C}$ & 1.82657724 & -2.40367329 & -1.71250209 \\
\hline $\mathrm{C}$ & -2.47014585 & -2.69543605 & -0.49128548 \\
\hline
\end{tabular}




\begin{tabular}{|c|c|c|c|}
\hline $\mathrm{C}$ & 1.59451872 & 2.91434231 & -0.47374350 \\
\hline $\mathrm{C}$ & 2.26278002 & -0.11529718 & -2.47525194 \\
\hline $\mathrm{C}$ & -0.41459275 & -3.12289360 & -1.69545775 \\
\hline $\mathrm{C}$ & 3.24322299 & -0.82546668 & -0.45969370 \\
\hline $\mathrm{C}$ & 0.21087070 & -3.59486285 & -0.47909767 \\
\hline $\mathrm{C}$ & 0.59769064 & -2.38317008 & -2.45982935 \\
\hline $\mathrm{C}$ & 1.74386728 & -3.43875373 & -0.57291966 \\
\hline $\mathrm{C}$ & 3.26511519 & 0.61286525 & -0.47306828 \\
\hline $\mathrm{C}$ & 0.22018660 & 3.33919623 & -0.46091387 \\
\hline $\mathrm{C}$ & -0.40516450 & 2.88490486 & -1.71006947 \\
\hline $\mathrm{C}$ & -2.72902877 & 2.72331348 & -0.57472481 \\
\hline $\mathrm{C}$ & -1.71894190 & 2.48079489 & -1.71393231 \\
\hline $\mathrm{C}$ & 0.22006012 & 1.06659974 & -3.18084625 \\
\hline $\mathrm{C}$ & -2.08000727 & 1.30574972 & -2.46091152 \\
\hline $\mathrm{C}$ & 0.59218425 & 2.18618723 & -2.47592366 \\
\hline $\mathrm{C}$ & -1.14747811 & 0.62094498 & -3.20383925 \\
\hline $\mathrm{C}$ & -3.09719528 & 0.57278148 & -1.69654205 \\
\hline $\mathrm{C}$ & 1.99477530 & 2.44358338 & -1.88730595 \\
\hline $\mathrm{C}$ & 0.23573723 & -1.28463624 & -3.20328082 \\
\hline $\mathrm{C}$ & 1.08318323 & -0.12247747 & -3.18049813 \\
\hline $\mathrm{C}$ & -3.11478156 & -0.79911011 & -1.72555493 \\
\hline $\mathrm{C}$ & -3.35247526 & 1.31416821 & -0.48053551 \\
\hline $\mathrm{C}$ & -3.32801804 & -1.51359001 & -0.49163073 \\
\hline $\mathrm{C}$ & -2.32478120 & -1.68829558 & -2.71316417 \\
\hline $\mathrm{C}$ & -1.72440990 & -2.71454575 & -1.72499230 \\
\hline $\mathrm{C}$ & -1.21181039 & -0.88066518 & -3.55192981 \\
\hline $\mathrm{C}$ & 2.61787211 & -1.27976404 & -1.70884912 \\
\hline
\end{tabular}




\begin{tabular}{|l|l|l|l|}
\hline C & 2.94193186 & 1.13874164 & -1.88692340 \\
\hline F & -3.15890285 & -2.29403744 & -3.63220620 \\
\hline F & -1.49240332 & -1.08473091 & -4.88898066 \\
\hline F & 3.38402345 & -3.88277064 & 1.13358729 \\
\hline F & 2.35910108 & -4.62734003 & -0.91374509 \\
\hline F & 2.66990652 & 3.42729298 & -2.58304451 \\
\hline F & 4.08660988 & 1.47558653 & -2.58247242 \\
\hline F & -2.64346541 & 4.42098774 & 1.13115386 \\
\hline F & -3.66840025 & 3.67641569 & -0.91618178 \\
\hline F & 1.21991338 & 0.88700245 & 5.11460589 \\
\hline F & 2.87388788 & 2.08721011 & 3.83279744 \\
\hline
\end{tabular}

\begin{tabular}{|l|l|l|l|}
\hline${ }^{\prime}{ }^{\prime} \mathrm{C}_{60} \mathrm{~F}_{12}$ & & & \\
\hline $\mathrm{C}$ & 3.17454446 & 1.39679873 & 0.58513915 \\
\hline $\mathrm{C}$ & 2.95617222 & 0.69140036 & 1.81993697 \\
\hline $\mathrm{C}$ & 3.18985716 & -1.41603321 & 0.59062155 \\
\hline $\mathrm{C}$ & 2.94155022 & -0.68285911 & 1.80338681 \\
\hline $\mathrm{C}$ & 1.06446080 & 0.77373498 & 3.65888147 \\
\hline $\mathrm{C}$ & 1.93040005 & -1.41660211 & 2.57642982 \\
\hline $\mathrm{C}$ & 2.17421479 & 1.57902716 & 2.81044924 \\
\hline $\mathrm{C}$ & 0.99839205 & -0.72926371 & 3.31670732 \\
\hline $\mathrm{C}$ & 1.56909871 & -2.59214929 & 1.82973540 \\
\hline $\mathrm{C}$ & 1.57283762 & 2.59715608 & 1.81937988 \\
\hline $\mathrm{C}$ & -1.23244318 & 0.01405183 & 3.29423487 \\
\hline $\mathrm{C}$ & -0.38494534 & 1.17649106 & 3.31615297 \\
\hline $\mathrm{C}$ & 0.25521215 & -2.99560390 & 1.82550251 \\
\hline
\end{tabular}




\begin{tabular}{|c|c|c|c|}
\hline $\mathrm{C}$ & 2.57771255 & -2.82902267 & 0.68648190 \\
\hline $\mathrm{C}$ & -0.37021725 & -3.44995067 & 0.57618806 \\
\hline $\mathrm{C}$ & -0.74172325 & -2.29512392 & 2.59063959 \\
\hline $\mathrm{C}$ & -2.14442217 & -2.55218666 & 2.00171809 \\
\hline $\mathrm{C}$ & -0.36942379 & -1.17488599 & 3.29458899 \\
\hline $\mathrm{C}$ & -1.97890199 & 2.29574963 & 1.82830578 \\
\hline $\mathrm{C}$ & 0.26168309 & 3.00905746 & 1.80230819 \\
\hline $\mathrm{C}$ & -1.74422687 & -3.02339992 & 0.58811386 \\
\hline $\mathrm{C}$ & -3.09158830 & -1.24732584 & 2.00133746 \\
\hline $\mathrm{C}$ & -3.41522090 & -0.72136217 & 0.58744245 \\
\hline $\mathrm{C}$ & -2.41270935 & 0.00691027 & 2.58996443 \\
\hline $\mathrm{C}$ & -2.76960331 & 1.17153446 & 1.82428520 \\
\hline $\mathrm{C}$ & -0.35813335 & 3.47185742 & 0.58919394 \\
\hline $\mathrm{C}$ & 2.31152568 & 2.58574295 & 0.58479220 \\
\hline $\mathrm{C}$ & -0.74946430 & 2.27531849 & 2.57535552 \\
\hline $\mathrm{C}$ & -1.89128278 & 3.32768418 & 0.68468267 \\
\hline $\mathrm{C}$ & -3.39503423 & 0.71718501 & 0.57497133 \\
\hline $\mathrm{C}$ & 1.97889429 & -2.29574947 & -1.82830352 \\
\hline $\mathrm{C}$ & -2.31152146 & -2.58573571 & -0.58479231 \\
\hline $\mathrm{C}$ & 1.74423196 & 3.02340109 & -0.58811562 \\
\hline $\mathrm{C}$ & 2.41271249 & -0.00691127 & -2.58997072 \\
\hline $\mathrm{C}$ & -0.26168263 & -3.00905996 & -1.80230554 \\
\hline $\mathrm{C}$ & 3.39503039 & -0.71718450 & -0.57496514 \\
\hline $\mathrm{C}$ & 0.35813471 & -3.47186045 & -0.58919047 \\
\hline $\mathrm{C}$ & 0.74946458 & -2.27532095 & -2.57535667 \\
\hline $\mathrm{C}$ & 1.89128037 & -3.32768762 & -0.68468138 \\
\hline $\mathrm{C}$ & 3.41521645 & 0.72136781 & -0.58744260 \\
\hline
\end{tabular}




\begin{tabular}{|c|c|c|c|}
\hline $\mathrm{C}$ & 0.37021855 & 3.44994931 & -0.57618326 \\
\hline $\mathrm{C}$ & -0.25521569 & 2.99559798 & -1.82550121 \\
\hline $\mathrm{C}$ & -2.57771405 & 2.82902165 & -0.68647911 \\
\hline $\mathrm{C}$ & -1.56909754 & 2.59214319 & -1.82973500 \\
\hline $\mathrm{C}$ & 0.36942705 & 1.17488701 & -3.29459288 \\
\hline $\mathrm{C}$ & -1.93040297 & 1.41659794 & -2.57643360 \\
\hline $\mathrm{C}$ & 0.74172668 & 2.29512192 & -2.59064251 \\
\hline $\mathrm{C}$ & -0.99839272 & 0.72926393 & -3.31670977 \\
\hline $\mathrm{C}$ & -2.94155178 & 0.68286093 & -1.80338389 \\
\hline $\mathrm{C}$ & 2.14441447 & 2.55220550 & -2.00172198 \\
\hline $\mathrm{C}$ & 0.38494356 & -1.17649003 & -3.31614860 \\
\hline $\mathrm{C}$ & 1.23244904 & -0.01405466 & -3.29424594 \\
\hline $\mathrm{C}$ & -2.95617288 & -0.69139960 & -1.81993782 \\
\hline $\mathrm{C}$ & -3.18986149 & 1.41603671 & -0.59061817 \\
\hline $\mathrm{C}$ & -3.17454093 & -1.39679675 & -0.58514004 \\
\hline $\mathrm{C}$ & -2.17421430 & -1.57902609 & -2.81044797 \\
\hline $\mathrm{C}$ & -1.57283690 & -2.59715535 & -1.81938348 \\
\hline $\mathrm{C}$ & -1.06446176 & -0.77373619 & -3.65888128 \\
\hline $\mathrm{C}$ & 2.76959715 & -1.17153723 & -1.82428265 \\
\hline $\mathrm{C}$ & 3.09160095 & 1.24731364 & -2.00134054 \\
\hline $\mathrm{F}$ & -3.01190471 & -2.18734564 & -3.72455902 \\
\hline $\mathrm{F}$ & -1.35241649 & -0.98314998 & -4.99327536 \\
\hline $\mathrm{F}$ & 3.52435527 & -3.77543557 & 1.02639839 \\
\hline $\mathrm{F}$ & 2.49789474 & -4.52111576 & -1.02397310 \\
\hline $\mathrm{F}$ & 2.81974034 & 3.53568836 & -2.69747804 \\
\hline $\mathrm{F}$ & 4.23612683 & 1.58440602 & -2.69690509 \\
\hline $\mathrm{F}$ & -2.49789984 & 4.52111159 & 1.02397434 \\
\hline
\end{tabular}




\begin{tabular}{|l|l|l|l|}
\hline $\mathrm{F}$ & -3.52435720 & 3.77543490 & -1.02639369 \\
\hline $\mathrm{F}$ & 1.35241702 & 0.98315088 & 4.99327521 \\
\hline $\mathrm{F}$ & 3.01190605 & 2.18734778 & 3.72456061 \\
\hline $\mathrm{F}$ & -2.81973300 & -3.53569257 & 2.69747470 \\
\hline $\mathrm{F}$ & -4.23612685 & -1.58439580 & 2.69690506 \\
\hline
\end{tabular}

\begin{tabular}{|l|l|l|l|}
\hline${ }^{\prime}{ }^{\prime}-\mathrm{C}_{60} \mathrm{~F}_{6}$ & & & \\
\hline $\mathrm{C}$ & 3.46062144 & 1.24222454 & 1.11561460 \\
\hline $\mathrm{C}$ & 3.22814824 & 0.50771471 & 2.35976459 \\
\hline $\mathrm{C}$ & 3.46575902 & -1.61030507 & 1.11071993 \\
\hline $\mathrm{C}$ & 3.22800092 & -0.87850851 & 2.35577676 \\
\hline $\mathrm{C}$ & 1.26700361 & 0.54406122 & 3.85374230 \\
\hline $\mathrm{C}$ & 2.22096034 & -1.61196448 & 3.12388225 \\
\hline $\mathrm{C}$ & 2.22170218 & 1.23916533 & 3.13135553 \\
\hline $\mathrm{C}$ & 1.26726392 & -0.91960909 & 3.85202485 \\
\hline $\mathrm{C}$ & 1.83791811 & -2.79745352 & 2.35561916 \\
\hline $\mathrm{C}$ & 1.83767462 & 2.42560552 & 2.36240756 \\
\hline $\mathrm{C}$ & -0.98454584 & -0.19043130 & 3.85131985 \\
\hline $\mathrm{C}$ & -0.12581260 & 0.99675785 & 3.84823791 \\
\hline $\mathrm{C}$ & 0.52331472 & -3.23135690 & 2.35825185 \\
\hline $\mathrm{C}$ & 2.60375653 & -2.79512172 & 1.10726516 \\
\hline $\mathrm{C}$ & -0.09982956 & -3.68684341 & 1.11301248 \\
\hline $\mathrm{C}$ & -0.48521842 & -2.49839157 & 3.12566873 \\
\hline $\mathrm{C}$ & -1.72697768 & -2.50041363 & 2.35140345 \\
\hline $\mathrm{C}$ & -0.12390191 & -1.37428638 & 3.85193874 \\
\hline & -1.71912650 & 2.10990364 & 2.33161446 \\
\hline & & & \\
\hline & & & \\
\hline & & & \\
\hline & & & \\
\hline & & & \\
\hline & & & \\
\hline & & & \\
\hline & & & \\
\hline & & & \\
\hline & & & \\
\hline
\end{tabular}




\begin{tabular}{|c|c|c|c|}
\hline $\mathrm{C}$ & 0.51956987 & 2.85387922 & 2.35051315 \\
\hline $\mathrm{C}$ & -1.48323868 & -3.22938478 & 1.10389891 \\
\hline $\mathrm{C}$ & -2.53785286 & -1.37960230 & 2.34907109 \\
\hline $\mathrm{C}$ & -3.12148993 & -0.92145618 & 1.08745566 \\
\hline $\mathrm{C}$ & -2.16151486 & -0.19389557 & 3.12194235 \\
\hline $\mathrm{C}$ & -2.53054357 & 0.98801990 & 2.33918118 \\
\hline $\mathrm{C}$ & -0.09231946 & 3.31951775 & 1.10707629 \\
\hline $\mathrm{C}$ & 2.59364707 & 2.42350754 & 1.11674529 \\
\hline $\mathrm{C}$ & -0.48531671 & 2.11565613 & 3.11514826 \\
\hline $\mathrm{C}$ & -1.48561115 & 2.83707198 & 1.08706514 \\
\hline $\mathrm{C}$ & -3.10140722 & 0.53116164 & 1.08153927 \\
\hline $\mathrm{C}$ & 2.23033162 & -2.48090976 & -1.30928444 \\
\hline $\mathrm{C}$ & -2.04869964 & -2.79515522 & -0.08805502 \\
\hline $\mathrm{C}$ & 1.97768465 & 2.84669390 & -0.05396366 \\
\hline $\mathrm{C}$ & 2.62223062 & -0.18339986 & -2.03981777 \\
\hline $\mathrm{C}$ & -0.01172283 & -3.24401029 & -1.31514516 \\
\hline $\mathrm{C}$ & 3.68975867 & -0.91522480 & -0.06569309 \\
\hline $\mathrm{C}$ & 0.62012687 & -3.68101219 & -0.07129699 \\
\hline $\mathrm{C}$ & 0.98954603 & -2.47705324 & -2.07911769 \\
\hline $\mathrm{C}$ & 2.00865893 & -3.22121925 & -0.06870116 \\
\hline $\mathrm{C}$ & 3.68194151 & 0.54928165 & -0.06107104 \\
\hline $\mathrm{C}$ & 0.61634036 & 3.34368887 & -0.06153442 \\
\hline $\mathrm{C}$ & 0.01049326 & 3.18077019 & -1.48283861 \\
\hline $\mathrm{C}$ & -2.03150490 & 2.40807650 & -0.09491256 \\
\hline $\mathrm{C}$ & -1.52363059 & 2.73125900 & -1.49428201 \\
\hline $\mathrm{C}$ & 0.56045127 & 0.99026970 & -2.72818349 \\
\hline $\mathrm{C}$ & -1.95626377 & 1.38559770 & -2.37313880 \\
\hline
\end{tabular}




\begin{tabular}{|c|c|c|c|}
\hline $\mathrm{C}$ & 0.93854441 & 2.09792887 & -2.03937164 \\
\hline $\mathrm{C}$ & -0.83332809 & 0.63894770 & -3.20561272 \\
\hline $\mathrm{C}$ & -2.59232960 & 0.48436439 & -1.33526294 \\
\hline $\mathrm{C}$ & 2.17925563 & 2.09150719 & -1.28111730 \\
\hline $\mathrm{C}$ & 0.59505573 & -1.36557635 & -2.77776348 \\
\hline $\mathrm{C}$ & 1.40494161 & -0.18112332 & -2.70889856 \\
\hline $\mathrm{C}$ & -2.62597670 & -0.87327756 & -1.33523119 \\
\hline $\mathrm{C}$ & -2.80954556 & 1.20051239 & -0.10009210 \\
\hline $\mathrm{C}$ & -2.86715077 & -1.59226721 & -0.09456816 \\
\hline $\mathrm{C}$ & -1.91541788 & -1.79827521 & -2.32671515 \\
\hline $\mathrm{C}$ & -1.31607437 & -2.83585946 & -1.33782407 \\
\hline $\mathrm{C}$ & -0.82673714 & -1.02548489 & -3.20608711 \\
\hline $\mathrm{C}$ & 3.04823415 & -1.36373303 & -1.30388035 \\
\hline $\mathrm{C}$ & 3.02087604 & 0.99447717 & -1.28866957 \\
\hline $\mathrm{F}$ & -2.79816294 & -2.39797802 & -3.20499632 \\
\hline $\mathrm{F}$ & -1.05430090 & -1.39310200 & -4.51948396 \\
\hline $\mathrm{F}$ & 0.09558934 & 4.35936498 & -2.19973044 \\
\hline $\mathrm{F}$ & -2.23248112 & 3.77847999 & -2.05356594 \\
\hline $\mathrm{F}$ & -2.89453103 & 1.83513585 & -3.28668764 \\
\hline $\mathrm{F}$ & -1.01392880 & 1.00142178 & -4.52962003 \\
\hline
\end{tabular}

\begin{tabular}{|l|l|l|l|}
\hline $\mathrm{S}^{\prime}-\mathrm{C}_{60} \mathrm{~F}_{8}$ & & & \\
\hline $\mathrm{C}$ & 3.50594612 & 1.43886747 & 1.20768894 \\
\hline $\mathrm{C}$ & 3.27691047 & 0.70613062 & 2.45394051 \\
\hline $\mathrm{C}$ & 3.51415585 & -1.41539731 & 1.21154768 \\
\hline $\mathrm{C}$ & 3.28089907 & -0.68062442 & 2.45581662 \\
\hline $\mathrm{C}$ & 1.31546044 & 0.74083345 & 3.94684329 \\
\hline
\end{tabular}




\begin{tabular}{|c|c|c|c|}
\hline $\mathrm{C}$ & 2.27721452 & -1.41539808 & 3.22735892 \\
\hline $\mathrm{C}$ & 2.26900994 & 1.43719931 & 3.22350364 \\
\hline $\mathrm{C}$ & 1.31967044 & -0.72258047 & 3.94882228 \\
\hline $\mathrm{C}$ & 1.89786219 & -2.60439968 & 2.46003461 \\
\hline $\mathrm{C}$ & 1.88282954 & 2.62192459 & 2.45297453 \\
\hline $\mathrm{C}$ & -0.93392045 & 0.00264129 & 3.94300492 \\
\hline $\mathrm{C}$ & -0.07787328 & 1.19133269 & 3.93946870 \\
\hline $\mathrm{C}$ & 0.58208782 & -3.03956651 & 2.44923613 \\
\hline $\mathrm{C}$ & 2.65290139 & -2.60085396 & 1.21363942 \\
\hline $\mathrm{C}$ & -0.02776315 & -3.51344240 & 1.20734124 \\
\hline $\mathrm{C}$ & -0.42576549 & -2.30393958 & 3.21195450 \\
\hline $\mathrm{C}$ & -1.65966032 & -2.30699337 & 2.42845747 \\
\hline $\mathrm{C}$ & -0.07104861 & -1.18110696 & 3.94267307 \\
\hline $\mathrm{C}$ & -1.67292570 & 2.30396367 & 2.42223298 \\
\hline $\mathrm{C}$ & 0.56456986 & 3.04947764 & 2.44101010 \\
\hline $\mathrm{C}$ & -1.42351271 & -3.03790727 & 1.18580682 \\
\hline $\mathrm{C}$ & -2.47409610 & -1.18715181 & 2.43332514 \\
\hline $\mathrm{C}$ & -3.03413108 & -0.73271027 & 1.16962571 \\
\hline $\mathrm{C}$ & -2.11029034 & -0.00172902 & 3.21408281 \\
\hline $\mathrm{C}$ & -2.48090897 & 1.17946964 & 2.43013231 \\
\hline $\mathrm{C}$ & -0.04799320 & 3.51647954 & 1.19784334 \\
\hline $\mathrm{C}$ & 2.63788575 & 2.61935117 & 1.20658489 \\
\hline $\mathrm{C}$ & -0.43903845 & 2.31012541 & 3.20572108 \\
\hline $\mathrm{C}$ & -1.44097902 & 3.03286410 & 1.17760746 \\
\hline $\mathrm{C}$ & -3.03830751 & 0.71839397 & 1.16766685 \\
\hline $\mathrm{C}$ & 2.23100595 & -2.27536951 & -1.18371021 \\
\hline $\mathrm{C}$ & -1.96902462 & -2.61573290 & 0.00059444 \\
\hline
\end{tabular}




\begin{tabular}{|c|c|c|c|}
\hline $\mathrm{C}$ & 2.02056246 & 3.04248291 & 0.03609328 \\
\hline $\mathrm{C}$ & 2.64113225 & 0.00499419 & -1.92864157 \\
\hline $\mathrm{C}$ & 0.07292390 & -3.38354055 & -1.38492902 \\
\hline $\mathrm{C}$ & 3.72847929 & -0.72155165 & 0.03383093 \\
\hline $\mathrm{C}$ & 0.67991419 & -3.53759109 & 0.03812760 \\
\hline $\mathrm{C}$ & 0.98965692 & -2.29175885 & -1.94187222 \\
\hline $\mathrm{C}$ & 2.03803829 & -3.03069264 & 0.04429919 \\
\hline $\mathrm{C}$ & 3.72426721 & 0.74308828 & 0.03185072 \\
\hline $\mathrm{C}$ & 0.65954125 & 3.54154253 & 0.02856711 \\
\hline $\mathrm{C}$ & 0.05345479 & 3.38013677 & -1.39406603 \\
\hline $\mathrm{C}$ & -1.98404871 & 2.60435146 & -0.00645163 \\
\hline $\mathrm{C}$ & -1.48031987 & 2.93043275 & -1.40688370 \\
\hline $\mathrm{C}$ & 0.58894572 & 1.17977631 & -2.62598944 \\
\hline $\mathrm{C}$ & -1.91342248 & 1.58149343 & -2.28727934 \\
\hline $\mathrm{C}$ & 0.97646824 & 2.29215138 & -1.94806697 \\
\hline $\mathrm{C}$ & -0.79779786 & 0.83166665 & -3.12068378 \\
\hline $\mathrm{C}$ & -2.51970158 & 0.67092199 & -1.24352705 \\
\hline $\mathrm{C}$ & 2.21788137 & 2.28496125 & -1.18987487 \\
\hline $\mathrm{C}$ & 0.59575040 & -1.18346501 & -2.62279011 \\
\hline $\mathrm{C}$ & 1.41574400 & 0.00059338 & -2.57528592 \\
\hline $\mathrm{C}$ & -2.51579802 & -0.68876516 & -1.24168904 \\
\hline $\mathrm{C}$ & -2.74795629 & 1.38833077 & -0.01214633 \\
\hline $\mathrm{C}$ & -2.73992504 & -1.40414924 & -0.00837520 \\
\hline $\mathrm{C}$ & -1.90427371 & -1.59863518 & -2.28298590 \\
\hline $\mathrm{C}$ & -1.46341916 & -2.94270638 & -1.39894565 \\
\hline $\mathrm{C}$ & -0.79297310 & -0.84468310 & -3.11841407 \\
\hline $\mathrm{C}$ & 3.06414963 & -1.17171681 & -1.19052142 \\
\hline
\end{tabular}




\begin{tabular}{|l|l|l|l|}
\hline C & 3.05736780 & 1.18610859 & -1.19370744 \\
\hline$F$ & -2.85166382 & -2.05156871 & -3.18369854 \\
\hline$F$ & -0.96219606 & -1.20679041 & -4.44196425 \\
\hline$F$ & 0.14117528 & 4.55821584 & -2.11062186 \\
\hline$F$ & -2.19119369 & 3.97595008 & -1.96503268 \\
\hline$F$ & -2.86339856 & 2.02651053 & -3.18922012 \\
\hline$F$ & -0.96910044 & 1.18920665 & -4.44520933 \\
\hline$F$ & 0.16742876 & -4.56302257 & -2.09829736 \\
\hline$F$ & -2.16825966 & -3.99378674 & -1.95427072 \\
\hline
\end{tabular}

\begin{tabular}{|l|l|l|l|}
\hline${ }^{\prime}{ }^{\prime}-\mathrm{C}_{60} \mathrm{~F}_{10}$ & & & \\
\hline $\mathrm{C}$ & 1.582788 & -3.299975 & -1.819482 \\
\hline $\mathrm{C}$ & 2.353585 & -3.305145 & -0.576918 \\
\hline $\mathrm{C}$ & 1.7545 & -3.741025 & 0.594803 \\
\hline $\mathrm{C}$ & 0.361016 & -4.192263 & 0.589707 \\
\hline $\mathrm{C}$ & -0.363708 & -4.192059 & -0.589518 \\
\hline $\mathrm{C}$ & 0.2656 & -3.732171 & -1.829468 \\
\hline $\mathrm{C}$ & 1.96978 & -2.10679 & -2.570561 \\
\hline $\mathrm{C}$ & 2.974717 & -1.374281 & -1.804054 \\
\hline $\mathrm{C}$ & 3.229736 & -2.134276 & -0.566329 \\
\hline $\mathrm{C}$ & 3.477823 & -1.448084 & 0.589762 \\
\hline $\mathrm{C}$ & 1.98915 & -2.99958 & 1.827055 \\
\hline $\mathrm{C}$ & -0.268013 & -3.731932 & 1.829627 \\
\hline $\mathrm{C}$ & -1.584935 & -3.298936 & 1.819631 \\
\hline $\mathrm{C}$ & -2.355727 & -3.303745 & 0.577079 \\
\hline $\mathrm{C}$ & -1.756919 & -3.740001 & -0.594631 \\
\hline & & & \\
\hline
\end{tabular}




\begin{tabular}{|c|c|c|c|}
\hline $\mathrm{C}$ & -1.991121 & -2.998484 & -1.82694 \\
\hline $\mathrm{C}$ & -0.744681 & -2.999233 & -2.596424 \\
\hline $\mathrm{C}$ & -0.379651 & -1.875181 & -3.314556 \\
\hline $\mathrm{C}$ & 1.012608 & -1.418606 & -3.297483 \\
\hline $\mathrm{C}$ & 2.975762 & -0.00301 & -1.760067 \\
\hline $\mathrm{C}$ & 1.930021 & 0.706938 & -2.440767 \\
\hline $\mathrm{C}$ & 1.005026 & 0.034936 & -3.22692 \\
\hline $\mathrm{C}$ & -0.375389 & 0.479543 & -3.206176 \\
\hline $\mathrm{C}$ & -1.236318 & -0.688397 & -3.268499 \\
\hline $\mathrm{C}$ & -2.400789 & -0.677778 & -2.522626 \\
\hline $\mathrm{C}$ & -2.807243 & -1.873541 & -1.800824 \\
\hline $\mathrm{C}$ & -3.478864 & -1.446119 & -0.589738 \\
\hline $\mathrm{C}$ & -3.231133 & -2.132328 & 0.566428 \\
\hline $\mathrm{C}$ & 0.742712 & -2.999565 & 2.596566 \\
\hline $\mathrm{C}$ & -1.929678 & 0.708316 & 2.440598 \\
\hline $\mathrm{C}$ & -1.005107 & 0.035756 & 3.226876 \\
\hline $\mathrm{C}$ & 0.375574 & 0.479498 & 3.206249 \\
\hline $\mathrm{C}$ & 0.728593 & 1.548419 & 2.401217 \\
\hline $\mathrm{C}$ & -0.264027 & 2.299536 & 1.676967 \\
\hline $\mathrm{C}$ & -2.632961 & 2.231234 & 0.68238 \\
\hline $\mathrm{C}$ & -3.605722 & 0.873716 & 0.683373 \\
\hline $\mathrm{C}$ & -2.97586 & -0.001107 & 1.760027 \\
\hline $\mathrm{C}$ & -2.975675 & -1.372426 & 1.804107 \\
\hline $\mathrm{C}$ & -1.971188 & -2.105473 & 2.570634 \\
\hline $\mathrm{C}$ & -1.013614 & -1.417779 & 3.297506 \\
\hline $\mathrm{C}$ & 1.235798 & -0.689012 & 3.268611 \\
\hline $\mathrm{C}$ & 2.400293 & -0.679095 & 2.522753 \\
\hline
\end{tabular}




\begin{tabular}{|c|c|c|c|}
\hline $\mathrm{C}$ & 2.798126 & 0.489868 & 1.752462 \\
\hline $\mathrm{C}$ & 1.98722 & 1.579863 & 1.701263 \\
\hline $\mathrm{C}$ & 1.995 & 2.701007 & 0.685026 \\
\hline $\mathrm{C}$ & 0.398213 & 3.223355 & 0.682222 \\
\hline $\mathrm{C}$ & -0.395885 & 3.223607 & -0.682242 \\
\hline $\mathrm{C}$ & -1.993303 & 2.702156 & -0.684886 \\
\hline $\mathrm{C}$ & -3.80962 & 0.070649 & -0.683934 \\
\hline $\mathrm{C}$ & -2.797767 & 0.491415 & -1.752285 \\
\hline $\mathrm{C}$ & -1.986122 & 1.580888 & -1.701078 \\
\hline $\mathrm{C}$ & -0.727565 & 1.548721 & -2.401086 \\
\hline $\mathrm{C}$ & 0.265641 & 2.299267 & -1.676955 \\
\hline $\mathrm{C}$ & 1.560855 & 1.882776 & -1.688376 \\
\hline $\mathrm{C}$ & 2.635082 & 2.228807 & -0.682632 \\
\hline $\mathrm{C}$ & 3.605838 & 0.872038 & -0.683486 \\
\hline $\mathrm{C}$ & 3.809474 & 0.068472 & 0.683959 \\
\hline $\mathrm{C}$ & 0.378366 & -1.875257 & 3.314666 \\
\hline $\mathrm{C}$ & 2.805935 & -1.87509 & 1.800862 \\
\hline $\mathrm{C}$ & -1.559557 & 1.883898 & 1.688347 \\
\hline $\mathrm{F}$ & -3.443361 & 3.258675 & 1.129561 \\
\hline $\mathrm{F}$ & -2.724548 & 3.787599 & -1.128146 \\
\hline $\bar{F}$ & -0.442051 & 4.527036 & -1.137404 \\
\hline $\mathrm{F}$ & 0.445018 & 4.526824 & 1.137365 \\
\hline $\mathrm{F}$ & -4.855796 & 1.320533 & 1.066633 \\
\hline $\mathrm{F}$ & -5.11541 & 0.27237 & -1.087305 \\
\hline $\mathrm{F}$ & 2.727334 & 3.785943 & 1.127455 \\
\hline $\mathrm{F}$ & 3.445418 & 3.256583 & -1.129484 \\
\hline $\mathrm{F}$ & 4.856791 & 1.316963 & -1.066677 \\
\hline
\end{tabular}




\begin{tabular}{|l|l|l|l|}
\hline $\mathrm{F}$ & 5.115477 & 0.269519 & 1.086786 \\
\hline
\end{tabular}

\begin{tabular}{|c|c|c|c|}
\hline$' \mathrm{~S}^{\prime}-\mathrm{C}_{60} \mathrm{~F}_{12}$ & & & \\
\hline $\mathrm{C}$ & -0.692719 & -3.573126 & 1.864145 \\
\hline $\mathrm{C}$ & -1.431852 & -3.837626 & 0.629652 \\
\hline $\bar{C}$ & -0.73274 & -4.088779 & -0.540166 \\
\hline $\mathrm{C}$ & 0.733493 & -4.088696 & -0.540139 \\
\hline $\mathrm{C}$ & 1.432525 & -3.837474 & 0.629713 \\
\hline $\mathrm{C}$ & 0.693304 & -3.573042 & 1.864174 \\
\hline $\mathrm{C}$ & -1.430018 & -2.548542 & 2.603962 \\
\hline $\mathrm{C}$ & -2.614792 & -2.173738 & 1.836049 \\
\hline $\mathrm{C}$ & -2.625808 & -2.993707 & 0.610625 \\
\hline $\mathrm{C}$ & -3.07715 & -2.433711 & -0.552414 \\
\hline $\mathrm{C}$ & -1.186426 & -3.47007 & -1.779089 \\
\hline $\mathrm{C}$ & 1.187146 & -3.469907 & -1.779053 \\
\hline $\mathrm{C}$ & 2.309468 & -2.64891 & -1.761833 \\
\hline $\mathrm{C}$ & 3.077704 & -2.433389 & -0.55233 \\
\hline $\bar{C}$ & 2.626365 & -2.993371 & 0.610718 \\
\hline $\mathrm{C}$ & 2.61522 & -2.173399 & 1.836159 \\
\hline $\mathrm{C}$ & 1.430457 & -2.548331 & 2.603982 \\
\hline $\mathrm{C}$ & 0.733337 & -1.587318 & 3.315494 \\
\hline $\mathrm{C}$ & -0.733033 & -1.587466 & 3.315541 \\
\hline $\bar{C}$ & -3.041518 & -0.870695 & 1.775358 \\
\hline $\mathrm{C}$ & -2.267061 & 0.138851 & 2.440191 \\
\hline $\mathrm{C}$ & -1.176627 & -0.203866 & 3.22695 \\
\hline $\bar{C}$ & -0.000025 & 0.643322 & 3.177828 \\
\hline
\end{tabular}




\begin{tabular}{|c|c|c|c|}
\hline $\mathrm{C}$ & 1.176683 & -0.203657 & 3.226804 \\
\hline $\mathrm{C}$ & 2.267061 & 0.139229 & 2.43994 \\
\hline $\mathrm{C}$ & 3.041734 & -0.870254 & 1.77534 \\
\hline $\mathrm{C}$ & 3.915571 & -0.249508 & 0.692619 \\
\hline $\bar{C}$ & 3.862076 & -1.094379 & -0.663123 \\
\hline $\mathrm{C}$ & 0.000351 & -3.094004 & -2.551194 \\
\hline $\mathrm{C}$ & 1.391621 & 1.245119 & -2.410327 \\
\hline $\mathrm{C}$ & 0.724974 & 0.324624 & -3.200012 \\
\hline $\mathrm{C}$ & -0.724836 & 0.324508 & -3.20011 \\
\hline $\mathrm{C}$ & -1.39174 & 1.244863 & -2.410514 \\
\hline $\mathrm{C}$ & -0.680982 & 2.278684 & -1.702102 \\
\hline $\mathrm{C}$ & 1.598997 & 2.969088 & -0.72209 \\
\hline $\mathrm{C}$ & 2.955025 & 1.972989 & -0.707831 \\
\hline $\mathrm{C}$ & 2.598782 & 0.89438 & -1.707715 \\
\hline $\mathrm{C}$ & 3.031259 & -0.394726 & -1.741438 \\
\hline $\mathrm{C}$ & 2.29302 & -1.39431 & -2.4987 \\
\hline $\mathrm{C}$ & 1.181524 & -1.053439 & -3.248123 \\
\hline $\mathrm{C}$ & -1.181132 & -1.053656 & -3.248261 \\
\hline $\mathrm{C}$ & -2.292625 & -1.39468 & -2.498912 \\
\hline $\mathrm{C}$ & -3.031131 & -0.395199 & -1.741776 \\
\hline $\mathrm{C}$ & -2.598931 & 0.893977 & -1.707997 \\
\hline $\mathrm{C}$ & -2.955242 & 1.972484 & -0.708125 \\
\hline $\mathrm{C}$ & -1.599711 & 2.968633 & -0.722176 \\
\hline $\mathrm{C}$ & -0.84271 & 3.235429 & 0.637057 \\
\hline $\mathrm{C}$ & 0.841924 & 3.235619 & 0.637084 \\
\hline $\mathrm{C}$ & 3.414445 & 1.344647 & 0.668929 \\
\hline $\mathrm{C}$ & 2.283588 & 1.362412 & 1.671522 \\
\hline
\end{tabular}




\begin{tabular}{|l|l|l|l|}
\hline C & 1.181855 & 2.161068 & 1.643246 \\
\hline C & -0.000232 & 1.756678 & 2.35855 \\
\hline C & -1.182422 & 2.160821 & 1.643228 \\
\hline C & -2.283953 & 1.361967 & 1.671577 \\
\hline C & -3.415113 & 1.343127 & 0.669089 \\
\hline C & -3.915308 & -0.249323 & 0.692669 \\
\hline C & -3.861693 & -1.094956 & -0.663371 \\
\hline C & 0.000273 & -1.918455 & -3.277689 \\
\hline C & -2.308834 & -2.649217 & -1.761907 \\
\hline C & 0.680565 & 2.278852 & -1.702068 \\
\hline F & 2.050517 & 4.184729 & -1.19636 \\
\hline F & 1.200703 & 4.493749 & 1.078063 \\
\hline F & -1.201802 & 4.493483 & 1.078023 \\
\hline F & -2.051299 & 4.184259 & -1.196555 \\
\hline F & 3.988656 & 2.769246 & -1.161819 \\
\hline F & 4.504028 & 2.073695 & 1.108073 \\
\hline F & -3.989374 & 2.768399 & -1.161336 \\
\hline F & -4.504429 & -0.21025 & 1.0787634 \\
\hline F & -5.241526 & -1.312565 & -1.062403 \\
\hline F & -5.166077 & & \\
\hline F & 5.241542 & \\
\hline F & & & \\
\hline & & & \\
\hline & & & \\
\hline
\end{tabular}

\begin{tabular}{|l|l|l|l|}
\hline $\mathrm{S}^{\prime}-\mathrm{C}_{60} \mathrm{~F}_{14}$ & & & \\
\hline $\mathrm{C}$ & 0.243737 & -3.55446 & 1.81275 \\
\hline $\mathrm{C}$ & -0.376966 & -4.023367 & 0.582099 \\
\hline $\mathrm{C}$ & 0.377083 & -4.023389 & -0.58211 \\
\hline
\end{tabular}




\begin{tabular}{|c|c|c|c|}
\hline $\mathrm{C}$ & 1.774828 & -3.58778 & -0.564616 \\
\hline $\mathrm{C}$ & 2.370877 & -3.175728 & 0.594042 \\
\hline $\mathrm{C}$ & 1.565948 & -3.124874 & 1.798884 \\
\hline $\mathrm{C}$ & -0.772172 & -2.815559 & 2.562665 \\
\hline $\mathrm{C}$ & -2.017075 & -2.816539 & 1.798243 \\
\hline $\mathrm{C}$ & -1.774736 & -3.587844 & 0.564633 \\
\hline $\mathrm{C}$ & -2.370818 & -3.175862 & -0.594004 \\
\hline $\mathrm{C}$ & -0.243632 & -3.554501 & -1.812732 \\
\hline $\mathrm{C}$ & 2.017171 & -2.816504 & -1.798239 \\
\hline $\mathrm{C}$ & 2.822776 & -1.705893 & -1.750077 \\
\hline $\mathrm{C}$ & 3.844756 & -1.371349 & -0.670684 \\
\hline $\mathrm{C}$ & 3.529544 & -2.142088 & 0.694752 \\
\hline $\mathrm{C}$ & 2.953377 & -1.205545 & 1.759075 \\
\hline $\mathrm{C}$ & 1.939774 & -1.917918 & 2.522288 \\
\hline $\mathrm{C}$ & 0.98928 & -1.239276 & 3.261816 \\
\hline $\mathrm{C}$ & -0.404097 & -1.69249 & 3.281185 \\
\hline $\mathrm{C}$ & -2.822715 & -1.705955 & 1.750089 \\
\hline $\mathrm{C}$ & -2.39758 & -0.513725 & 2.427986 \\
\hline $\mathrm{C}$ & -1.253092 & -0.512054 & 3.213183 \\
\hline $\mathrm{C}$ & -0.396644 & 0.658489 & 3.174169 \\
\hline $\mathrm{C}$ & 0.982903 & 0.212131 & 3.195832 \\
\hline $\mathrm{C}$ & 1.903959 & 0.872124 & 2.401055 \\
\hline $\mathrm{C}$ & 2.943058 & 0.154116 & 1.709173 \\
\hline $\mathrm{C}$ & 3.618769 & 1.056601 & 0.69961 \\
\hline $\mathrm{C}$ & 3.861593 & 0.298762 & -0.668062 \\
\hline $\mathrm{C}$ & 0.772265 & -2.815569 & -2.562626 \\
\hline $\mathrm{C}$ & 0.741725 & 1.729756 & -2.371678 \\
\hline
\end{tabular}




\begin{tabular}{|c|c|c|c|}
\hline $\mathrm{C}$ & 0.396629 & 0.65845 & -3.174144 \\
\hline $\mathrm{C}$ & -0.982919 & 0.212094 & -3.195862 \\
\hline $\mathrm{C}$ & -1.904004 & 0.872094 & -2.401119 \\
\hline $\mathrm{C}$ & -1.550981 & 2.068049 & -1.67797 \\
\hline $\mathrm{C}$ & 0.401862 & 3.425333 & -0.679031 \\
\hline $\mathrm{C}$ & 2.00443 & 2.898169 & -0.67017 \\
\hline $\mathrm{C}$ & 1.992328 & 1.756669 & -1.659814 \\
\hline $\mathrm{C}$ & 2.791363 & 0.654227 & -1.674683 \\
\hline $\mathrm{C}$ & 2.397636 & -0.513698 & -2.427998 \\
\hline $\mathrm{C}$ & 1.25313 & -0.512071 & -3.213163 \\
\hline $\mathrm{C}$ & -0.98926 & -1.239351 & -3.261831 \\
\hline $\mathrm{C}$ & -1.93975 & -1.918012 & -2.522285 \\
\hline $\mathrm{C}$ & -2.953283 & -1.205632 & -1.758998 \\
\hline $\mathrm{C}$ & -2.942962 & 0.154035 & -1.709091 \\
\hline $\mathrm{C}$ & -3.618825 & 1.056536 & -0.699617 \\
\hline $\mathrm{C}$ & -2.640441 & 2.426246 & -0.695695 \\
\hline $\mathrm{C}$ & -2.004537 & 2.898208 & 0.670175 \\
\hline $\mathrm{C}$ & -0.401955 & 3.425357 & 0.679022 \\
\hline $\mathrm{C}$ & 2.640332 & 2.426259 & 0.69564 \\
\hline $\mathrm{C}$ & 1.550899 & 2.068053 & 1.677875 \\
\hline $\mathrm{C}$ & 0.25666 & 2.492088 & 1.667227 \\
\hline $\mathrm{C}$ & -0.741804 & 1.729816 & 2.371722 \\
\hline $\mathrm{C}$ & -1.992445 & 1.756725 & 1.659894 \\
\hline $\mathrm{C}$ & -2.791439 & 0.654237 & 1.674753 \\
\hline $\mathrm{C}$ & -3.861602 & 0.298709 & 0.668057 \\
\hline $\mathrm{C}$ & -3.844701 & -1.371455 & 0.670711 \\
\hline $\mathrm{C}$ & -3.529467 & -2.142199 & -0.694711 \\
\hline
\end{tabular}




\begin{tabular}{|l|l|l|l|}
\hline C & 0.404161 & -1.692532 & -3.281168 \\
\hline C & -1.565845 & -3.124906 & -1.798817 \\
\hline C & -0.256745 & 2.492094 & -1.667291 \\
\hline F & 0.454324 & 4.725205 & -1.139462 \\
\hline F & -0.454319 & 4.725216 & 1.139505 \\
\hline F & -2.737831 & 3.975303 & 1.125627 \\
\hline F & -3.447776 & 3.44625 & -1.158084 \\
\hline F & 2.737786 & 3.975175 & -1.125659 \\
\hline F & 3.447544 & 3.446347 & 1.157993 \\
\hline F & -4.848411 & 1.496542 & -1.149617 \\
\hline F & -5.12328 & 0.649933 & 1.11105 \\
\hline F & -5.118528 & -1.749042 & 1.049592 \\
\hline F & -4.701882 & -2.746906 & -1.104286 \\
\hline F & 4.848336 & 1.496703 & 1.149494 \\
\hline F & 5.123222 & 0.650102 & -1.111113 \\
\hline F & 5.118612 & -1.748896 & -1.049554 \\
\hline F & 4.701964 & -2.746799 & 1.1043 \\
\hline
\end{tabular}

\begin{tabular}{|l|l|l|l|}
\hline $\mathrm{S}^{\prime}-\mathrm{C}_{60} \mathrm{~F}_{16}$ & & & \\
\hline $\mathrm{C}$ & 0.697754 & -3.337264 & -1.768878 \\
\hline $\mathrm{C}$ & 1.442895 & -3.632537 & -0.57122 \\
\hline $\mathrm{C}$ & 0.730011 & -3.831977 & 0.582244 \\
\hline $\mathrm{C}$ & -0.729969 & -3.831984 & 0.582224 \\
\hline $\mathrm{C}$ & -1.442826 & -3.63256 & -0.571262 \\
\hline $\mathrm{C}$ & -0.697682 & -3.337278 & -1.768911 \\
\hline $\mathrm{C}$ & 1.426668 & -2.308548 & -2.497929 \\
\hline
\end{tabular}




\begin{tabular}{|c|c|c|c|}
\hline $\mathrm{C}$ & 2.609643 & -1.939595 & -1.735571 \\
\hline $\mathrm{C}$ & 2.864499 & -3.00463 & -0.665497 \\
\hline $\mathrm{C}$ & 3.390038 & -2.359723 & 0.699323 \\
\hline $\mathrm{C}$ & 1.19177 & -3.158451 & 1.807616 \\
\hline $\bar{C}$ & -1.191799 & -3.158511 & 1.807604 \\
\hline $\mathrm{C}$ & -2.303451 & -2.354444 & 1.766606 \\
\hline $\mathrm{C}$ & -3.390011 & -2.359829 & 0.699254 \\
\hline $\mathrm{C}$ & -2.864416 & -3.004652 & -0.665579 \\
\hline $\mathrm{C}$ & -2.609453 & -1.939556 & -1.73555 \\
\hline $\mathrm{C}$ & -1.426558 & -2.308553 & -2.497957 \\
\hline $\mathrm{C}$ & -0.73237 & -1.372784 & -3.239141 \\
\hline $\mathrm{C}$ & 0.732504 & -1.372807 & -3.239169 \\
\hline $\mathrm{C}$ & 3.017408 & -0.641864 & -1.691919 \\
\hline $\mathrm{C}$ & 2.252737 & 0.358649 & -2.391327 \\
\hline $\mathrm{C}$ & 1.173964 & 0.010363 & -3.186066 \\
\hline $\mathrm{C}$ & 0.000072 & 0.862014 & -3.169395 \\
\hline $\mathrm{C}$ & -1.173834 & 0.010404 & -3.186041 \\
\hline $\mathrm{C}$ & -2.252621 & 0.358722 & -2.391304 \\
\hline $\mathrm{C}$ & -3.017192 & -0.641822 & -1.691855 \\
\hline $\mathrm{C}$ & -3.934246 & 0.013693 & -0.682293 \\
\hline $\mathrm{C}$ & -3.922896 & -0.774034 & 0.689699 \\
\hline $\mathrm{C}$ & -0.000026 & -2.763692 & 2.551846 \\
\hline $\mathrm{C}$ & -1.389982 & 1.564541 & 2.365437 \\
\hline $\mathrm{C}$ & -0.725432 & 0.655558 & 3.167499 \\
\hline $\mathrm{C}$ & 0.725155 & 0.655584 & 3.167547 \\
\hline $\mathrm{C}$ & 1.389681 & 1.564601 & 2.365529 \\
\hline $\mathrm{C}$ & 0.681031 & 2.59698 & 1.652408 \\
\hline
\end{tabular}




\begin{tabular}{|c|c|c|c|}
\hline $\mathrm{C}$ & -1.602813 & 3.274089 & 0.665907 \\
\hline $\mathrm{C}$ & -2.962634 & 2.273472 & 0.669435 \\
\hline $\mathrm{C}$ & -2.590974 & 1.197141 & 1.662693 \\
\hline $\mathrm{C}$ & -3.008386 & -0.099418 & 1.686169 \\
\hline $\mathrm{C}$ & -2.267111 & -1.084418 & 2.438887 \\
\hline $\mathrm{C}$ & -1.177017 & -0.722673 & 3.216104 \\
\hline $\mathrm{C}$ & 1.176833 & -0.722641 & 3.216154 \\
\hline $\mathrm{C}$ & 2.266983 & -1.084343 & 2.43895 \\
\hline $\mathrm{C}$ & 3.008231 & -0.0993 & 1.68625 \\
\hline $\mathrm{C}$ & 2.590736 & 1.19723 & 1.662756 \\
\hline $\mathrm{C}$ & 2.962475 & 2.273684 & 0.669775 \\
\hline $\mathrm{C}$ & 1.602591 & 3.274174 & 0.665978 \\
\hline $\mathrm{C}$ & 0.84496 & 3.515578 & -0.697223 \\
\hline $\mathrm{C}$ & -0.844862 & 3.515635 & -0.697258 \\
\hline $\mathrm{C}$ & -3.426684 & 1.62002 & -0.690442 \\
\hline $\mathrm{C}$ & -2.283239 & 1.610083 & -1.6767 \\
\hline $\mathrm{C}$ & -1.184145 & 2.415509 & -1.674823 \\
\hline $\mathrm{C}$ & 0.000094 & 1.995038 & -2.377614 \\
\hline $\mathrm{C}$ & 1.18432 & 2.41545 & -1.674741 \\
\hline $\mathrm{C}$ & 2.283367 & 1.609988 & -1.676578 \\
\hline $\mathrm{C}$ & 3.427035 & 1.619531 & -0.690549 \\
\hline $\mathrm{C}$ & 3.934242 & 0.013923 & -0.682205 \\
\hline $\mathrm{C}$ & 3.922783 & -0.773839 & 0.689814 \\
\hline $\mathrm{C}$ & -0.000076 & -1.580208 & 3.268158 \\
\hline $\mathrm{C}$ & 2.303386 & -2.354317 & 1.7666 \\
\hline $\mathrm{C}$ & -0.681339 & 2.59692 & 1.65233 \\
\hline $\mathrm{F}$ & -2.055226 & 4.49524 & 1.121607 \\
\hline
\end{tabular}




\begin{tabular}{|l|l|l|l|}
\hline F & -1.201975 & 4.763286 & -1.165437 \\
\hline F & 1.202217 & 4.763172 & -1.165376 \\
\hline F & 2.054823 & 4.495421 & 1.121574 \\
\hline F & -3.991754 & 3.071439 & 1.12637 \\
\hline F & -4.511706 & 2.336972 & -1.153162 \\
\hline F & 3.991628 & 3.071666 & 1.126575 \\
\hline F & 4.511834 & 2.33696 & -1.15316 \\
\hline F & 5.241832 & 0.049837 & -1.125573 \\
\hline F & 5.227932 & -0.828257 & 1.141739 \\
\hline F & -5.241649 & 0.050221 & -1.125975 \\
\hline F & -5.228012 & -0.828529 & 1.141699 \\
\hline F & -4.481731 & -3.107964 & 1.094508 \\
\hline F & -3.797378 & -3.940971 & -1.065399 \\
\hline F & 4.481805 & -3.107723 & 1.094656 \\
\hline F & 3.797478 & -3.940985 & -1.065176 \\
\hline
\end{tabular}

\begin{tabular}{|l|l|l|l|}
\hline $\mathrm{S}^{\prime}-\mathrm{C}_{60} \mathrm{~F}_{18}$ & & & \\
\hline $\mathrm{C}$ & 1.592418 & -2.79875 & -1.737751 \\
\hline $\mathrm{C}$ & 2.624265 & -3.138985 & -0.675801 \\
\hline $\mathrm{C}$ & 1.906094 & -3.585042 & 0.680045 \\
\hline $\mathrm{C}$ & 0.360229 & -3.768628 & 0.568308 \\
\hline $\mathrm{C}$ & -0.360372 & -3.768576 & -0.568308 \\
\hline $\mathrm{C}$ & 0.284213 & -3.219186 & -1.76175 \\
\hline $\mathrm{C}$ & 1.948796 & -1.581945 & -2.412433 \\
\hline $\mathrm{C}$ & 2.958537 & -0.868774 & -1.665461 \\
\hline $\mathrm{C}$ & 3.618775 & -1.789436 & -0.664953 \\
\hline
\end{tabular}




\begin{tabular}{|c|c|c|c|}
\hline $\mathrm{C}$ & 3.858317 & -1.038345 & 0.705665 \\
\hline $\mathrm{C}$ & 1.975685 & -2.480697 & 1.732391 \\
\hline $\mathrm{C}$ & -0.284372 & -3.219275 & 1.761781 \\
\hline $\mathrm{C}$ & -1.592546 & -2.798804 & 1.737787 \\
\hline $\mathrm{C}$ & -2.624349 & -3.138976 & 0.675782 \\
\hline $\mathrm{C}$ & -1.906184 & -3.584963 & -0.680042 \\
\hline $\mathrm{C}$ & -1.975693 & -2.480532 & -1.732329 \\
\hline $\mathrm{C}$ & -0.726055 & -2.459986 & -2.473747 \\
\hline $\mathrm{C}$ & -0.359985 & -1.354179 & -3.218165 \\
\hline $\mathrm{C}$ & 1.023099 & -0.903725 & -3.190156 \\
\hline $\mathrm{C}$ & 2.957946 & 0.493719 & -1.648532 \\
\hline $\mathrm{C}$ & 1.929987 & 1.211231 & -2.356822 \\
\hline $\mathrm{C}$ & 1.018793 & 0.547211 & -3.156971 \\
\hline $\mathrm{C}$ & -0.360786 & 0.996398 & -3.160722 \\
\hline $\mathrm{C}$ & -1.213162 & -0.177145 & -3.18378 \\
\hline $\mathrm{C}$ & -2.349881 & -0.184011 & -2.395859 \\
\hline $\mathrm{C}$ & -2.769264 & -1.37537 & -1.700226 \\
\hline $\mathrm{C}$ & -3.858284 & -1.038312 & -0.705637 \\
\hline $\mathrm{C}$ & -3.618827 & -1.789462 & 0.664894 \\
\hline $\mathrm{C}$ & 0.725934 & -2.459992 & 2.473683 \\
\hline $\mathrm{C}$ & -1.930064 & 1.211217 & 2.356802 \\
\hline $\mathrm{C}$ & -1.018877 & 0.547169 & 3.156952 \\
\hline $\mathrm{C}$ & 0.360696 & 0.996321 & 3.160772 \\
\hline $\mathrm{C}$ & 0.71604 & 2.073005 & 2.369832 \\
\hline $\mathrm{C}$ & -0.2758 & 2.839282 & 1.659851 \\
\hline $\mathrm{C}$ & -2.653588 & 2.78027 & 0.664071 \\
\hline $\mathrm{C}$ & -3.639036 & 1.407925 & 0.656913 \\
\hline
\end{tabular}




\begin{tabular}{|c|c|c|c|}
\hline $\mathrm{C}$ & -2.957958 & 0.49371 & 1.648563 \\
\hline $\mathrm{C}$ & -2.958513 & -0.868775 & 1.665382 \\
\hline $\mathrm{C}$ & -1.94891 & -1.581965 & 2.412426 \\
\hline $\mathrm{C}$ & -1.023208 & -0.903744 & 3.190144 \\
\hline $\mathrm{C}$ & 1.213055 & -0.177243 & 3.183791 \\
\hline $\mathrm{C}$ & 2.34973 & -0.184131 & 2.395782 \\
\hline $\mathrm{C}$ & 2.77249 & 0.995363 & 1.683546 \\
\hline $\mathrm{C}$ & 1.976834 & 2.102326 & 1.674781 \\
\hline $\mathrm{C}$ & 2.001465 & 3.251021 & 0.694276 \\
\hline $\mathrm{C}$ & 0.394157 & 3.776898 & 0.683711 \\
\hline $\mathrm{C}$ & -0.394206 & 3.776775 & -0.683589 \\
\hline $\mathrm{C}$ & -2.001354 & 3.250775 & -0.693906 \\
\hline $\mathrm{C}$ & -3.870693 & 0.648213 & -0.70715 \\
\hline $\mathrm{C}$ & -2.772434 & 0.995418 & -1.68359 \\
\hline $\mathrm{C}$ & -1.976719 & 2.102331 & -1.674718 \\
\hline $\mathrm{C}$ & -0.716044 & 2.07305 & -2.369717 \\
\hline $\mathrm{C}$ & 0.275804 & 2.839268 & -1.659694 \\
\hline $\mathrm{C}$ & 1.571487 & 2.415799 & -1.65238 \\
\hline $\mathrm{C}$ & 2.654119 & 2.780203 & -0.664359 \\
\hline $\mathrm{C}$ & 3.639107 & 1.408206 & -0.657172 \\
\hline $\mathrm{C}$ & 3.870856 & 0.647795 & 0.707364 \\
\hline $\mathrm{C}$ & 0.359858 & -1.354228 & 3.218172 \\
\hline $\mathrm{C}$ & 2.769234 & -1.375502 & 1.700211 \\
\hline $\mathrm{C}$ & -1.571482 & 2.415738 & 1.652446 \\
\hline $\mathrm{F}$ & -3.4629 & 3.799379 & 1.121641 \\
\hline $\mathrm{F}$ & -2.725812 & 4.327006 & -1.162469 \\
\hline $\mathrm{F}$ & -0.44063 & 5.074947 & -1.147178 \\
\hline
\end{tabular}




\begin{tabular}{|l|l|l|l|}
\hline F & 0.440345 & 5.075165 & 1.147128 \\
\hline F & -4.866109 & 1.847886 & 1.109264 \\
\hline F & -5.120449 & 0.996836 & -1.177437 \\
\hline F & 2.725901 & 4.327297 & 1.16281 \\
\hline F & 3.463135 & 3.79961 & -1.121943 \\
\hline F & 4.866567 & 1.847548 & -1.109237 \\
\hline F & 5.120624 & 0.996663 & 1.177434 \\
\hline F & -5.107209 & -1.404088 & -1.167042 \\
\hline F & -4.846426 & -2.242293 & 1.10832 \\
\hline F & -3.436578 & -4.186058 & 1.061336 \\
\hline F & -2.505709 & -4.756027 & -1.100211 \\
\hline F & 5.107162 & -1.404699 & 1.166777 \\
\hline F & 4.846446 & -2.242265 & -1.108124 \\
\hline F & 3.436463 & -4.186069 & -1.061406 \\
\hline F & 2.505597 & -4.756139 & 1.100068 \\
\hline
\end{tabular}

\begin{tabular}{|l|l|l|l|}
\hline $\mathrm{S}^{\prime}-\mathrm{C}_{60} \mathrm{~F}_{20}$ & & & \\
\hline $\mathrm{C}$ & 0.977237 & 2.873748 & 1.65742 \\
\hline $\mathrm{C}$ & 1.964926 & 3.46809 & 0.681696 \\
\hline $\mathrm{C}$ & 1.235764 & 3.789673 & -0.681794 \\
\hline $\mathrm{C}$ & -0.448865 & 3.960745 & -0.681723 \\
\hline $\mathrm{C}$ & -1.227781 & 3.792244 & 0.681715 \\
\hline $\mathrm{C}$ & -0.379668 & 3.011497 & 1.657416 \\
\hline $\mathrm{C}$ & 1.580598 & 1.767821 & 2.35644 \\
\hline $\mathrm{C}$ & 2.746992 & 1.291659 & 1.657475 \\
\hline $\mathrm{C}$ & 3.227287 & 2.339521 & 0.681686 \\
\hline
\end{tabular}




\begin{tabular}{|c|c|c|c|}
\hline $\mathrm{C}$ & 3.628205 & 1.650727 & -0.681748 \\
\hline $\mathrm{C}$ & 1.462998 & 2.659533 & -1.657471 \\
\hline $\mathrm{C}$ & -0.898665 & 2.899382 & -1.657463 \\
\hline $\mathrm{C}$ & -2.077365 & 2.213271 & -1.657465 \\
\hline $\mathrm{C}$ & -3.222331 & 2.346346 & -0.681591 \\
\hline $\mathrm{C}$ & -2.691223 & 2.940428 & 0.681767 \\
\hline $\mathrm{C}$ & -2.43122 & 1.81741 & 1.657569 \\
\hline $\mathrm{C}$ & -1.192949 & 2.049466 & 2.356536 \\
\hline $\mathrm{C}$ & -0.620947 & 1.066823 & 3.142357 \\
\hline $\mathrm{C}$ & 0.822839 & 0.920218 & 3.142301 \\
\hline $\mathrm{C}$ & 3.035222 & -0.041387 & 1.657396 \\
\hline $\mathrm{C}$ & 2.169913 & -0.956928 & 2.356494 \\
\hline $\mathrm{C}$ & 1.129608 & -0.498169 & 3.142341 \\
\hline $\mathrm{C}$ & -0.124582 & -1.228155 & 3.142372 \\
\hline $\mathrm{C}$ & -1.206478 & -0.260997 & 3.142415 \\
\hline $\mathrm{C}$ & -2.317886 & -0.501337 & 2.356696 \\
\hline $\mathrm{C}$ & -2.981506 & 0.569482 & 1.657592 \\
\hline $\mathrm{C}$ & -3.98592 & 0.004116 & 0.681639 \\
\hline $\mathrm{C}$ & -3.905578 & 0.797134 & -0.681607 \\
\hline $\mathrm{C}$ & 0.239489 & 2.359181 & -2.356423 \\
\hline $\mathrm{C}$ & -1.580745 & -1.767941 & -2.356664 \\
\hline $\mathrm{C}$ & -0.822861 & -0.920264 & -3.142391 \\
\hline $\mathrm{C}$ & 0.620867 & -1.066893 & -3.14238 \\
\hline $\mathrm{C}$ & 1.192852 & -2.049571 & -2.356602 \\
\hline $\mathrm{C}$ & 0.379521 & -3.01155 & -1.657631 \\
\hline $\mathrm{C}$ & -1.964657 & -3.467732 & -0.681269 \\
\hline $\mathrm{C}$ & -3.227149 & -2.339216 & -0.681288 \\
\hline
\end{tabular}




\begin{tabular}{|c|c|c|c|}
\hline $\mathrm{C}$ & -2.746982 & -1.291707 & -1.65762 \\
\hline $\mathrm{C}$ & -3.035177 & 0.041374 & -1.65747 \\
\hline $\mathrm{C}$ & -2.169894 & 0.9569 & -2.356533 \\
\hline $\mathrm{C}$ & -1.129585 & 0.498093 & -3.142347 \\
\hline $\bar{C}$ & 1.206455 & 0.260841 & -3.142298 \\
\hline $\mathrm{C}$ & 2.317743 & 0.501127 & -2.356451 \\
\hline $\mathrm{C}$ & 2.981524 & -0.569703 & -1.657565 \\
\hline $\mathrm{C}$ & 2.431111 & -1.817552 & -1.657581 \\
\hline $\mathrm{C}$ & 2.691001 & -2.940459 & -0.681658 \\
\hline $\mathrm{C}$ & 1.227193 & -3.79213 & -0.681376 \\
\hline $\mathrm{C}$ & 0.448993 & -3.960242 & 0.681259 \\
\hline $\mathrm{C}$ & -1.235675 & -3.789156 & 0.681271 \\
\hline $\mathrm{C}$ & -3.627797 & -1.651106 & 0.681468 \\
\hline $\mathrm{C}$ & -2.479729 & -1.750648 & 1.657745 \\
\hline $\mathrm{C}$ & -1.462851 & -2.659587 & 1.657643 \\
\hline $\mathrm{C}$ & -0.239411 & -2.359356 & 2.356587 \\
\hline $\mathrm{C}$ & 0.898697 & -2.899362 & 1.657559 \\
\hline $\mathrm{C}$ & 2.07743 & -2.21327 & 1.657481 \\
\hline $\mathrm{C}$ & 3.222421 & -2.346323 & 0.681607 \\
\hline $\mathrm{C}$ & 3.905613 & -0.797098 & 0.681549 \\
\hline $\mathrm{C}$ & 3.986083 & -0.004302 & -0.681743 \\
\hline $\mathrm{C}$ & 0.124607 & 1.228049 & -3.142251 \\
\hline $\mathrm{C}$ & 2.479729 & 1.750469 & -1.657415 \\
\hline $\mathrm{C}$ & -0.977396 & -2.873769 & -1.657671 \\
\hline $\mathrm{F}$ & -2.531048 & -4.635339 & -1.147506 \\
\hline $\mathrm{F}$ & -1.715782 & -4.994775 & 1.147576 \\
\hline $\mathrm{F}$ & 0.677003 & -5.23769 & 1.147689 \\
\hline
\end{tabular}




\begin{tabular}{|l|l|l|l|}
\hline F & 1.547015 & -5.049792 & -1.147661 \\
\hline F & -4.324145 & -3.032458 & -1.147776 \\
\hline F & -4.772245 & -2.262901 & 1.147866 \\
\hline F & 3.626068 & -3.840168 & -1.148306 \\
\hline F & 4.220401 & -3.175731 & 1.148172 \\
\hline F & 5.191181 & -0.974565 & 1.147888 \\
\hline F & 5.281035 & -0.088756 & -1.148098 \\
\hline F & -5.280824 & 0.087957 & 1.148332 \\
\hline F & -5.191007 & 0.974774 & -1.148167 \\
\hline F & -4.220311 & 3.17594 & -1.147901 \\
\hline F & -3.626865 & 3.839693 & 1.148053 \\
\hline F & 4.772469 & 2.262799 & -1.148052 \\
\hline F & 4.324609 & 3.032279 & 1.147898 \\
\hline F & 2.530908 & 4.635879 & 1.148014 \\
\hline F & 1.716366 & 4.995087 & -1.148077 \\
\hline F & -0.677363 & 5.23817 & -1.147987 \\
\hline F & -1.547494 & 5.04996 & 1.148046 \\
\hline
\end{tabular}

\begin{tabular}{|l|l|l|l|}
\hline$T^{\prime}-\mathrm{C}_{60} \mathrm{~F}_{6}$ & & & \\
\hline $\mathrm{C}$ & 3.35499548 & 1.52986151 & 1.19255750 \\
\hline $\mathrm{C}$ & 3.11940925 & 0.80143873 & 2.44019114 \\
\hline $\mathrm{C}$ & 3.36522633 & -1.31940582 & 1.20038122 \\
\hline $\mathrm{C}$ & 3.12448460 & -0.58387079 & 2.44376754 \\
\hline $\mathrm{C}$ & 1.15614427 & 0.84123635 & 3.93365825 \\
\hline $\mathrm{C}$ & 2.11362874 & -1.31521854 & 3.20952320 \\
\hline $\mathrm{C}$ & 2.11053089 & 1.53390481 & 3.20683866 \\
\hline
\end{tabular}




\begin{tabular}{|c|c|c|c|}
\hline $\mathrm{C}$ & 1.15674832 & -0.62249455 & 3.93343348 \\
\hline $\mathrm{C}$ & 1.73125288 & -2.49760059 & 2.43576615 \\
\hline $\mathrm{C}$ & 1.72666759 & 2.71952096 & 2.43934081 \\
\hline $\mathrm{C}$ & -1.09607461 & 0.11046151 & 3.92915656 \\
\hline $\mathrm{C}$ & -0.23550468 & 1.29491441 & 3.93258425 \\
\hline $\mathrm{C}$ & 0.41507690 & -2.92288125 & 2.43013650 \\
\hline $\mathrm{C}$ & 2.50170803 & -2.49383927 & 1.19023093 \\
\hline $\mathrm{C}$ & -0.19881074 & -3.35165088 & 1.17259592 \\
\hline $\mathrm{C}$ & -0.59687193 & -2.19449514 & 3.19841915 \\
\hline $\mathrm{C}$ & -1.83572542 & -2.18685049 & 2.41918603 \\
\hline $\mathrm{C}$ & -0.23591888 & -1.07414414 & 3.92967957 \\
\hline $\mathrm{C}$ & -1.84003512 & 2.42077798 & 2.43358354 \\
\hline $\mathrm{C}$ & 0.41097112 & 3.15317776 & 2.44211117 \\
\hline $\mathrm{C}$ & -1.57970369 & -2.89507368 & 1.16401506 \\
\hline $\mathrm{C}$ & -2.64871447 & -1.06720064 & 2.41869149 \\
\hline $\mathrm{C}$ & -3.24235980 & -0.60524900 & 1.16300214 \\
\hline $\mathrm{C}$ & -2.27281815 & 0.11362345 & 3.19739348 \\
\hline $\mathrm{C}$ & -2.65185044 & 1.30089631 & 2.42826330 \\
\hline $\mathrm{C}$ & -0.21338887 & 3.60906132 & 1.19819494 \\
\hline $\mathrm{C}$ & 2.49276579 & 2.71732472 & 1.19203007 \\
\hline $\mathrm{C}$ & -0.59769201 & 2.41881052 & 3.20786880 \\
\hline $\mathrm{C}$ & -1.59743769 & 3.15150520 & 1.18772566 \\
\hline $\mathrm{C}$ & -3.24905524 & 0.84915651 & 1.17073407 \\
\hline $\mathrm{C}$ & 2.17458812 & -2.21300725 & -1.24803904 \\
\hline $\mathrm{C}$ & -2.12270283 & -2.43839442 & -0.02524084 \\
\hline $\mathrm{C}$ & 1.89707114 & 3.14292041 & 0.01822460 \\
\hline $\mathrm{C}$ & 2.52100160 & 0.09816856 & -1.96796467 \\
\hline
\end{tabular}




\begin{tabular}{|c|c|c|c|}
\hline $\mathrm{C}$ & -0.08466741 & -2.88338621 & -1.25802758 \\
\hline $\mathrm{C}$ & 3.58120135 & -0.63156361 & 0.01689894 \\
\hline $\mathrm{C}$ & 0.52111769 & -3.32351662 & -0.00975008 \\
\hline $\mathrm{C}$ & 1.00742132 & -2.45913761 & -2.24064964 \\
\hline $\mathrm{C}$ & 1.91431334 & -2.90295331 & -0.00025712 \\
\hline $\mathrm{C}$ & 3.57578159 & 0.83099882 & 0.01925208 \\
\hline $\mathrm{C}$ & 0.50802450 & 3.60081504 & 0.01501748 \\
\hline $\mathrm{C}$ & -0.12476752 & 3.15013614 & -1.22354928 \\
\hline $\mathrm{C}$ & -2.16777878 & 2.71890813 & -0.00275411 \\
\hline $\mathrm{C}$ & -1.43062540 & 2.75209650 & -1.25024573 \\
\hline $\mathrm{C}$ & 0.48854357 & 1.23104016 & -2.64848812 \\
\hline $\mathrm{C}$ & -2.02555309 & 1.71787573 & -2.24250847 \\
\hline $\mathrm{C}$ & 0.87456398 & 2.36564674 & -1.96897765 \\
\hline $\mathrm{C}$ & -0.92171153 & 0.93303344 & -3.10769113 \\
\hline $\mathrm{C}$ & -2.76742919 & 0.81131996 & -1.25966620 \\
\hline $\mathrm{C}$ & 2.11107527 & 2.38947355 & -1.21808497 \\
\hline $\mathrm{C}$ & 0.60319408 & -1.16706898 & -3.10675433 \\
\hline $\mathrm{C}$ & 1.32291485 & 0.08193872 & -2.64796627 \\
\hline $\mathrm{C}$ & -2.75116270 & -0.54312673 & -1.26822194 \\
\hline $\mathrm{C}$ & -2.99894447 & 1.52432198 & -0.01189976 \\
\hline $\mathrm{C}$ & -2.97569751 & -1.26363940 & -0.02576009 \\
\hline $\mathrm{C}$ & -2.00368763 & -1.45590488 & -2.25527563 \\
\hline $\mathrm{C}$ & -1.36754965 & -2.44865281 & -1.26737922 \\
\hline $\mathrm{C}$ & -0.96360513 & -0.70111653 & -3.18365216 \\
\hline $\mathrm{C}$ & 2.95729868 & -1.09448588 & -1.22166150 \\
\hline $\mathrm{C}$ & 2.92611296 & 1.26700697 & -1.21758571 \\
\hline $\mathrm{F}$ & -2.89362208 & -2.10246053 & -3.09953107 \\
\hline
\end{tabular}




\begin{tabular}{|l|l|l|l|}
\hline $\mathrm{F}$ & -1.36009777 & -0.98960260 & -4.48072477 \\
\hline $\mathrm{F}$ & 1.31779640 & -3.46210252 & -3.13917534 \\
\hline $\mathrm{F}$ & 0.95304689 & -1.48126832 & -4.41291276 \\
\hline $\mathrm{F}$ & -2.88280572 & 2.32295956 & -3.14174325 \\
\hline $\mathrm{F}$ & -1.11164416 & 1.36217851 & -4.41418109 \\
\hline
\end{tabular}

\begin{tabular}{|l|l|l|l|}
\hline${ }^{\prime}{ }^{\prime}-\mathrm{C}_{60} \mathrm{~F}_{8}$ & & & \\
\hline $\mathrm{C}$ & 3.40783491 & 1.33779128 & 1.29001474 \\
\hline $\mathrm{C}$ & 3.17690616 & 0.60678889 & 2.53725063 \\
\hline $\mathrm{C}$ & 3.41537965 & -1.51387460 & 1.29025804 \\
\hline $\mathrm{C}$ & 3.17768893 & -0.77909625 & 2.53527222 \\
\hline $\mathrm{C}$ & 1.21309664 & 0.64833635 & 4.02724662 \\
\hline $\mathrm{C}$ & 2.16507554 & -1.50951039 & 3.29904670 \\
\hline $\mathrm{C}$ & 2.17154357 & 1.34111538 & 3.30696855 \\
\hline $\mathrm{C}$ & 1.20947550 & -0.81509303 & 4.02369626 \\
\hline $\mathrm{C}$ & 1.78035137 & -2.69023555 & 2.52393523 \\
\hline $\mathrm{C}$ & 1.79169787 & 2.53014072 & 2.53998616 \\
\hline $\mathrm{C}$ & -1.04109176 & -0.07795978 & 4.01789973 \\
\hline $\mathrm{C}$ & -0.17804399 & 1.10603058 & 4.01990427 \\
\hline $\mathrm{C}$ & 0.46379943 & -3.11414321 & 2.51801767 \\
\hline $\mathrm{C}$ & 2.55036274 & -2.68708102 & 1.27803859 \\
\hline $\mathrm{C}$ & -0.15063391 & -3.54400550 & 1.26070535 \\
\hline $\mathrm{C}$ & -0.54669724 & -2.38433243 & 3.28623396 \\
\hline $\mathrm{C}$ & -1.78549722 & -2.37608868 & 2.50690139 \\
\hline $\mathrm{C}$ & -0.18385947 & -1.26421969 & 4.01814086 \\
\hline & -1.76751011 & 2.23090647 & 2.50629394 \\
\hline & & & \\
\hline & & & \\
\hline & & & \\
\hline & & & \\
\hline & & & \\
\hline & & & \\
\hline & & & \\
\hline & & & \\
\hline & & & \\
\hline & & & \\
\hline
\end{tabular}




\begin{tabular}{|c|c|c|c|}
\hline $\mathrm{C}$ & 0.47483958 & 2.96496844 & 2.52881153 \\
\hline $\mathrm{C}$ & -1.53114458 & -3.08597816 & 1.25164065 \\
\hline $\mathrm{C}$ & -2.59536858 & -1.25411401 & 2.50700047 \\
\hline $\mathrm{C}$ & -3.17669682 & -0.78829873 & 1.24670923 \\
\hline $\mathrm{C}$ & -2.21735733 & -0.07398543 & 3.28664742 \\
\hline $\mathrm{C}$ & -2.58380637 & 1.11259099 & 2.50926627 \\
\hline $\mathrm{C}$ & -0.13623985 & 3.43802665 & 1.28752311 \\
\hline $\mathrm{C}$ & 2.54700348 & 2.52621449 & 1.29448614 \\
\hline $\mathrm{C}$ & -0.53322141 & 2.22836031 & 3.28993478 \\
\hline $\mathrm{C}$ & -1.53204130 & 2.96201749 & 1.26469143 \\
\hline $\mathrm{C}$ & -3.15872548 & 0.66456097 & 1.25009633 \\
\hline $\mathrm{C}$ & 2.22039773 & -2.40667592 & -1.15986534 \\
\hline $\mathrm{C}$ & -2.07271558 & -2.63119786 & 0.06053146 \\
\hline $\mathrm{C}$ & 1.93109975 & 2.95540906 & 0.12738908 \\
\hline $\mathrm{C}$ & 2.54101239 & -0.09133404 & -1.85777869 \\
\hline $\mathrm{C}$ & -0.03731119 & -3.08033892 & -1.17139609 \\
\hline $\mathrm{C}$ & 3.62893211 & -0.82390578 & 0.10724479 \\
\hline $\mathrm{C}$ & 0.56868497 & -3.51806095 & 0.07796932 \\
\hline $\mathrm{C}$ & 1.05116033 & -2.64845936 & -2.15319453 \\
\hline $\mathrm{C}$ & 1.96215645 & -3.09711341 & 0.08752394 \\
\hline $\mathrm{C}$ & 3.61855700 & 0.63879559 & 0.11607186 \\
\hline $\mathrm{C}$ & 0.57250682 & 3.46146627 & 0.11930700 \\
\hline $\mathrm{C}$ & -0.03598905 & 3.28753790 & -1.30195950 \\
\hline $\mathrm{C}$ & -2.08314896 & 2.54173348 & 0.08143976 \\
\hline $\mathrm{C}$ & -1.57238976 & 2.86368502 & -1.31751572 \\
\hline $\mathrm{C}$ & 0.49044006 & 1.04642710 & -2.49469548 \\
\hline $\mathrm{C}$ & -2.01044583 & 1.52303440 & -2.20339400 \\
\hline
\end{tabular}




\begin{tabular}{|c|c|c|c|}
\hline $\mathrm{C}$ & 0.87408838 & 2.17719850 & -1.83142221 \\
\hline $\mathrm{C}$ & -0.88641062 & 0.75369488 & -3.02246244 \\
\hline $\mathrm{C}$ & -2.65536655 & 0.63032892 & -1.16956944 \\
\hline $\mathrm{C}$ & 2.11276293 & 2.18282602 & -1.09384323 \\
\hline $\mathrm{C}$ & 0.63836337 & -1.34745634 & -3.00849451 \\
\hline $\mathrm{C}$ & 1.33399757 & -0.10220996 & -2.51430203 \\
\hline $\mathrm{C}$ & -2.67606204 & -0.72559868 & -1.17928815 \\
\hline $\mathrm{C}$ & -2.86919296 & 1.33957997 & 0.07085606 \\
\hline $\mathrm{C}$ & -2.91238781 & -1.44723175 & 0.05956689 \\
\hline $\mathrm{C}$ & -1.95497859 & -1.64929537 & -2.17140798 \\
\hline $\mathrm{C}$ & -1.32016250 & -2.64617436 & -1.18358550 \\
\hline $\mathrm{C}$ & -0.92503213 & -0.89041876 & -3.10203772 \\
\hline $\mathrm{C}$ & 3.00018303 & -1.28570027 & -1.12954678 \\
\hline $\mathrm{C}$ & 2.94292809 & 1.07442262 & -1.10512032 \\
\hline $\mathrm{F}$ & -2.85655585 & -2.29020632 & -3.00662110 \\
\hline $\mathrm{F}$ & -1.31642202 & -1.17548988 & -4.39969395 \\
\hline $\mathrm{F}$ & 1.36251236 & -3.64125515 & -3.06152721 \\
\hline $\mathrm{F}$ & 1.01453960 & -1.63873397 & -4.31262077 \\
\hline $\mathrm{F}$ & -2.93232391 & 1.98112487 & -3.12745473 \\
\hline $\mathrm{F}$ & -1.01526206 & 1.17798270 & -4.33785884 \\
\hline $\mathrm{F}$ & 0.07806991 & 4.45151893 & -2.03834205 \\
\hline $\mathrm{F}$ & -2.26975626 & 3.91852513 & -1.87564051 \\
\hline
\end{tabular}

\begin{tabular}{|l|l|l|l|}
\hline 'bis' $-\mathrm{C}_{60} \mathrm{~F}_{8}$ & & & \\
\hline $\mathrm{C}$ & 3.31837 & -1.18643 & -2.10285 \\
\hline $\mathrm{C}$ & 3.934 & -0.00013 & -1.50175 \\
\hline
\end{tabular}




\begin{tabular}{|c|c|c|c|}
\hline $\mathrm{C}$ & 4.26655 & -0.00012 & -0.15868 \\
\hline $\mathrm{C}$ & 4.00364 & -1.18481 & 0.6606 \\
\hline $\mathrm{C}$ & 3.41774 & -2.30318 & 0.09206 \\
\hline $\mathrm{C}$ & 3.06639 & -2.31028 & -1.32869 \\
\hline $\bar{C}$ & 2.31768 & -0.73193 & -3.06581 \\
\hline $\mathrm{C}$ & 2.31785 & 0.73191 & -3.06593 \\
\hline $\mathrm{C}$ & 3.31859 & 1.18628 & -2.10299 \\
\hline $\mathrm{C}$ & 3.06657 & 2.31012 & -1.32875 \\
\hline $\mathrm{C}$ & 4.00369 & 1.18455 & 0.66063 \\
\hline $\mathrm{C}$ & 3.5883 & -0.73162 & 1.99035 \\
\hline $\mathrm{C}$ & 2.60926 & -1.42516 & 2.68322 \\
\hline $\mathrm{C}$ & 1.98485 & -2.60266 & 2.07505 \\
\hline $\mathrm{C}$ & 2.37314 & -3.0282 & 0.81541 \\
\hline $\mathrm{C}$ & 1.36872 & -3.48462 & -0.13906 \\
\hline $\mathrm{C}$ & 1.81314 & -3.04602 & -1.47977 \\
\hline $\mathrm{C}$ & 0.8599 & -2.61613 & -2.36929 \\
\hline $\mathrm{C}$ & 1.12174 & -1.42796 & -3.1791 \\
\hline $\mathrm{C}$ & 1.12189 & 1.42801 & -3.17902 \\
\hline $\mathrm{C}$ & -0.12724 & 0.68252 & -3.24423 \\
\hline $\mathrm{C}$ & -0.12731 & -0.68248 & -3.24433 \\
\hline $\mathrm{C}$ & -1.12599 & -1.42671 & -2.44253 \\
\hline $\mathrm{C}$ & -0.57182 & -2.59239 & -1.98095 \\
\hline $\mathrm{C}$ & -1.11586 & -3.38581 & -0.82907 \\
\hline $\mathrm{C}$ & 0.03238 & -3.49351 & 0.18829 \\
\hline $\mathrm{C}$ & -0.37825 & -2.96929 & 1.45941 \\
\hline $\mathrm{C}$ & 0.56707 & -2.58174 & 2.40287 \\
\hline $\mathrm{C}$ & 3.58833 & 0.73138 & 1.9904 \\
\hline
\end{tabular}




\begin{tabular}{|c|c|c|c|}
\hline $\mathrm{C}$ & -0.87892 & 0.72548 & 3.03741 \\
\hline $\mathrm{C}$ & 0.3075 & 1.41192 & 3.22504 \\
\hline $\mathrm{C}$ & 0.56717 & 2.58172 & 2.40299 \\
\hline $\mathrm{C}$ & -0.37809 & 2.96936 & 1.45947 \\
\hline $\bar{C}$ & -1.63497 & 2.26349 & 1.32051 \\
\hline $\mathrm{C}$ & -2.82175 & 0.00003 & 1.75993 \\
\hline $\mathrm{C}$ & -1.8847 & -1.17461 & 2.08915 \\
\hline $\mathrm{C}$ & -0.87889 & -0.72537 & 3.03727 \\
\hline $\mathrm{C}$ & 0.30749 & -1.41189 & 3.22495 \\
\hline $\mathrm{C}$ & 1.56949 & -0.69197 & 3.40671 \\
\hline $\mathrm{C}$ & 1.56949 & 0.69189 & 3.40673 \\
\hline $\mathrm{C}$ & 1.98491 & 2.6024 & 2.07504 \\
\hline $\mathrm{C}$ & 2.37327 & 3.02803 & 0.81546 \\
\hline $\mathrm{C}$ & 1.369 & 3.48472 & -0.13904 \\
\hline $\mathrm{C}$ & 0.03261 & 3.49386 & 0.18835 \\
\hline $\mathrm{C}$ & -1.11572 & 3.38588 & -0.82906 \\
\hline $\mathrm{C}$ & -2.31301 & 2.59596 & 0.0123 \\
\hline $\mathrm{C}$ & -2.97945 & 1.30314 & -0.65763 \\
\hline $\mathrm{C}$ & -3.35728 & 0.00009 & 0.2765 \\
\hline $\mathrm{C}$ & -1.63511 & -2.26353 & 1.32052 \\
\hline $\mathrm{C}$ & -2.31316 & -2.59595 & 0.01224 \\
\hline $\mathrm{C}$ & -2.97945 & -1.30301 & -0.65761 \\
\hline $\mathrm{C}$ & -2.11626 & -0.68118 & -1.72709 \\
\hline $\mathrm{C}$ & -2.11639 & 0.68129 & -1.72725 \\
\hline $\mathrm{C}$ & -1.12589 & 1.42672 & -2.4425 \\
\hline $\mathrm{C}$ & -0.57157 & 2.59226 & -1.9808 \\
\hline $\mathrm{C}$ & 0.8601 & 2.61638 & -2.36945 \\
\hline
\end{tabular}




\begin{tabular}{|l|l|l|l|}
\hline C & 1.81339 & 3.04612 & -1.47987 \\
\hline C & 2.60925 & 1.42497 & 2.68324 \\
\hline C & 3.41776 & 2.3029 & 0.09207 \\
\hline C & -1.88469 & 1.17464 & 2.08916 \\
\hline F & -3.92984 & 0. & 2.5928 \\
\hline F & -4.73695 & 0.0002 & 0.36487 \\
\hline F & -4.18828 & 1.73703 & -1.1805 \\
\hline F & -3.32993 & 3.51727 & 0.17905 \\
\hline F & -4.1882 & -1.73685 & -1.1808 \\
\hline F & -3.3301 & -3.51728 & 0.17897 \\
\hline F & -1.61828 & -4.60895 & -1.21062 \\
\hline F & -1.61805 & 4.60903 & -1.21059 \\
\hline
\end{tabular}

\begin{tabular}{|l|l|l|l|}
\hline$' T '-\mathrm{C}_{60} \mathrm{~F}_{10}$ & & & \\
\hline $\mathrm{C}$ & 3.37359945 & 1.12669637 & 1.26611028 \\
\hline $\mathrm{C}$ & 3.15362185 & 0.39256207 & 2.51107033 \\
\hline $\mathrm{C}$ & 3.40184349 & -1.72804705 & 1.26131726 \\
\hline $\mathrm{C}$ & 3.16168281 & -0.99307384 & 2.50693062 \\
\hline $\mathrm{C}$ & 1.20200366 & 0.42606964 & 4.00928806 \\
\hline $\mathrm{C}$ & 2.15216288 & -1.72931748 & 3.27098312 \\
\hline $\mathrm{C}$ & 2.15251155 & 1.12714608 & 3.28343116 \\
\hline $\mathrm{C}$ & 1.19894666 & -1.03879561 & 4.00119760 \\
\hline $\mathrm{C}$ & 1.76733828 & -2.90694088 & 2.49176203 \\
\hline $\mathrm{C}$ & 1.77191160 & 2.32576843 & 2.53730192 \\
\hline $\mathrm{C}$ & -1.04804345 & -0.29669701 & 3.98886840 \\
\hline $\mathrm{C}$ & -0.18106072 & 0.88444912 & 3.99361368 \\
\hline
\end{tabular}




\begin{tabular}{|c|c|c|c|}
\hline $\mathrm{C}$ & 0.45037791 & -3.33077887 & 2.48374467 \\
\hline $\mathrm{C}$ & 2.53743427 & -2.90139006 & 1.24608976 \\
\hline $\mathrm{C}$ & -0.16334069 & -3.75770630 & 1.22509711 \\
\hline $\mathrm{C}$ & -0.55975906 & -2.60257634 & 3.25342504 \\
\hline $\mathrm{C}$ & -1.79738939 & -2.59087811 & 2.47159759 \\
\hline $\mathrm{C}$ & -0.19510164 & -1.48611668 & 3.99096852 \\
\hline $\mathrm{C}$ & -1.72890491 & 2.00238903 & 2.44703298 \\
\hline $\mathrm{C}$ & 0.48106714 & 2.77832602 & 2.53727051 \\
\hline $\mathrm{C}$ & -1.54357857 & -3.29867413 & 1.21504934 \\
\hline $\mathrm{C}$ & -2.60416066 & -1.46639609 & 2.47122790 \\
\hline $\mathrm{C}$ & -3.18037131 & -0.99863632 & 1.20893998 \\
\hline $\mathrm{C}$ & -2.21881592 & -0.28853750 & 3.25086319 \\
\hline $\mathrm{C}$ & -2.56061833 & 0.89784830 & 2.46378039 \\
\hline $\mathrm{C}$ & -0.19958406 & 3.56711240 & 1.38276298 \\
\hline $\mathrm{C}$ & 2.52168694 & 2.31734919 & 1.26808398 \\
\hline $\mathrm{C}$ & -0.51769853 & 2.01084610 & 3.25322354 \\
\hline $\mathrm{C}$ & -1.48012289 & 2.74353534 & 1.22074791 \\
\hline $\mathrm{C}$ & -3.13919598 & 0.45304785 & 1.20826342 \\
\hline $\mathrm{C}$ & 2.20891558 & -2.62011819 & -1.19301729 \\
\hline $\mathrm{C}$ & -2.08426441 & -2.84368141 & 0.02309618 \\
\hline $\mathrm{C}$ & 1.90078971 & 2.74667444 & 0.12523326 \\
\hline $\mathrm{C}$ & 2.51384892 & -0.30201249 & -1.87592173 \\
\hline $\mathrm{C}$ & -0.04909414 & -3.29343920 & -1.20733106 \\
\hline $\mathrm{C}$ & 3.61577783 & -1.03775275 & 0.07917430 \\
\hline $\mathrm{C}$ & 0.55654225 & -3.73097625 & 0.04277811 \\
\hline $\mathrm{C}$ & 1.03937601 & -2.86066083 & -2.18812801 \\
\hline $\mathrm{C}$ & 1.94997255 & -3.31084904 & 0.05416203 \\
\hline
\end{tabular}




\begin{tabular}{|c|c|c|c|}
\hline $\mathrm{C}$ & 3.59104871 & 0.42526942 & 0.09360779 \\
\hline $\mathrm{C}$ & 0.65348832 & 3.61797078 & 0.03328759 \\
\hline $\mathrm{C}$ & -0.06237345 & 3.10116906 & -1.38504606 \\
\hline $\mathrm{C}$ & -2.02074407 & 2.31749239 & 0.04806203 \\
\hline $\mathrm{C}$ & -1.57867319 & 2.66669733 & -1.35660096 \\
\hline $\mathrm{C}$ & 0.45992433 & 0.82939602 & -2.50068139 \\
\hline $\mathrm{C}$ & -2.02306385 & 1.31715070 & -2.24797766 \\
\hline $\mathrm{C}$ & 0.82189050 & 1.95181206 & -1.80827701 \\
\hline $\mathrm{C}$ & -0.90282353 & 0.54254374 & -3.06119889 \\
\hline $\mathrm{C}$ & -2.65005363 & 0.42009114 & -1.20924075 \\
\hline $\mathrm{C}$ & 2.04572568 & 1.95457924 & -1.06583347 \\
\hline $\mathrm{C}$ & 0.62296298 & -1.55807544 & -3.04184737 \\
\hline $\mathrm{C}$ & 1.30777936 & -0.31458502 & -2.53160234 \\
\hline $\mathrm{C}$ & -2.68447809 & -0.93658996 & -1.21911135 \\
\hline $\mathrm{C}$ & -2.82807252 & 1.12401997 & 0.03629807 \\
\hline $\mathrm{C}$ & -2.92053256 & -1.65773980 & 0.02074187 \\
\hline $\mathrm{C}$ & -1.96885748 & -1.86416530 & -2.21118399 \\
\hline $\mathrm{C}$ & -1.33212246 & -2.85983292 & -1.22160354 \\
\hline $\mathrm{C}$ & -0.94114753 & -1.10417073 & -3.14253294 \\
\hline $\mathrm{C}$ & 2.98722916 & -1.49796450 & -1.15897098 \\
\hline $\mathrm{C}$ & 2.89756388 & 0.85846225 & -1.10912722 \\
\hline $\mathrm{F}$ & -2.87135847 & -2.50650277 & -3.04363766 \\
\hline $\mathrm{F}$ & -1.32907460 & -1.38898941 & -4.44042524 \\
\hline $\mathrm{F}$ & 1.35221179 & -3.85099490 & -3.09812700 \\
\hline $\mathrm{F}$ & 1.00910579 & -1.84100012 & -4.34435255 \\
\hline $\mathrm{F}$ & -2.94957033 & 1.77617473 & -3.16384901 \\
\hline $\mathrm{F}$ & -1.01011847 & 0.96852966 & -4.37679412 \\
\hline
\end{tabular}




\begin{tabular}{|l|l|l|l|}
\hline $\mathrm{F}$ & 0.04139312 & 4.17297395 & -2.25339498 \\
\hline $\mathrm{F}$ & -2.31966210 & 3.70821288 & -1.88152272 \\
\hline $\mathrm{F}$ & -0.45839163 & 4.87220656 & 1.75492692 \\
\hline $\mathrm{F}$ & 0.97881340 & 4.94319052 & -0.18032395 \\
\hline
\end{tabular}

\begin{tabular}{|c|c|c|c|}
\hline 'bis'- $\mathrm{C}_{60} \mathrm{~F}_{10}$ & & & \\
\hline $\mathrm{C}$ & 3.24933834 & 1.45316303 & 1.38687337 \\
\hline $\mathrm{C}$ & 3.02298103 & 0.73114937 & 2.63194289 \\
\hline $\mathrm{C}$ & 3.28244254 & -1.38303136 & 1.37243450 \\
\hline $\mathrm{C}$ & 3.02442895 & -0.65626373 & 2.61538313 \\
\hline $\bar{C}$ & 1.05269384 & 0.76511655 & 4.11414379 \\
\hline $\bar{C}$ & 2.00953104 & -1.38580798 & 3.37365020 \\
\hline $\mathrm{C}$ & 2.01264672 & 1.46222945 & 3.40104142 \\
\hline $\mathrm{C}$ & 1.05311657 & -0.69904794 & 4.10386755 \\
\hline $\mathrm{C}$ & 1.62755360 & -2.55851616 & 2.58853194 \\
\hline$\overline{\mathrm{C}}$ & 1.63003733 & 2.64901559 & 2.63168376 \\
\hline $\bar{C}$ & -1.20037194 & 0.03301135 & 4.09952881 \\
\hline $\mathrm{C}$ & -0.33952996 & 1.21841946 & 4.10360680 \\
\hline $\mathrm{C}$ & 0.31135033 & -2.98769903 & 2.59094415 \\
\hline $\mathrm{C}$ & 2.39515850 & -2.56129384 & 1.34658319 \\
\hline $\mathrm{C}$ & -0.29289399 & -3.39734037 & 1.33212316 \\
\hline $\mathrm{C}$ & -0.70246026 & -2.27057466 & 3.36804115 \\
\hline $\mathrm{C}$ & -1.94142587 & -2.26482202 & 2.58802299 \\
\hline $\mathrm{C}$ & -0.34043304 & -1.15099496 & 4.09968679 \\
\hline $\mathrm{C}$ & -1.92991889 & 2.33959634 & 2.58787298 \\
\hline $\bar{C}$ & 0.31112166 & 3.07954818 & 2.61487823 \\
\hline
\end{tabular}




\begin{tabular}{|c|c|c|c|}
\hline $\mathrm{C}$ & -1.67986351 & -2.96400072 & 1.32794451 \\
\hline $\mathrm{C}$ & -2.75460448 & -1.14519741 & 2.58787318 \\
\hline $\mathrm{C}$ & -3.33842568 & -0.68041039 & 1.32763920 \\
\hline $\mathrm{C}$ & -2.37693337 & 0.03492333 & 3.36773264 \\
\hline $\mathrm{C}$ & -2.74518921 & 1.22070208 & 2.59037943 \\
\hline $\mathrm{C}$ & -0.30003507 & 3.54950219 & 1.37177403 \\
\hline $\mathrm{C}$ & 2.38681793 & 2.64071826 & 1.38671352 \\
\hline $\mathrm{C}$ & -0.69667203 & 2.34023083 & 3.37314660 \\
\hline $\mathrm{C}$ & -1.69496455 & 3.07019832 & 1.34582812 \\
\hline $\mathrm{C}$ & -3.32149218 & 0.77258214 & 1.33156657 \\
\hline $\mathrm{C}$ & 2.28647581 & -2.56850873 & -1.23547159 \\
\hline $\mathrm{C}$ & -2.22326439 & -2.51154849 & 0.13859774 \\
\hline $\mathrm{C}$ & 1.76760486 & 3.06067745 & 0.21918409 \\
\hline $\mathrm{C}$ & 2.36262347 & -0.03164191 & -1.72229429 \\
\hline $\mathrm{C}$ & -0.17071830 & -2.91473545 & -1.08968376 \\
\hline $\mathrm{C}$ & 3.52315544 & -0.71469733 & 0.20515417 \\
\hline $\mathrm{C}$ & 0.43802426 & -3.33287643 & 0.15242548 \\
\hline $\mathrm{C}$ & 0.87413762 & -2.56966778 & -2.12324775 \\
\hline $\mathrm{C}$ & 1.82407752 & -2.95542169 & 0.16354470 \\
\hline $\mathrm{C}$ & 3.45730936 & 0.73421174 & 0.21949434 \\
\hline $\mathrm{C}$ & 0.41012583 & 3.57148132 & 0.20458121 \\
\hline $\mathrm{C}$ & -0.19783067 & 3.38547535 & -1.21690309 \\
\hline $\mathrm{C}$ & -2.24626313 & 2.64882574 & 0.16279738 \\
\hline $\mathrm{C}$ & -1.73512359 & 2.96861312 & -1.23621598 \\
\hline $\mathrm{C}$ & 0.31905539 & 1.11553046 & -2.36202411 \\
\hline $\mathrm{C}$ & -2.17296925 & 1.62576821 & -2.12379258 \\
\hline $\mathrm{C}$ & 0.70095208 & 2.25624418 & -1.72260643 \\
\hline
\end{tabular}




\begin{tabular}{|c|c|c|c|}
\hline $\mathrm{C}$ & -1.04353093 & 0.84562635 & -2.93022445 \\
\hline $\mathrm{C}$ & -2.82444935 & 0.73906066 & -1.09016861 \\
\hline $\mathrm{C}$ & 1.93667178 & 2.26087032 & -0.98431422 \\
\hline $\mathrm{C}$ & 0.48171329 & -1.25442807 & -2.92994812 \\
\hline $\mathrm{C}$ & 1.15979962 & -0.04206798 & -2.36185159 \\
\hline $\mathrm{C}$ & -2.84574240 & -0.61667554 & -1.10260682 \\
\hline $\mathrm{C}$ & -3.03394826 & 1.44750637 & 0.15178760 \\
\hline $\mathrm{C}$ & -3.07609671 & -1.33732269 & 0.13844012 \\
\hline $\mathrm{C}$ & -2.12032388 & -1.54026488 & -2.09491603 \\
\hline $\mathrm{C}$ & -1.46655062 & -2.51562447 & -1.10235132 \\
\hline $\mathrm{C}$ & -1.08965787 & -0.79183793 & -3.02797341 \\
\hline $\mathrm{C}$ & 3.15839939 & -1.23555258 & -1.21628245 \\
\hline $\mathrm{C}$ & 2.74917145 & 1.14217456 & -0.98416089 \\
\hline $\mathrm{F}$ & -3.02004539 & -2.19383329 & -2.92126822 \\
\hline $\mathrm{F}$ & -1.47619327 & -1.07272370 & -4.32626273 \\
\hline $\mathrm{F}$ & 1.02862031 & -3.57839131 & -3.05573373 \\
\hline $\bar{F}$ & 0.87493396 & -1.48093891 & -4.24172631 \\
\hline $\mathrm{F}$ & -3.08432967 & 2.08453643 & -3.05646469 \\
\hline $\mathrm{F}$ & -1.13717274 & 1.28935285 & -4.24209354 \\
\hline $\mathrm{F}$ & -0.07008478 & 4.53741582 & -1.96859931 \\
\hline $\mathrm{F}$ & -2.42888849 & 4.02355636 & -1.79733739 \\
\hline $\mathrm{F}$ & 3.07514120 & -3.55466842 & -1.79633213 \\
\hline $\mathrm{F}$ & 4.29346409 & -1.47050570 & -1.96778723 \\
\hline
\end{tabular}

\begin{tabular}{|l|l|l|l|}
\hline$' T '-\mathrm{C}_{60} \mathrm{~F}_{12}$ & & & \\
\hline $\mathrm{C}$ & -3.108323 & -1.777579 & -1.645466 \\
\hline
\end{tabular}




\begin{tabular}{|c|c|c|c|}
\hline $\mathrm{C}$ & -2.436741 & -3.040422 & -1.340662 \\
\hline $\mathrm{C}$ & -2.40831 & -3.516523 & -0.039327 \\
\hline $\mathrm{C}$ & -3.035178 & -2.733566 & 1.017118 \\
\hline $\mathrm{C}$ & -3.642571 & -1.524247 & 0.701112 \\
\hline $\mathrm{C}$ & -3.718373 & -1.04474 & -0.663391 \\
\hline $\mathrm{C}$ & -2.289675 & -1.072499 & -2.648881 \\
\hline $\mathrm{C}$ & -1.142883 & -1.92652 & -2.964852 \\
\hline $\mathrm{C}$ & -1.23503 & -3.142498 & -2.170052 \\
\hline $\mathrm{C}$ & -0.072782 & -3.70873 & -1.667135 \\
\hline $\mathrm{C}$ & -1.184568 & -4.115682 & 0.500391 \\
\hline $\mathrm{C}$ & -2.213767 & -2.859962 & 2.223656 \\
\hline $\mathrm{C}$ & -2.051261 & -1.772233 & 3.064745 \\
\hline $\mathrm{C}$ & -2.685529 & -0.498925 & 2.718938 \\
\hline $\mathrm{C}$ & -3.436422 & -0.371783 & 1.564917 \\
\hline $\mathrm{C}$ & -3.359239 & 0.824071 & 0.741206 \\
\hline $\mathrm{C}$ & -3.889173 & 0.501752 & -0.659046 \\
\hline $\mathrm{C}$ & -3.058489 & 1.253488 & -1.797019 \\
\hline $\mathrm{C}$ & -2.153665 & 0.286555 & -2.552321 \\
\hline $\mathrm{C}$ & 0.108948 & -1.375273 & -3.198501 \\
\hline $\mathrm{C}$ & 0.259555 & 0.052696 & -3.015377 \\
\hline $\mathrm{C}$ & -0.833017 & 0.836048 & -2.675566 \\
\hline $\mathrm{C}$ & -0.671127 & 1.945944 & -1.790189 \\
\hline $\mathrm{C}$ & -2.012717 & 2.475358 & -1.3446 \\
\hline $\mathrm{C}$ & -1.957798 & 2.913925 & 0.169962 \\
\hline $\mathrm{C}$ & -2.49201 & 1.819591 & 1.068361 \\
\hline $\mathrm{C}$ & -1.648486 & 1.669271 & 2.22707 \\
\hline $\mathrm{C}$ & -1.766355 & 0.571239 & 3.064379 \\
\hline
\end{tabular}




\begin{tabular}{|c|c|c|c|}
\hline $\mathrm{C}$ & -1.068959 & -3.713804 & 1.90473 \\
\hline $\mathrm{C}$ & 1.7846 & -0.284042 & 3.017686 \\
\hline $\mathrm{C}$ & 1.636653 & -1.660228 & 3.022052 \\
\hline $\mathrm{C}$ & 2.255176 & -2.424091 & 1.951697 \\
\hline $\mathrm{C}$ & 2.958278 & -1.74964 & 0.961366 \\
\hline $\mathrm{C}$ & 3.151248 & -0.318034 & 1.014873 \\
\hline $\mathrm{C}$ & 2.175194 & 1.883494 & 1.970193 \\
\hline $\mathrm{C}$ & 0.748435 & 1.769366 & 2.531221 \\
\hline $\mathrm{C}$ & 0.649683 & 0.562095 & 3.33564 \\
\hline $\mathrm{C}$ & -0.569603 & -0.017541 & 3.639431 \\
\hline $\mathrm{C}$ & -0.736883 & -1.47288 & 3.636086 \\
\hline $\mathrm{C}$ & 0.346272 & -2.279322 & 3.332892 \\
\hline $\mathrm{C}$ & 1.369317 & -3.524065 & 1.601535 \\
\hline $\mathrm{C}$ & 1.261374 & -3.894814 & 0.271622 \\
\hline $\mathrm{C}$ & 2.05871 & -3.212709 & -0.743169 \\
\hline $\mathrm{C}$ & 2.899284 & -2.181402 & -0.407119 \\
\hline $\mathrm{C}$ & 3.399913 & -1.084041 & -1.337366 \\
\hline $\mathrm{C}$ & 3.60408 & 0.224357 & -0.318749 \\
\hline $\mathrm{C}$ & 2.860339 & 1.588584 & -0.670818 \\
\hline $\mathrm{C}$ & 2.238188 & 2.521294 & 0.527755 \\
\hline $\mathrm{C}$ & -0.376884 & 2.310511 & 1.999923 \\
\hline $\mathrm{C}$ & -0.450609 & 3.256434 & 0.827634 \\
\hline $\mathrm{C}$ & 0.833861 & 3.104771 & -0.095822 \\
\hline $\mathrm{C}$ & 0.552754 & 2.165257 & -1.229377 \\
\hline $\mathrm{C}$ & 1.674403 & 1.341222 & -1.555095 \\
\hline $\mathrm{C}$ & 1.554395 & 0.327183 & -2.455649 \\
\hline $\mathrm{C}$ & 2.44355 & -0.908332 & -2.598716 \\
\hline
\end{tabular}




\begin{tabular}{|l|l|l|l|}
\hline C & 1.336796 & -1.996764 & -2.737456 \\
\hline C & 1.233359 & -3.116022 & -1.961752 \\
\hline C & 0.175745 & -3.431584 & 2.445341 \\
\hline C & -0.051728 & -4.204656 & -0.291164 \\
\hline C & 2.584168 & 0.400804 & 2.015071 \\
\hline F & 2.981718 & 2.651082 & 2.794088 \\
\hline F & 3.081154 & 3.612806 & 0.625245 \\
\hline F & 3.80441 & 2.387197 & -1.300059 \\
\hline F & 4.959602 & 0.492154 & -0.334963 \\
\hline F & 1.139953 & 4.378731 & -0.550478 \\
\hline F & -0.517718 & 4.576985 & 1.223681 \\
\hline F & -2.683078 & 4.082427 & 0.294745 \\
\hline F & -2.403607 & 3.578656 & -2.080666 \\
\hline F & -5.215032 & 0.857475 & -0.813438 \\
\hline F & -3.979275 & 1.847129 & -2.6373 \\
\hline F & 4.659967 & -1.370632 & -1.825298 \\
\hline F & 3.241158 & -0.860785 & -3.724865 \\
\hline
\end{tabular}

\begin{tabular}{|l|l|l|l|}
\hline 'bis' $-\mathrm{C}_{60} \mathrm{~F}_{12}$ & & & \\
\hline $\mathrm{C}$ & 3.475117 & 1.060191 & -0.457245 \\
\hline $\mathrm{C}$ & 3.007904 & 2.43746 & -0.53195 \\
\hline $\mathrm{C}$ & 2.593827 & 3.121678 & 0.598351 \\
\hline $\mathrm{C}$ & 2.592911 & 2.426968 & 1.876583 \\
\hline $\mathrm{C}$ & 3.000113 & 1.103305 & 1.915624 \\
\hline $\mathrm{C}$ & 3.472798 & 0.407083 & 0.729774 \\
\hline $\mathrm{C}$ & 3.355981 & 0.397902 & -1.828476 \\
\hline
\end{tabular}




\begin{tabular}{|c|c|c|c|}
\hline $\mathrm{C}$ & 2.325037 & 1.354105 & -2.49446 \\
\hline $\mathrm{C}$ & 2.297246 & 2.620672 & -1.788946 \\
\hline $\mathrm{C}$ & 1.185018 & 3.452657 & -1.839694 \\
\hline $\mathrm{C}$ & 1.422389 & 3.99782 & 0.55312 \\
\hline $\bar{C}$ & 1.423507 & 2.872783 & 2.636761 \\
\hline $\mathrm{C}$ & 0.731858 & 1.964586 & 3.419764 \\
\hline $\mathrm{C}$ & 1.178007 & 0.570487 & 3.46768 \\
\hline $\mathrm{C}$ & 2.269747 & 0.149157 & 2.729858 \\
\hline $\mathrm{C}$ & 2.284625 & -1.135978 & 2.051475 \\
\hline $\mathrm{C}$ & 3.366095 & -1.113486 & 0.960977 \\
\hline $\mathrm{C}$ & 2.971328 & -1.950451 & -0.319528 \\
\hline $\mathrm{C}$ & 2.86521 & -1.133155 & -1.730106 \\
\hline $\mathrm{C}$ & 1.19017 & 0.95638 & -3.142496 \\
\hline $\mathrm{C}$ & 0.716026 & -0.416998 & -2.924598 \\
\hline $\mathrm{C}$ & 1.404266 & -1.261683 & -2.078658 \\
\hline $\mathrm{C}$ & 0.689191 & -2.134893 & -1.234087 \\
\hline $\mathrm{C}$ & 1.580653 & -2.836938 & -0.252631 \\
\hline $\mathrm{C}$ & 0.839278 & -3.042657 & 1.12927 \\
\hline $\mathrm{C}$ & 1.17995 & -1.924928 & 2.084801 \\
\hline $\mathrm{C}$ & -0.000027 & -1.464637 & 2.773284 \\
\hline $\mathrm{C}$ & -0.000026 & -0.278847 & 3.491111 \\
\hline $\mathrm{C}$ & 0.693439 & 3.837855 & 1.813013 \\
\hline $\mathrm{C}$ & -3.000117 & 1.10332 & 1.915601 \\
\hline $\mathrm{C}$ & -2.59291 & 2.426963 & 1.876565 \\
\hline $\mathrm{C}$ & -2.59381 & 3.121671 & 0.598332 \\
\hline $\mathrm{C}$ & -3.007887 & 2.437467 & -0.531964 \\
\hline $\mathrm{C}$ & -3.47511 & 1.060189 & -0.457282 \\
\hline
\end{tabular}




\begin{tabular}{|c|c|c|c|}
\hline $\mathrm{C}$ & -3.366137 & -1.11343 & 0.961023 \\
\hline $\mathrm{C}$ & -2.284661 & -1.135951 & 2.05145 \\
\hline $\mathrm{C}$ & -2.269764 & 0.149194 & 2.729826 \\
\hline $\mathrm{C}$ & -1.178047 & 0.570501 & 3.467656 \\
\hline $\bar{C}$ & -0.731899 & 1.964596 & 3.419768 \\
\hline $\mathrm{C}$ & -1.423535 & 2.872784 & 2.636759 \\
\hline $\mathrm{C}$ & -1.422383 & 3.997816 & 0.553108 \\
\hline $\mathrm{C}$ & -0.731848 & 4.15341 & -0.636243 \\
\hline $\mathrm{C}$ & -1.184992 & 3.452657 & -1.839697 \\
\hline $\mathrm{C}$ & -2.297213 & 2.620678 & -1.78895 \\
\hline $\mathrm{C}$ & -2.325044 & 1.354112 & -2.494486 \\
\hline $\mathrm{C}$ & -3.355999 & 0.397986 & -1.828541 \\
\hline $\mathrm{C}$ & -2.865202 & -1.13322 & -1.730164 \\
\hline $\mathrm{C}$ & -2.971304 & -1.950553 & -0.319536 \\
\hline $\mathrm{C}$ & -1.179996 & -1.924908 & 2.084775 \\
\hline $\mathrm{C}$ & -0.839321 & -3.042629 & 1.129247 \\
\hline $\mathrm{C}$ & -1.580566 & -2.837001 & -0.252636 \\
\hline $\mathrm{C}$ & -0.689165 & -2.134898 & -1.234101 \\
\hline $\mathrm{C}$ & -1.404274 & -1.261708 & -2.078693 \\
\hline $\mathrm{C}$ & -0.716035 & -0.417 & -2.924612 \\
\hline $\mathrm{C}$ & -1.190162 & 0.956378 & -3.142492 \\
\hline $\mathrm{C}$ & 0.000007 & 1.802449 & -3.21614 \\
\hline $\mathrm{C}$ & 0.000013 & 3.035403 & -2.582701 \\
\hline $\mathrm{C}$ & -0.693458 & 3.837848 & 1.813006 \\
\hline $\mathrm{C}$ & 0.731873 & 4.153419 & -0.636239 \\
\hline $\mathrm{C}$ & -3.472785 & 0.40708 & 0.729739 \\
\hline $\mathrm{F}$ & -4.565607 & -1.625904 & 1.428278 \\
\hline
\end{tabular}




\begin{tabular}{|l|l|l|l|}
\hline F & -3.983013 & -2.876739 & -0.496954 \\
\hline F & -3.662308 & -1.831805 & -2.624197 \\
\hline F & -4.551343 & 0.392911 & -2.519083 \\
\hline F & -1.938378 & -4.099225 & -0.702175 \\
\hline F & -1.205303 & -4.279647 & 1.620461 \\
\hline F & 1.20522 & -4.279677 & 1.620451 \\
\hline F & 1.938573 & -4.099131 & -0.702183 \\
\hline F & 3.98307 & -2.876636 & -0.497005 \\
\hline F & 4.565552 & -1.625938 & 1.428291 \\
\hline F & 3.662264 & -1.831871 & -2.624129 \\
\hline F & 4.551338 & 0.392957 & -2.519052 \\
\hline
\end{tabular}

\begin{tabular}{|l|l|l|l|}
\hline${ }^{\prime}{ }^{\prime}-\mathrm{C}_{60} \mathrm{~F}_{14}$ & & & \\
\hline $\mathrm{C}$ & -1.191779 & -3.424538 & -1.403139 \\
\hline $\mathrm{C}$ & -0.000043 & -3.963247 & -0.749977 \\
\hline $\mathrm{C}$ & -0.000041 & -4.164496 & 0.622256 \\
\hline $\mathrm{C}$ & -1.182147 & -3.808909 & 1.399406 \\
\hline $\mathrm{C}$ & -2.286329 & -3.272157 & 0.750535 \\
\hline $\mathrm{C}$ & -2.316367 & -3.108538 & -0.68938 \\
\hline $\mathrm{C}$ & -0.729669 & -2.491535 & -2.443721 \\
\hline $\mathrm{C}$ & 0.729644 & -2.491553 & -2.443733 \\
\hline $\mathrm{C}$ & 1.191692 & -3.424518 & -1.403107 \\
\hline $\mathrm{C}$ & 2.316305 & -3.108577 & -0.689369 \\
\hline $\mathrm{C}$ & 1.18208 & -3.808941 & 1.399405 \\
\hline $\mathrm{C}$ & -0.731686 & -3.254517 & 2.678569 \\
\hline $\mathrm{C}$ & -1.420637 & -2.201566 & 3.257464 \\
\hline
\end{tabular}




\begin{tabular}{|c|c|c|c|}
\hline $\mathrm{C}$ & -2.579761 & -1.638575 & 2.563585 \\
\hline $\mathrm{C}$ & -2.984725 & -2.140207 & 1.340183 \\
\hline $\mathrm{C}$ & -3.435525 & -1.258681 & 0.275565 \\
\hline $\mathrm{C}$ & -3.351613 & -2.006165 & -1.05876 \\
\hline $\mathrm{C}$ & -2.884494 & -1.046838 & -2.245433 \\
\hline $\mathrm{C}$ & -1.442373 & -1.335008 & -2.643986 \\
\hline $\mathrm{C}$ & 1.442377 & -1.335026 & -2.643973 \\
\hline $\mathrm{C}$ & 0.695598 & -0.122067 & -2.719943 \\
\hline $\mathrm{C}$ & -0.695599 & -0.122044 & -2.71998 \\
\hline $\mathrm{C}$ & -1.417682 & 0.95797 & -2.12861 \\
\hline $\mathrm{C}$ & -2.87746 & 0.60691 & -1.97915 \\
\hline $\mathrm{C}$ & -3.4318 & 1.164751 & -0.612328 \\
\hline $\mathrm{C}$ & -3.410348 & 0.090195 & 0.452874 \\
\hline $\mathrm{C}$ & -2.920619 & 0.63109 & 1.695627 \\
\hline $\mathrm{C}$ & -2.559487 & -0.197585 & 2.74588 \\
\hline $\mathrm{C}$ & 0.731628 & -3.254541 & 2.678583 \\
\hline $\mathrm{C}$ & 0.725353 & 1.313721 & 3.260065 \\
\hline $\mathrm{C}$ & 1.407904 & 0.147704 & 3.561034 \\
\hline $\mathrm{C}$ & 2.559456 & -0.197636 & 2.745908 \\
\hline $\mathrm{C}$ & 2.92061 & 0.631032 & 1.695645 \\
\hline $\mathrm{C}$ & 2.250956 & 1.88706 & 1.461513 \\
\hline $\mathrm{C}$ & 0.000028 & 3.123809 & 1.797055 \\
\hline $\mathrm{C}$ & -1.173663 & 2.221584 & 2.216152 \\
\hline $\mathrm{C}$ & -0.725356 & 1.313733 & 3.26006 \\
\hline $\mathrm{C}$ & -1.407935 & 0.147731 & 3.561009 \\
\hline $\mathrm{C}$ & -0.692124 & -1.091279 & 3.874948 \\
\hline $\mathrm{C}$ & 0.692063 & -1.091293 & 3.87496 \\
\hline
\end{tabular}




\begin{tabular}{|c|c|c|c|}
\hline $\mathrm{C}$ & 2.579715 & -1.638631 & 2.563627 \\
\hline $\mathrm{C}$ & 2.984638 & -2.140238 & 1.340178 \\
\hline $\mathrm{C}$ & 3.435576 & -1.25873 & 0.275572 \\
\hline $\mathrm{C}$ & 3.410453 & 0.090153 & 0.452912 \\
\hline $\bar{C}$ & 3.431866 & 1.164699 & -0.612298 \\
\hline $\mathrm{C}$ & 2.598754 & 2.440608 & 0.102916 \\
\hline $\mathrm{C}$ & 1.297781 & 3.001372 & -0.619861 \\
\hline $\mathrm{C}$ & 0.000038 & 3.491181 & 0.262349 \\
\hline $\mathrm{C}$ & -2.250912 & 1.887087 & 1.4615 \\
\hline $\mathrm{C}$ & -2.598719 & 2.440657 & 0.102933 \\
\hline $\mathrm{C}$ & -1.297736 & 3.001409 & -0.619851 \\
\hline $\mathrm{C}$ & -0.714645 & 1.948679 & -1.511894 \\
\hline $\mathrm{C}$ & 0.714637 & 1.948685 & -1.511913 \\
\hline $\mathrm{C}$ & 1.417694 & 0.957934 & -2.128548 \\
\hline $\mathrm{C}$ & 2.877477 & 0.606852 & -1.979108 \\
\hline $\mathrm{C}$ & 2.884505 & -1.04688 & -2.245415 \\
\hline $\mathrm{C}$ & 3.35158 & -2.006231 & -1.058761 \\
\hline $\mathrm{C}$ & 1.420585 & -2.201604 & 3.257506 \\
\hline $\mathrm{C}$ & 2.286295 & -3.272244 & 0.750546 \\
\hline $\mathrm{C}$ & 1.173696 & 2.221559 & 2.216151 \\
\hline $\bar{F}$ & 0.00004 & 4.313374 & 2.505814 \\
\hline $\mathrm{F}$ & 0.000032 & 4.871117 & 0.191968 \\
\hline $\mathrm{F}$ & 1.697725 & 4.120754 & -1.333005 \\
\hline $\mathrm{F}$ & 3.509096 & 3.474885 & 0.17287 \\
\hline $\mathrm{F}$ & -1.697626 & 4.120806 & -1.333011 \\
\hline $\mathrm{F}$ & -3.509081 & 3.474909 & 0.172899 \\
\hline $\mathrm{F}$ & -4.706572 & 1.64449 & -0.836971 \\
\hline
\end{tabular}




\begin{tabular}{|l|l|l|l|}
\hline F & -3.649588 & 1.140347 & -2.993227 \\
\hline F & -4.561366 & -2.561759 & -1.427045 \\
\hline F & -3.756149 & -1.248751 & -3.295607 \\
\hline F & 4.70661 & 1.644463 & -0.836974 \\
\hline F & 3.649584 & 1.140301 & -2.9932 \\
\hline F & 3.756171 & -1.248788 & -3.295604 \\
\hline F & 4.561316 & -2.561868 & -1.427062 \\
\hline
\end{tabular}

\begin{tabular}{|l|l|l|l|}
\hline${ }^{\prime}$ bis' $-\mathrm{C}_{60} \mathrm{~F}_{14}$ & & & \\
\hline $\mathrm{C}$ & -3.439554 & -0.365455 & 0.429618 \\
\hline $\mathrm{C}$ & -3.208492 & -1.791007 & 0.487887 \\
\hline $\mathrm{C}$ & -2.730759 & -2.400081 & 1.641397 \\
\hline $\mathrm{C}$ & -2.383484 & -1.572148 & 2.784234 \\
\hline $\mathrm{C}$ & -2.55637 & -0.202857 & 2.68207 \\
\hline $\mathrm{C}$ & -3.125732 & 0.41326 & 1.495212 \\
\hline $\mathrm{C}$ & -3.592269 & 0.105342 & -0.995865 \\
\hline $\mathrm{C}$ & -3.133061 & -1.238877 & -1.881692 \\
\hline $\mathrm{C}$ & -2.836711 & -2.279568 & -0.810922 \\
\hline $\mathrm{C}$ & -1.924983 & -3.300591 & -0.903461 \\
\hline $\mathrm{C}$ & -1.766801 & -3.486081 & 1.550039 \\
\hline $\mathrm{C}$ & -1.181757 & -2.135653 & 3.403271 \\
\hline $\mathrm{C}$ & -0.212834 & -1.28165 & 3.901462 \\
\hline $\mathrm{C}$ & -0.40969 & 0.166891 & 3.802831 \\
\hline $\mathrm{C}$ & -1.5425 & 0.692975 & 3.207064 \\
\hline $\mathrm{C}$ & -1.485568 & 1.864273 & 2.348052 \\
\hline $\mathrm{C}$ & -2.751449 & 1.906083 & 1.475104 \\
\hline
\end{tabular}




\begin{tabular}{|c|c|c|c|}
\hline $\mathrm{C}$ & -2.498648 & 2.479324 & 0.027867 \\
\hline $\mathrm{C}$ & -2.81079 & 1.471371 & -1.231739 \\
\hline $\mathrm{C}$ & -1.913364 & -1.089835 & -2.897418 \\
\hline $\mathrm{C}$ & -1.09011 & 0.156104 & -2.587072 \\
\hline $\bar{C}$ & -1.442219 & 1.203803 & -1.782179 \\
\hline $\mathrm{C}$ & -0.442718 & 2.045329 & -1.238578 \\
\hline $\mathrm{C}$ & -0.993812 & 3.063514 & -0.287861 \\
\hline $\mathrm{C}$ & 0.032698 & 3.321135 & 0.891318 \\
\hline $\mathrm{C}$ & -0.282273 & 2.425584 & 2.063807 \\
\hline $\mathrm{C}$ & 0.920731 & 1.838754 & 2.600338 \\
\hline $\mathrm{C}$ & 0.871554 & 0.773907 & 3.486521 \\
\hline $\mathrm{C}$ & -0.793594 & -3.318447 & 2.631954 \\
\hline $\mathrm{C}$ & 3.237498 & -1.341036 & 1.659511 \\
\hline $\mathrm{C}$ & 2.62325 & -2.558842 & 1.904179 \\
\hline $\mathrm{C}$ & 2.263671 & -3.400656 & 0.773809 \\
\hline $\mathrm{C}$ & 2.557349 & -2.962925 & -0.505691 \\
\hline $\mathrm{C}$ & 3.242298 & -1.698456 & -0.734395 \\
\hline $\mathrm{C}$ & 3.75909 & 0.625247 & 0.312361 \\
\hline $\mathrm{C}$ & 2.931679 & 0.991949 & 1.55411 \\
\hline $\mathrm{C}$ & 2.844695 & -0.170244 & 2.422011 \\
\hline $\mathrm{C}$ & 1.867643 & -0.279558 & 3.395134 \\
\hline $\mathrm{C}$ & 1.202046 & -1.558954 & 3.648745 \\
\hline $\mathrm{C}$ & 1.569874 & -2.674029 & 2.914455 \\
\hline $\mathrm{C}$ & 0.97657 & -4.034531 & 1.067756 \\
\hline $\mathrm{C}$ & 0.052187 & -4.199872 & 0.051669 \\
\hline $\mathrm{C}$ & 0.366217 & -3.764393 & -1.309336 \\
\hline $\mathrm{C}$ & 1.595452 & -3.170095 & -1.581205 \\
\hline
\end{tabular}




\begin{tabular}{|c|c|c|c|}
\hline $\mathrm{C}$ & 1.681229 & -2.039061 & -2.471644 \\
\hline $\mathrm{C}$ & 2.959429 & -1.208948 & -2.153662 \\
\hline $\mathrm{C}$ & 2.73575 & 0.382403 & -2.211948 \\
\hline $\mathrm{C}$ & 3.255887 & 1.346818 & -0.998556 \\
\hline $\mathrm{C}$ & 1.998918 & 1.974533 & 1.652835 \\
\hline $\mathrm{C}$ & 1.658954 & 3.003581 & 0.60241 \\
\hline $\mathrm{C}$ & 2.060642 & 2.474431 & -0.831931 \\
\hline $\mathrm{C}$ & 0.887552 & 1.806172 & -1.484406 \\
\hline $\mathrm{C}$ & 1.268722 & 0.710187 & -2.282591 \\
\hline $\mathrm{C}$ & 0.294072 & -0.092616 & -2.830975 \\
\hline $\mathrm{C}$ & 0.504581 & -1.533297 & -2.9642 \\
\hline $\mathrm{C}$ & -0.786948 & -2.154306 & -2.747569 \\
\hline $\mathrm{C}$ & -0.849838 & -3.236404 & -1.912728 \\
\hline $\mathrm{C}$ & 0.546398 & -3.581188 & 2.393105 \\
\hline $\mathrm{C}$ & -1.359705 & -3.911454 & 0.296137 \\
\hline $\mathrm{C}$ & 3.574254 & -0.905949 & 0.314056 \\
\hline $\mathrm{F}$ & 5.09314 & 0.960596 & 0.477077 \\
\hline $\mathrm{F}$ & 4.342194 & 2.037399 & -1.501795 \\
\hline $\mathrm{F}$ & 3.412037 & 0.811661 & -3.343212 \\
\hline $\bar{F}$ & 3.980616 & -1.508143 & -3.032353 \\
\hline $\mathrm{F}$ & 2.497116 & 3.572045 & -1.556943 \\
\hline $\mathrm{F}$ & 2.309218 & 4.202394 & 0.809235 \\
\hline $\mathrm{F}$ & -0.027861 & 4.659844 & 1.217271 \\
\hline $\mathrm{F}$ & -1.200938 & 4.28669 & -0.907315 \\
\hline $\mathrm{F}$ & -3.357114 & 3.550401 & -0.125844 \\
\hline $\bar{F}$ & -3.741435 & 2.679302 & 2.058143 \\
\hline $\mathrm{F}$ & -3.583018 & 2.213627 & -2.111939 \\
\hline
\end{tabular}




\begin{tabular}{|l|l|l|l|}
\hline $\mathrm{F}$ & -4.912663 & 0.331018 & -1.331762 \\
\hline $\mathrm{F}$ & -4.241367 & -1.580454 & -2.630748 \\
\hline $\mathrm{F}$ & -2.43316 & -1.08565 & -4.176282 \\
\hline
\end{tabular}

\begin{tabular}{|c|c|c|c|}
\hline${ }^{\prime} T '-\mathrm{C}_{60} \mathrm{~F}_{16}$ & & & \\
\hline $\bar{C}$ & -0.04878 & -3.737334 & -0.495495 \\
\hline $\mathrm{C}$ & 1.106121 & -3.674099 & 0.384382 \\
\hline $\mathrm{C}$ & 0.930226 & -3.591202 & 1.756905 \\
\hline $\mathrm{C}$ & -0.413229 & -3.486727 & 2.308326 \\
\hline $\mathrm{C}$ & -1.494036 & -3.479418 & 1.437268 \\
\hline $\bar{C}$ & -1.320765 & -3.64916 & 0.009441 \\
\hline $\mathrm{C}$ & 0.309601 & -3.020416 & -1.7207 \\
\hline $\mathrm{C}$ & 1.85216 & -2.777493 & -1.751559 \\
\hline $\mathrm{C}$ & 2.181847 & -2.94891 & -0.274561 \\
\hline $\mathrm{C}$ & 3.007075 & -2.146717 & 0.442027 \\
\hline $\mathrm{C}$ & 1.812682 & -2.734688 & 2.536321 \\
\hline $\mathrm{C}$ & -0.362172 & -2.565181 & 3.446693 \\
\hline $\mathrm{C}$ & -1.407139 & -1.682915 & 3.665666 \\
\hline $\mathrm{C}$ & -2.5389 & -1.673144 & 2.736824 \\
\hline $\mathrm{C}$ & -2.573118 & -2.526604 & 1.648801 \\
\hline $\mathrm{C}$ & -3.06054 & -2.081525 & 0.353834 \\
\hline $\mathrm{C}$ & -2.542556 & -3.037067 & -0.728252 \\
\hline $\mathrm{C}$ & -2.146222 & -2.267034 & -2.064729 \\
\hline $\mathrm{C}$ & -0.628407 & -2.201081 & -2.240448 \\
\hline $\mathrm{C}$ & 2.280353 & -1.327413 & -2.290295 \\
\hline $\mathrm{C}$ & 1.082051 & -0.422999 & -2.325079 \\
\hline
\end{tabular}




\begin{tabular}{|c|c|c|c|}
\hline $\mathrm{C}$ & -0.2248 & -0.835574 & -2.486387 \\
\hline $\mathrm{C}$ & -1.305546 & 0.046797 & -2.257886 \\
\hline $\mathrm{C}$ & -2.624988 & -0.676098 & -2.220151 \\
\hline $\mathrm{C}$ & -3.558792 & -0.031404 & -1.123391 \\
\hline $\mathrm{C}$ & -3.438305 & -0.785657 & 0.181554 \\
\hline $\mathrm{C}$ & -3.339557 & 0.135457 & 1.285642 \\
\hline $\mathrm{C}$ & -2.950584 & -0.293516 & 2.544616 \\
\hline $\mathrm{C}$ & 1.0193 & -2.104394 & 3.593227 \\
\hline $\mathrm{C}$ & -0.356676 & 2.243391 & 2.990275 \\
\hline $\mathrm{C}$ & 0.559818 & 1.443106 & 3.650212 \\
\hline $\mathrm{C}$ & 1.889163 & 1.314976 & 3.078654 \\
\hline $\mathrm{C}$ & 2.189999 & 1.978218 & 1.899393 \\
\hline $\mathrm{C}$ & 1.246952 & 2.878148 & 1.281661 \\
\hline $\mathrm{C}$ & -1.287148 & 3.378149 & 1.045168 \\
\hline $\mathrm{C}$ & -2.218461 & 2.264068 & 1.555957 \\
\hline $\mathrm{C}$ & -1.725736 & 1.787569 & 2.838067 \\
\hline $\mathrm{C}$ & -2.099916 & 0.559775 & 3.355895 \\
\hline $\mathrm{C}$ & -1.134035 & -0.296272 & 4.048768 \\
\hline $\mathrm{C}$ & 0.173047 & 0.13771 & 4.191516 \\
\hline $\mathrm{C}$ & 2.338642 & -0.054011 & 3.265179 \\
\hline $\mathrm{C}$ & 3.06732 & -0.645365 & 2.248586 \\
\hline $\mathrm{C}$ & 3.443603 & 0.088568 & 1.052503 \\
\hline $\mathrm{C}$ & 3.025479 & 1.37158 & 0.892929 \\
\hline $\mathrm{C}$ & 2.932387 & 2.133741 & -0.406397 \\
\hline $\mathrm{C}$ & 1.6612 & 3.204347 & -0.131579 \\
\hline $\mathrm{C}$ & 0.405704 & 3.129693 & -1.097452 \\
\hline $\mathrm{C}$ & -1.114236 & 3.385416 & -0.521898 \\
\hline
\end{tabular}




\begin{tabular}{|c|c|c|c|}
\hline $\mathrm{C}$ & -3.012969 & 1.456571 & 0.807268 \\
\hline $\mathrm{C}$ & -3.253521 & 1.567232 & -0.676706 \\
\hline $\mathrm{C}$ & -2.044552 & 2.326555 & -1.372179 \\
\hline $\mathrm{C}$ & -1.035445 & 1.327681 & -1.84821 \\
\hline $\mathrm{C}$ & 0.298447 & 1.75447 & -1.680009 \\
\hline $\mathrm{C}$ & 1.344727 & 0.894275 & -1.919186 \\
\hline $\mathrm{C}$ & 2.794192 & 1.125411 & -1.616178 \\
\hline $\mathrm{C}$ & 3.379824 & -0.415935 & -1.49331 \\
\hline $\mathrm{C}$ & 3.793594 & -0.916534 & -0.056753 \\
\hline $\mathrm{C}$ & 1.278596 & -0.792182 & 3.954273 \\
\hline $\mathrm{C}$ & 2.799276 & -2.02221 & 1.875978 \\
\hline $\mathrm{C}$ & -0.000607 & 3.003723 & 1.803353 \\
\hline $\mathrm{F}$ & -1.746301 & 4.630076 & 1.417544 \\
\hline $\bar{F}$ & -1.478699 & 4.643543 & -0.956768 \\
\hline $\bar{F}$ & 0.594869 & 4.095368 & -2.073314 \\
\hline $\mathrm{F}$ & 2.210262 & 4.463487 & -0.247452 \\
\hline $\mathrm{F}$ & -2.583348 & 3.064557 & -2.413169 \\
\hline $\mathrm{F}$ & -4.409136 & 2.255748 & -0.978321 \\
\hline $\mathrm{F}$ & -4.849452 & -0.03103 & -1.610084 \\
\hline $\mathrm{F}$ & -3.295134 & -0.612778 & -3.427311 \\
\hline $\bar{F}$ & -2.73907 & -2.940777 & -3.113151 \\
\hline $\mathrm{F}$ & -3.470702 & -4.00349 & -1.06199 \\
\hline $\mathrm{F}$ & 2.47421 & -3.71357 & -2.552595 \\
\hline $\mathrm{F}$ & 2.813069 & -1.535402 & -3.552319 \\
\hline $\mathrm{F}$ & 4.526305 & -0.436987 & -2.263158 \\
\hline $\mathrm{F}$ & 5.153049 & -1.180612 & -0.088777 \\
\hline $\mathrm{F}$ & 3.468877 & 1.684308 & -2.688863 \\
\hline
\end{tabular}




\begin{tabular}{|l|l|l|l|}
\hline $\mathrm{F}$ & 4.036768 & 2.926659 & -0.635851 \\
\hline
\end{tabular}

\begin{tabular}{|c|c|c|c|}
\hline 'bis'- $\mathrm{C}_{60} \mathrm{~F}_{16}$ & & & \\
\hline $\mathrm{C}$ & -3.449818 & -0.886648 & 0.338178 \\
\hline $\mathrm{C}$ & -2.966356 & -2.103271 & 0.950807 \\
\hline $\mathrm{C}$ & -2.576205 & -2.13506 & 2.283853 \\
\hline $\mathrm{C}$ & -2.587129 & -0.902809 & 3.054036 \\
\hline $\mathrm{C}$ & -2.993844 & 0.261695 & 2.426173 \\
\hline $\mathrm{C}$ & -3.462654 & 0.268988 & 1.050566 \\
\hline $\mathrm{C}$ & -3.442387 & -0.998001 & -1.167436 \\
\hline $\mathrm{C}$ & -2.598236 & -2.423277 & -1.437323 \\
\hline $\mathrm{C}$ & -2.295679 & -2.909044 & -0.031755 \\
\hline $\mathrm{C}$ & -1.189956 & -3.62918 & 0.33675 \\
\hline $\mathrm{C}$ & -1.418381 & -2.917659 & 2.687214 \\
\hline $\mathrm{C}$ & -1.423072 & -0.910875 & 3.942613 \\
\hline $\bar{C}$ & -0.731552 & 0.268064 & 4.166356 \\
\hline $\mathrm{C}$ & -1.177642 & 1.499618 & 3.510536 \\
\hline $\mathrm{C}$ & -2.26964 & 1.497147 & 2.660533 \\
\hline $\mathrm{C}$ & -2.287358 & 2.273337 & 1.431851 \\
\hline $\mathrm{C}$ & -3.37287 & 1.704673 & 0.500347 \\
\hline $\mathrm{C}$ & -2.985636 & 1.772568 & -1.025439 \\
\hline $\mathrm{C}$ & -2.872537 & 0.335 & -1.817231 \\
\hline $\mathrm{C}$ & -1.262327 & -2.359709 & -2.31254 \\
\hline $\mathrm{C}$ & -0.710612 & -0.946774 & -2.305059 \\
\hline $\mathrm{C}$ & -1.395292 & 0.202793 & -2.023148 \\
\hline $\mathrm{C}$ & -0.685049 & 1.39372 & -1.764982 \\
\hline
\end{tabular}




\begin{tabular}{|c|c|c|c|}
\hline $\mathrm{C}$ & -1.575355 & 2.538132 & -1.39208 \\
\hline $\mathrm{C}$ & -0.841563 & 3.44778 & -0.322076 \\
\hline $\mathrm{C}$ & -1.180932 & 2.973477 & 1.069118 \\
\hline $\mathrm{C}$ & 0.001093 & 2.917779 & 1.893833 \\
\hline $\mathrm{C}$ & 0.000897 & 2.247374 & 3.107191 \\
\hline $\mathrm{C}$ & -0.693157 & -2.158598 & 3.711194 \\
\hline $\mathrm{C}$ & 2.994282 & 0.259871 & 2.426031 \\
\hline $\mathrm{C}$ & 2.586805 & -0.904413 & 3.05379 \\
\hline $\mathrm{C}$ & 2.574949 & -2.136569 & 2.283489 \\
\hline $\mathrm{C}$ & 2.964978 & -2.104995 & 0.950375 \\
\hline $\mathrm{C}$ & 3.449225 & -0.888684 & 0.337879 \\
\hline $\mathrm{C}$ & 3.374162 & 1.702642 & 0.500131 \\
\hline $\mathrm{C}$ & 2.289109 & 2.271973 & 1.431865 \\
\hline $\mathrm{C}$ & 2.270931 & 1.495761 & 2.660476 \\
\hline $\mathrm{C}$ & 1.178948 & 1.498857 & 3.510483 \\
\hline $\mathrm{C}$ & 0.732117 & 0.267605 & 4.166327 \\
\hline $\mathrm{C}$ & 1.422844 & -0.91177 & 3.942493 \\
\hline $\mathrm{C}$ & 1.416704 & -2.918487 & 2.686998 \\
\hline $\mathrm{C}$ & 0.728621 & -3.631485 & 1.718924 \\
\hline $\mathrm{C}$ & 1.187529 & -3.629869 & 0.336619 \\
\hline $\mathrm{C}$ & 2.293649 & -2.910368 & -0.032032 \\
\hline $\mathrm{C}$ & 2.596091 & -2.425306 & -1.437688 \\
\hline $\mathrm{C}$ & 3.441764 & -0.999191 & -1.167691 \\
\hline $\mathrm{C}$ & 2.872701 & 0.333152 & -1.817308 \\
\hline $\mathrm{C}$ & 2.98665 & 1.770787 & -1.02555 \\
\hline $\mathrm{C}$ & 1.183123 & 2.972809 & 1.069123 \\
\hline $\mathrm{C}$ & 0.844131 & 3.447108 & -0.322238 \\
\hline
\end{tabular}




\begin{tabular}{|c|c|c|c|}
\hline $\mathrm{C}$ & 1.57673 & 2.537197 & -1.391971 \\
\hline $\mathrm{C}$ & 0.685809 & 1.393273 & -1.765082 \\
\hline $\mathrm{C}$ & 1.395289 & 0.201926 & -2.023285 \\
\hline $\mathrm{C}$ & 0.709927 & -0.947258 & -2.305122 \\
\hline $\mathrm{C}$ & 1.260526 & -2.360671 & -2.312728 \\
\hline $\mathrm{C}$ & -0.001183 & -3.057476 & -1.730309 \\
\hline $\mathrm{C}$ & -0.00128 & -3.672012 & -0.540213 \\
\hline $\mathrm{C}$ & 0.692099 & -2.159014 & 3.711108 \\
\hline $\mathrm{C}$ & -0.730887 & -3.631059 & 1.719017 \\
\hline $\mathrm{C}$ & 3.46288 & 0.266888 & 1.05042 \\
\hline $\bar{F}$ & 4.57504 & 2.374533 & 0.654442 \\
\hline $\mathrm{F}$ & 3.983814 & 2.480232 & -1.662576 \\
\hline $\mathrm{F}$ & 3.552711 & 0.515377 & -3.010514 \\
\hline $\mathrm{F}$ & 4.700896 & -1.213002 & -1.688437 \\
\hline $\mathrm{F}$ & 1.870164 & 3.347185 & -2.476941 \\
\hline $\mathrm{F}$ & 1.214182 & 4.75517 & -0.547003 \\
\hline $\mathrm{F}$ & -1.210654 & 4.756107 & -0.546594 \\
\hline $\mathrm{F}$ & 3.476735 & -3.255857 & -2.098004 \\
\hline $\mathrm{F}$ & -3.478641 & -3.254097 & -2.097896 \\
\hline $\mathrm{F}$ & -1.567562 & -2.827517 & -3.57386 \\
\hline $\mathrm{F}$ & 1.564894 & -2.828595 & -3.574235 \\
\hline $\mathrm{F}$ & -1.86827 & 3.348235 & -2.477065 \\
\hline $\mathrm{F}$ & -3.552693 & 0.517337 & -3.010316 \\
\hline $\mathrm{F}$ & -4.702042 & -1.210078 & -1.687896 \\
\hline $\mathrm{F}$ & -3.982598 & 2.482551 & -1.662081 \\
\hline $\mathrm{F}$ & -4.573289 & 2.377443 & 0.654701 \\
\hline
\end{tabular}




\begin{tabular}{|c|c|c|c|}
\hline$' T '-\mathrm{C}_{60} \mathrm{~F}_{18}$ & & & \\
\hline $\mathrm{C}$ & 3.20554226 & 1.27420242 & 1.41097178 \\
\hline $\mathrm{C}$ & 2.55200311 & 0.38319280 & 2.43739140 \\
\hline $\mathrm{C}$ & 3.13025824 & -1.90874183 & 1.35469214 \\
\hline $\bar{C}$ & 2.56165031 & -0.97611361 & 2.44144289 \\
\hline $\mathrm{C}$ & 0.58591971 & 0.42016602 & 3.92333122 \\
\hline $\mathrm{C}$ & 1.53929064 & -1.69308048 & 3.18458670 \\
\hline $\mathrm{C}$ & 1.51565267 & 1.09342325 & 3.14633300 \\
\hline $\mathrm{C}$ & 0.58869707 & -1.03250595 & 3.94322733 \\
\hline $\mathrm{C}$ & 1.15830032 & -2.86350202 & 2.41583946 \\
\hline $\mathrm{C}$ & 1.16484026 & 2.30087060 & 2.43919442 \\
\hline $\mathrm{C}$ & -1.66133940 & -0.30189184 & 3.97225923 \\
\hline $\mathrm{C}$ & -0.79287922 & 0.87744148 & 3.94501762 \\
\hline $\bar{C}$ & -0.15200705 & -3.30796640 & 2.44866880 \\
\hline $\mathrm{C}$ & 1.94281194 & -2.87719180 & 1.19278161 \\
\hline $\mathrm{C}$ & -0.76403406 & -3.72692242 & 1.19944596 \\
\hline $\mathrm{C}$ & -1.16677984 & -2.60525765 & 3.23748186 \\
\hline $\mathrm{C}$ & -2.40934945 & -2.59979361 & 2.46295443 \\
\hline $\mathrm{C}$ & -0.80317466 & -1.48825335 & 3.97114699 \\
\hline $\mathrm{C}$ & -2.35495886 & 1.99337361 & 2.42037792 \\
\hline $\mathrm{C}$ & -0.12315124 & 2.73546298 & 2.44491739 \\
\hline $\mathrm{C}$ & -2.15244514 & -3.29917113 & 1.20173949 \\
\hline $\mathrm{C}$ & -3.22108091 & -1.47762284 & 2.46400414 \\
\hline $\mathrm{C}$ & -3.80603030 & -1.01318313 & 1.20387579 \\
\hline $\mathrm{C}$ & -2.83667619 & -0.29672367 & 3.23963926 \\
\hline $\mathrm{C}$ & -3.18716967 & 0.88797057 & 2.45258844 \\
\hline $\mathrm{C}$ & -0.83191549 & 3.56873370 & 1.35981048 \\
\hline
\end{tabular}




\begin{tabular}{|c|c|c|c|}
\hline $\mathrm{C}$ & 2.21470741 & 2.64396490 & 1.41224915 \\
\hline $\mathrm{C}$ & -1.12341746 & 1.98795891 & 3.18803429 \\
\hline $\mathrm{C}$ & -2.12350349 & 2.74425484 & 1.19803110 \\
\hline $\mathrm{C}$ & -3.77774191 & 0.43935314 & 1.20333875 \\
\hline $\mathrm{C}$ & 1.83162532 & -2.94652041 & -1.36127745 \\
\hline $\mathrm{C}$ & -2.69714185 & -2.84655214 & 0.01263569 \\
\hline $\mathrm{C}$ & 1.50897491 & 3.03718136 & 0.04891221 \\
\hline $\mathrm{C}$ & 1.74752710 & -0.44852897 & -1.51036184 \\
\hline $\mathrm{C}$ & -0.64853495 & -3.26985540 & -1.21977112 \\
\hline $\mathrm{C}$ & 3.50829395 & -1.15768139 & 0.02380801 \\
\hline $\mathrm{C}$ & -0.03144887 & -3.65930887 & 0.02464363 \\
\hline $\mathrm{C}$ & 0.39696188 & -2.94020959 & -2.25554312 \\
\hline $\mathrm{C}$ & 1.35999399 & -3.27869067 & 0.03220069 \\
\hline $\mathrm{C}$ & 3.35693026 & 0.48250614 & 0.04651441 \\
\hline $\mathrm{C}$ & -0.00142137 & 3.69433119 & 0.02835750 \\
\hline $\mathrm{C}$ & -0.68914423 & 3.04684274 & -1.32124725 \\
\hline $\mathrm{C}$ & -2.68827293 & 2.31780471 & 0.03742912 \\
\hline $\mathrm{C}$ & -2.22641295 & 2.66348329 & -1.35603521 \\
\hline $\mathrm{C}$ & -0.24044741 & 0.69585103 & -2.23475499 \\
\hline $\mathrm{C}$ & -2.67024621 & 1.30002816 & -2.25157991 \\
\hline $\mathrm{C}$ & 0.11961562 & 1.80195572 & -1.50825217 \\
\hline $\mathrm{C}$ & -1.51690938 & 0.50393990 & -2.99147301 \\
\hline $\mathrm{C}$ & -3.30963827 & 0.40896262 & -1.21633377 \\
\hline $\mathrm{C}$ & 1.31285517 & 1.79670575 & -0.76445742 \\
\hline $\mathrm{C}$ & 0.00119795 & -1.59475339 & -2.99343729 \\
\hline $\mathrm{C}$ & 0.58365722 & -0.44341453 & -2.23582948 \\
\hline $\mathrm{C}$ & -3.32612820 & -0.95022920 & -1.22857250 \\
\hline
\end{tabular}




\begin{tabular}{|c|c|c|c|}
\hline $\mathrm{C}$ & -3.48528015 & 1.11541119 & 0.02910503 \\
\hline $\mathrm{C}$ & -3.54768972 & -1.67071722 & 0.01373538 \\
\hline $\mathrm{C}$ & -2.60673010 & -1.88352210 & -2.22133206 \\
\hline $\mathrm{C}$ & -1.94460972 & -2.86010090 & -1.23035940 \\
\hline $\mathrm{C}$ & -1.57357644 & -1.13531976 & -3.14385312 \\
\hline $\mathrm{C}$ & 2.67690387 & -1.60651942 & -1.32559918 \\
\hline $\mathrm{C}$ & 2.11668493 & 0.68549655 & -0.76551534 \\
\hline $\mathrm{F}$ & -3.50833125 & -2.53493588 & -3.04529405 \\
\hline $\mathrm{F}$ & -1.92961178 & -1.39164581 & -4.45027681 \\
\hline $\mathrm{F}$ & 0.54828078 & -3.92641482 & -3.20410110 \\
\hline $\mathrm{F}$ & 0.48681231 & -1.68074701 & -4.28684978 \\
\hline $\mathrm{F}$ & -3.56038133 & 1.75357701 & -3.19879539 \\
\hline $\mathrm{F}$ & -1.44773660 & 0.99365517 & -4.28434676 \\
\hline $\mathrm{F}$ & -0.47728013 & 3.97490671 & -2.32620149 \\
\hline $\mathrm{F}$ & -2.92244691 & 3.71331292 & -1.91159925 \\
\hline $\mathrm{F}$ & -1.10419810 & 4.85061498 & 1.80565805 \\
\hline $\mathrm{F}$ & 0.16354239 & 5.03853461 & -0.22681065 \\
\hline $\mathrm{F}$ & 2.61036425 & -3.93548566 & -1.91874626 \\
\hline $\mathrm{F}$ & 3.62374370 & -1.69453783 & -2.33152094 \\
\hline $\mathrm{F}$ & 4.26290602 & -2.56909263 & 1.79875117 \\
\hline $\mathrm{F}$ & 4.83636271 & -1.42141803 & -0.23285085 \\
\hline $\mathrm{F}$ & 3.02662348 & 3.68718134 & 1.79663135 \\
\hline $\mathrm{F}$ & 2.32383019 & 3.96257904 & -0.58020820 \\
\hline $\mathrm{F}$ & 4.45078812 & 1.71836373 & 1.79479393 \\
\hline $\mathrm{F}$ & 4.49032612 & 0.96753625 & -0.58298573 \\
\hline
\end{tabular}




\begin{tabular}{|c|c|c|c|}
\hline${ }^{\prime} C^{\prime}-\mathrm{C}_{60} \mathrm{~F}_{16}$ & & & \\
\hline $\mathrm{C}$ & 2.84287426 & 1.26407346 & 1.25612059 \\
\hline $\mathrm{C}$ & 2.95013147 & 0.72528402 & 2.68068137 \\
\hline $\mathrm{C}$ & 3.28378268 & -1.71749756 & 1.28404793 \\
\hline $\bar{C}$ & 2.99012682 & -0.88006045 & 2.70418954 \\
\hline $\mathrm{C}$ & 0.69682941 & 0.65066183 & 3.95560664 \\
\hline $\mathrm{C}$ & 1.66634353 & -1.46753095 & 3.17193688 \\
\hline $\mathrm{C}$ & 1.65904279 & 1.35064286 & 3.27770757 \\
\hline $\mathrm{C}$ & 0.70677200 & -0.82412602 & 3.91064101 \\
\hline $\mathrm{C}$ & 1.27288966 & -2.57027849 & 2.33965130 \\
\hline $\mathrm{C}$ & 1.25647392 & 2.50331138 & 2.49927032 \\
\hline $\mathrm{C}$ & -1.55130236 & -0.09863015 & 3.90108940 \\
\hline $\mathrm{C}$ & -0.69084143 & 1.08553714 & 3.91792845 \\
\hline $\bar{C}$ & -0.02617960 & -3.05718640 & 2.36312228 \\
\hline $\mathrm{C}$ & 2.05144147 & -2.58043966 & 1.12443900 \\
\hline $\mathrm{C}$ & -0.62849870 & -3.49215541 & 1.11678286 \\
\hline $\mathrm{C}$ & -1.04714004 & -2.38945369 & 3.15631384 \\
\hline $\mathrm{C}$ & -2.29109023 & -2.40080081 & 2.38219410 \\
\hline $\mathrm{C}$ & -0.68319182 & -1.27410270 & 3.89466736 \\
\hline $\mathrm{C}$ & -2.24680176 & 2.19025192 & 2.36135483 \\
\hline $\mathrm{C}$ & -0.03781626 & 2.96765663 & 2.47093776 \\
\hline $\mathrm{C}$ & -2.02594950 & -3.09492345 & 1.11907343 \\
\hline $\mathrm{C}$ & -3.10478790 & -1.28183001 & 2.38199094 \\
\hline $\mathrm{C}$ & -3.68231771 & -0.81931378 & 1.11798934 \\
\hline $\mathrm{C}$ & -2.72246704 & -0.10197460 & 3.16288670 \\
\hline $\mathrm{C}$ & -3.07161343 & 1.08148376 & 2.37366525 \\
\hline $\mathrm{C}$ & -0.71262949 & 3.74964668 & 1.30483696 \\
\hline
\end{tabular}




\begin{tabular}{|c|c|c|c|}
\hline $\mathrm{C}$ & 1.98843780 & 2.44129810 & 1.23244810 \\
\hline $\mathrm{C}$ & -1.03932167 & 2.20966490 & 3.17637305 \\
\hline $\mathrm{C}$ & -1.99351865 & 2.92608836 & 1.13349612 \\
\hline $\mathrm{C}$ & -3.64572029 & 0.63255368 & 1.11752655 \\
\hline $\mathrm{C}$ & 1.95631458 & -2.71497427 & -1.44841447 \\
\hline $\mathrm{C}$ & -2.57107056 & -2.65047260 & -0.07320036 \\
\hline $\mathrm{C}$ & 1.36413310 & 2.87553126 & 0.09116002 \\
\hline $\mathrm{C}$ & 1.95152713 & -0.22996929 & -1.82906706 \\
\hline $\mathrm{C}$ & -0.51452683 & -3.04130999 & -1.30601625 \\
\hline $\mathrm{C}$ & 3.55049701 & -0.88410842 & -0.02833153 \\
\hline $\mathrm{C}$ & 0.10292491 & -3.40738607 & -0.05527954 \\
\hline $\mathrm{C}$ & 0.52782783 & -2.72064651 & -2.34740267 \\
\hline $\mathrm{C}$ & 1.48386241 & -2.99897844 & -0.04138980 \\
\hline $\mathrm{C}$ & 3.02688238 & 0.52760123 & 0.12757146 \\
\hline $\mathrm{C}$ & 0.14806031 & 3.78123941 & -0.03499973 \\
\hline $\mathrm{C}$ & -0.56476637 & 3.25596675 & -1.46013754 \\
\hline $\mathrm{C}$ & -2.53116321 & 2.49701113 & -0.04124032 \\
\hline $\mathrm{C}$ & -2.08505153 & 2.84187769 & -1.44594126 \\
\hline $\mathrm{C}$ & -0.06492927 & 0.94309748 & -2.51893226 \\
\hline $\mathrm{C}$ & -2.52919411 & 1.48707527 & -2.34293762 \\
\hline $\mathrm{C}$ & 0.28754594 & 2.06960178 & -1.83835001 \\
\hline $\mathrm{C}$ & -1.40368225 & 0.69454220 & -3.14294003 \\
\hline $\mathrm{C}$ & -3.16244589 & 0.59843701 & -1.30283664 \\
\hline $\mathrm{C}$ & 1.47717980 & 2.03504910 & -1.05286032 \\
\hline $\mathrm{C}$ & 0.12325572 & -1.40632503 & -3.14706731 \\
\hline $\mathrm{C}$ & 0.77554524 & -0.21345071 & -2.51922497 \\
\hline $\mathrm{C}$ & -3.19646305 & -0.75832456 & -1.31647409 \\
\hline
\end{tabular}




\begin{tabular}{|c|c|c|c|}
\hline $\mathrm{C}$ & -3.33666155 & 1.30247038 & -0.05587063 \\
\hline $\mathrm{C}$ & -3.42403563 & -1.47695611 & -0.07305730 \\
\hline $\mathrm{C}$ & -2.48042264 & -1.68898252 & -2.31147276 \\
\hline $\mathrm{C}$ & -1.81661561 & -2.65747779 & -1.31732154 \\
\hline $\mathrm{C}$ & -1.45325834 & -0.94501646 & -3.25077640 \\
\hline $\mathrm{C}$ & 2.82499424 & -1.40264857 & -1.46171728 \\
\hline $\mathrm{C}$ & 2.27936309 & 0.90372500 & -1.03291282 \\
\hline $\mathrm{F}$ & -3.38550541 & -2.34518717 & -3.12838687 \\
\hline $\mathrm{F}$ & -1.83805302 & -1.22342626 & -4.54793261 \\
\hline $\bar{F}$ & 0.68435417 & -3.73441553 & -3.26878614 \\
\hline $\mathrm{F}$ & 0.53452898 & -1.59884006 & -4.45598345 \\
\hline $\mathrm{F}$ & -3.44197726 & 1.94878344 & -3.26842457 \\
\hline $\mathrm{F}$ & -1.46011039 & 1.14898755 & -4.45095181 \\
\hline $\mathrm{F}$ & -0.42224586 & 4.30697514 & -2.34484307 \\
\hline $\mathrm{F}$ & -2.81480834 & 3.88494512 & -1.97913961 \\
\hline $\mathrm{F}$ & -0.97161240 & 5.05691520 & 1.66670898 \\
\hline $\mathrm{F}$ & 0.50260657 & 5.09448806 & -0.26274501 \\
\hline $\mathrm{F}$ & 2.71580832 & -3.74247661 & -1.96737139 \\
\hline $\mathrm{F}$ & 3.86765704 & -1.58524396 & -2.34637056 \\
\hline $\mathrm{F}$ & 4.41369749 & -2.47238894 & 1.53500054 \\
\hline $\mathrm{F}$ & 4.90674074 & -0.91007203 & -0.27909554 \\
\hline $\mathrm{F}$ & 4.07756728 & 1.17000204 & 3.34087237 \\
\hline $\mathrm{F}$ & 4.02496227 & -1.22841196 & 3.54976311 \\
\hline
\end{tabular}

\begin{tabular}{|l|l|l|l|}
\hline${ }^{\prime} C{ }^{\prime}-\mathrm{C}_{60} \mathrm{~F}_{18}$ & & & \\
\hline $\mathrm{C}$ & 3.647569 & -1.263708 & -0.807736 \\
\hline
\end{tabular}




\begin{tabular}{|c|c|c|c|}
\hline $\mathrm{C}$ & 3.837782 & 0.00012 & 0.071859 \\
\hline $\mathrm{C}$ & 3.661888 & 0.000093 & 1.400059 \\
\hline $\mathrm{C}$ & 3.129453 & -1.186066 & 2.101163 \\
\hline $\mathrm{C}$ & 2.742003 & -2.284929 & 1.381953 \\
\hline $\mathrm{C}$ & 3.148579 & -2.592354 & -0.046795 \\
\hline $\mathrm{C}$ & 2.552803 & -0.724199 & -1.709749 \\
\hline $\mathrm{C}$ & 2.552681 & 0.724358 & -1.709644 \\
\hline $\mathrm{C}$ & 3.647473 & 1.263933 & -0.807774 \\
\hline $\mathrm{C}$ & 3.148491 & 2.592544 & -0.046711 \\
\hline $\mathrm{C}$ & 3.129418 & 1.186209 & 2.101217 \\
\hline $\mathrm{C}$ & 2.35113 & -0.729596 & 3.245431 \\
\hline $\mathrm{C}$ & 1.213661 & -1.415631 & 3.641742 \\
\hline $\mathrm{C}$ & 0.789863 & -2.560589 & 2.850909 \\
\hline $\mathrm{C}$ & 1.512333 & -2.941432 & 1.729101 \\
\hline $\mathrm{C}$ & 0.84543 & -3.40788 & 0.5373 \\
\hline $\mathrm{C}$ & 1.814762 & -3.435854 & -0.624785 \\
\hline $\mathrm{C}$ & 1.117324 & -2.891021 & -1.92686 \\
\hline $\mathrm{C}$ & 1.467345 & -1.433282 & -2.121951 \\
\hline $\mathrm{C}$ & 1.467241 & 1.433445 & -2.121959 \\
\hline $\mathrm{C}$ & 0.294736 & 0.691369 & -2.44949 \\
\hline $\mathrm{C}$ & 0.294756 & -0.691289 & -2.449514 \\
\hline $\mathrm{C}$ & -0.892117 & -1.422107 & -2.164822 \\
\hline $\mathrm{C}$ & -0.573448 & -2.885825 & -1.990431 \\
\hline $\mathrm{C}$ & -1.383211 & -3.443156 & -0.76247 \\
\hline $\mathrm{C}$ & -0.516579 & -3.401555 & 0.475441 \\
\hline $\mathrm{C}$ & -1.280064 & -2.913236 & 1.594691 \\
\hline $\mathrm{C}$ & -0.659074 & -2.552357 & 2.77817 \\
\hline
\end{tabular}




\begin{tabular}{|c|c|c|c|}
\hline $\mathrm{C}$ & 2.351062 & 0.729642 & 3.245404 \\
\hline $\mathrm{C}$ & -2.243744 & 0.72524 & 3.013295 \\
\hline $\mathrm{C}$ & -1.151676 & 1.406924 & 3.523874 \\
\hline $\mathrm{C}$ & -0.659237 & 2.552266 & 2.778251 \\
\hline $\mathrm{C}$ & -1.280238 & 2.913138 & 1.594761 \\
\hline $\mathrm{C}$ & -2.476855 & 2.250647 & 1.137827 \\
\hline $\mathrm{C}$ & -3.756218 & -0.000094 & 1.244227 \\
\hline $\mathrm{C}$ & -2.944088 & -1.17386 & 1.819909 \\
\hline $\mathrm{C}$ & -2.243698 & -0.725435 & 3.013277 \\
\hline $\mathrm{C}$ & -1.15158 & -1.40707 & 3.523795 \\
\hline $\mathrm{C}$ & 0.008657 & -0.691311 & 4.05994 \\
\hline $\mathrm{C}$ & 0.008619 & 0.6912 & 4.059964 \\
\hline $\mathrm{C}$ & 0.789718 & 2.560562 & 2.850981 \\
\hline $\mathrm{C}$ & 1.512153 & 2.941434 & 1.729123 \\
\hline $\mathrm{C}$ & 0.845243 & 3.40792 & 0.537321 \\
\hline $\mathrm{C}$ & -0.516774 & 3.401574 & 0.475503 \\
\hline $\mathrm{C}$ & -1.383413 & 3.443158 & -0.762403 \\
\hline $\mathrm{C}$ & -2.774741 & 2.603228 & -0.297219 \\
\hline $\mathrm{C}$ & -3.197391 & 1.300045 & -1.11026 \\
\hline $\mathrm{C}$ & -3.840721 & -0.000063 & -0.331755 \\
\hline $\mathrm{C}$ & -2.47665 & -2.250721 & 1.137748 \\
\hline $\mathrm{C}$ & -2.774653 & -2.603432 & -0.297222 \\
\hline $\mathrm{C}$ & -3.197417 & -1.300192 & -1.110302 \\
\hline $\mathrm{C}$ & -1.992998 & -0.715786 & -1.780613 \\
\hline $\mathrm{C}$ & -1.99301 & 0.71566 & -1.780573 \\
\hline $\mathrm{C}$ & -0.892188 & 1.422091 & -2.164738 \\
\hline $\mathrm{C}$ & -0.573627 & 2.885828 & -1.990385 \\
\hline
\end{tabular}




\begin{tabular}{|c|c|c|c|}
\hline $\mathrm{C}$ & 1.117135 & 2.891171 & -1.92679 \\
\hline $\mathrm{C}$ & 1.814626 & 3.435945 & -0.624752 \\
\hline $\mathrm{C}$ & 1.213592 & 1.41561 & 3.641807 \\
\hline $\mathrm{C}$ & 2.74187 & 2.285026 & 1.381991 \\
\hline $\mathrm{C}$ & -2.944181 & 1.173712 & 1.819958 \\
\hline $\bar{F}$ & -5.053907 & -0.00018 & 1.726854 \\
\hline $\mathrm{F}$ & -5.183963 & -0.000059 & -0.652454 \\
\hline $\mathrm{F}$ & -4.164012 & 1.698747 & -2.018193 \\
\hline $\mathrm{F}$ & -3.802032 & 3.514619 & -0.417032 \\
\hline $\bar{F}$ & -4.164097 & -1.698835 & -2.018247 \\
\hline $\mathrm{F}$ & -3.801835 & -3.515006 & -0.416891 \\
\hline $\mathrm{F}$ & -1.813003 & -4.717764 & -1.064557 \\
\hline $\mathrm{F}$ & -0.894143 & -3.636898 & -3.101205 \\
\hline $\bar{F}$ & 2.27595 & -4.709917 & -0.888567 \\
\hline $\bar{F}$ & 1.515887 & -3.673853 & -2.989629 \\
\hline $\bar{F}$ & -1.813337 & 4.717704 & -1.064493 \\
\hline $\mathrm{F}$ & -0.894403 & 3.63684 & -3.101185 \\
\hline $\mathrm{F}$ & 1.51562 & 3.674088 & -2.989568 \\
\hline $\mathrm{F}$ & 2.275763 & 4.71005 & -0.888474 \\
\hline $\mathrm{F}$ & 4.195121 & 3.489522 & -0.104209 \\
\hline $\mathrm{F}$ & 4.805562 & 1.594607 & -1.480729 \\
\hline $\mathrm{F}$ & 4.195261 & -3.489316 & -0.104321 \\
\hline $\mathrm{F}$ & 4.805693 & -1.594393 & -1.480625 \\
\hline
\end{tabular}

\begin{tabular}{|l|l|l|l|}
\hline${ }^{\prime} C^{\prime}-\mathrm{C}_{60} \mathrm{~F}_{20}$ & & & \\
\hline $\mathrm{C}$ & 3.437742 & -1.301372 & -0.996296 \\
\hline
\end{tabular}




\begin{tabular}{|c|c|c|c|}
\hline $\mathrm{C}$ & 4.044169 & 0.000028 & -0.188916 \\
\hline $\mathrm{C}$ & 3.886336 & -0.000014 & 1.380819 \\
\hline $\mathrm{C}$ & 3.048826 & -1.174371 & 1.918877 \\
\hline $\mathrm{C}$ & 2.612933 & -2.25189 & 1.216758 \\
\hline $\mathrm{C}$ & 2.975681 & -2.60613 & -0.202452 \\
\hline $\mathrm{C}$ & 2.264733 & -0.716649 & -1.719427 \\
\hline $\mathrm{C}$ & 2.264758 & 0.716857 & -1.719474 \\
\hline $\mathrm{C}$ & 3.437745 & 1.301491 & -0.996266 \\
\hline $\mathrm{C}$ & 2.975659 & 2.606209 & -0.202302 \\
\hline $\mathrm{C}$ & 3.048797 & 1.174263 & 1.918934 \\
\hline $\mathrm{C}$ & 2.296586 & -0.725672 & 3.08031 \\
\hline $\mathrm{C}$ & 1.18374 & -1.408456 & 3.542485 \\
\hline $\mathrm{C}$ & 0.725213 & -2.553617 & 2.774782 \\
\hline $\mathrm{C}$ & 1.397117 & -2.915001 & 1.619742 \\
\hline $\mathrm{C}$ & 0.682463 & -3.405686 & 0.469694 \\
\hline $\mathrm{C}$ & 1.604909 & -3.449776 & -0.727465 \\
\hline $\mathrm{C}$ & 0.848905 & -2.890018 & -1.986228 \\
\hline $\mathrm{C}$ & 1.179337 & -1.425656 & -2.140784 \\
\hline $\mathrm{C}$ & 1.179306 & 1.425903 & -2.140734 \\
\hline $\mathrm{C}$ & -0.000003 & 0.690562 & -2.44349 \\
\hline $\mathrm{C}$ & 0.00003 & -0.690304 & -2.443465 \\
\hline $\mathrm{C}$ & -1.179215 & -1.425694 & -2.141007 \\
\hline $\mathrm{C}$ & -0.848785 & -2.890048 & -1.986285 \\
\hline $\mathrm{C}$ & -1.604921 & -3.449515 & -0.72754 \\
\hline $\mathrm{C}$ & -0.68253 & -3.405611 & 0.469659 \\
\hline $\mathrm{C}$ & -1.397192 & -2.914944 & 1.619733 \\
\hline $\mathrm{C}$ & -0.725291 & -2.553597 & 2.774779 \\
\hline
\end{tabular}




\begin{tabular}{|c|c|c|c|}
\hline $\mathrm{C}$ & 2.296581 & 0.725476 & 3.080357 \\
\hline $\mathrm{C}$ & -2.296686 & 0.72546 & 3.080465 \\
\hline $\mathrm{C}$ & -1.183783 & 1.408139 & 3.542596 \\
\hline $\mathrm{C}$ & -0.725302 & 2.553334 & 2.774895 \\
\hline $\bar{C}$ & -1.397213 & 2.914792 & 1.619885 \\
\hline $\mathrm{C}$ & -2.613021 & 2.251732 & 1.21695 \\
\hline $\mathrm{C}$ & -3.886311 & -0.000073 & 1.380862 \\
\hline $\mathrm{C}$ & -3.048964 & -1.174392 & 1.919006 \\
\hline $\mathrm{C}$ & -2.296686 & -0.725708 & 3.08042 \\
\hline $\mathrm{C}$ & -1.183799 & -1.40845 & 3.542523 \\
\hline $\mathrm{C}$ & -0.000007 & -0.691323 & 4.023509 \\
\hline $\mathrm{C}$ & 0.00001 & 0.690949 & 4.023539 \\
\hline $\mathrm{C}$ & 0.725171 & 2.553351 & 2.774871 \\
\hline $\mathrm{C}$ & 1.397038 & 2.91483 & 1.619848 \\
\hline $\mathrm{C}$ & 0.68241 & 3.405649 & 0.469843 \\
\hline $\mathrm{C}$ & -0.682586 & 3.405574 & 0.469835 \\
\hline $\mathrm{C}$ & -1.604999 & 3.449595 & -0.727345 \\
\hline $\mathrm{C}$ & -2.975732 & 2.605656 & -0.202417 \\
\hline $\mathrm{C}$ & -3.437432 & 1.301501 & -0.996107 \\
\hline $\mathrm{C}$ & -4.043781 & -0.00001 & -0.189071 \\
\hline $\mathrm{C}$ & -2.613013 & -2.251879 & 1.21685 \\
\hline $\mathrm{C}$ & -2.975686 & -2.605694 & -0.202537 \\
\hline $\mathrm{C}$ & -3.437404 & -1.301479 & -0.996162 \\
\hline $\mathrm{C}$ & -2.26466 & -0.716696 & -1.719682 \\
\hline $\mathrm{C}$ & -2.26472 & 0.716774 & -1.719719 \\
\hline $\mathrm{C}$ & -1.179289 & 1.425877 & -2.140959 \\
\hline $\mathrm{C}$ & -0.848863 & 2.89021 & -1.986155 \\
\hline
\end{tabular}




\begin{tabular}{|c|c|c|c|}
\hline $\mathrm{C}$ & 0.848831 & 2.890242 & -1.986091 \\
\hline $\mathrm{C}$ & 1.604889 & 3.449854 & -0.727317 \\
\hline $\mathrm{C}$ & 1.183723 & 1.408188 & 3.542561 \\
\hline $\mathrm{C}$ & 2.612881 & 2.251802 & 1.21685 \\
\hline $\mathrm{C}$ & -3.048942 & 1.1742 & 1.919053 \\
\hline $\mathrm{F}$ & -5.160407 & -0.000066 & 1.922735 \\
\hline $\mathrm{F}$ & -5.40016 & -0.000031 & -0.447476 \\
\hline $\mathrm{F}$ & -4.445086 & 1.701163 & -1.857304 \\
\hline $\mathrm{F}$ & -4.006805 & 3.517283 & -0.275653 \\
\hline $\mathrm{F}$ & -4.44503 & -1.70111 & -1.857414 \\
\hline $\mathrm{F}$ & -4.006741 & -3.517345 & -0.275837 \\
\hline $\mathrm{F}$ & -2.049592 & -4.72285 & -1.01023 \\
\hline $\mathrm{F}$ & -1.208025 & -3.638517 & -3.085634 \\
\hline $\mathrm{F}$ & 2.049209 & -4.72314 & -1.010343 \\
\hline $\mathrm{F}$ & 1.208286 & -3.638407 & -3.085609 \\
\hline $\mathrm{F}$ & -2.049692 & 4.722928 & -1.009952 \\
\hline $\mathrm{F}$ & -1.208144 & 3.638716 & -3.085461 \\
\hline $\mathrm{F}$ & 1.208198 & 3.638678 & -3.085439 \\
\hline $\mathrm{F}$ & 2.049178 & 4.723251 & -1.010083 \\
\hline $\mathrm{F}$ & 4.006799 & 3.517694 & -0.275484 \\
\hline $\mathrm{F}$ & 4.445266 & 1.701359 & -1.857326 \\
\hline $\mathrm{F}$ & 4.006836 & -3.517626 & -0.275691 \\
\hline $\mathrm{F}$ & 4.445242 & -1.701166 & -1.857447 \\
\hline $\mathrm{F}$ & 5.160292 & -0.000023 & 1.923292 \\
\hline $\mathrm{F}$ & 5.400575 & 0.000027 & -0.447374 \\
\hline
\end{tabular}




\begin{tabular}{|c|c|c|c|}
\hline $\mathrm{C}_{60} \mathrm{~F}_{36}$ & & & \\
\hline $\mathrm{C}$ & 3.66787625 & 1.61540644 & 0.71212358 \\
\hline $\mathrm{C}$ & 3.26011317 & 0.78076029 & 2.03257849 \\
\hline $\mathrm{C}$ & 3.53572188 & -1.55580787 & 0.66895281 \\
\hline $\mathrm{C}$ & 3.41024729 & -0.78222654 & 2.08046348 \\
\hline $\mathrm{C}$ & 0.87982697 & 0.50967257 & 2.97455614 \\
\hline $\mathrm{C}$ & 2.17061165 & -1.58593482 & 2.86335873 \\
\hline $\mathrm{C}$ & 1.82968126 & 1.18092241 & 2.24725742 \\
\hline $\mathrm{C}$ & 1.08428329 & -0.78228451 & 3.69295828 \\
\hline $\bar{C}$ & 1.44732248 & -2.20286730 & 1.71301065 \\
\hline $\mathrm{C}$ & 1.46673359 & 2.32744698 & 1.52102535 \\
\hline $\mathrm{C}$ & -1.38235220 & -0.01095775 & 3.66855727 \\
\hline $\mathrm{C}$ & -0.46005929 & 0.93139997 & 2.95183300 \\
\hline $\bar{C}$ & 0.13821610 & -2.61273326 & 1.71666728 \\
\hline $\mathrm{C}$ & 2.18131979 & -2.18377126 & 0.51528183 \\
\hline $\mathrm{C}$ & -0.45108466 & -3.06640798 & 0.52478900 \\
\hline $\mathrm{C}$ & -0.83070618 & -2.54837105 & 2.86095652 \\
\hline $\mathrm{C}$ & -2.26207255 & -2.63826695 & 2.11956566 \\
\hline $\mathrm{C}$ & -0.48545623 & -1.34581495 & 3.81092392 \\
\hline $\mathrm{C}$ & -2.19600723 & 2.55134151 & 2.02332052 \\
\hline $\bar{C}$ & 0.16041036 & 2.74520965 & 1.49350751 \\
\hline $\mathrm{C}$ & -1.90597938 & -3.37022189 & 0.65909738 \\
\hline $\mathrm{C}$ & -3.09911576 & -1.31346315 & 2.00988811 \\
\hline $\mathrm{C}$ & -2.97743469 & -0.72394566 & 0.63510710 \\
\hline $\bar{C}$ & -2.78705563 & -0.05086078 & 2.96643669 \\
\hline $\bar{C}$ & -3.13096697 & 1.27087665 & 2.00189776 \\
\hline $\mathrm{C}$ & -0.42508531 & 3.84214293 & 0.65310023 \\
\hline
\end{tabular}




\begin{tabular}{|c|c|c|c|}
\hline $\mathrm{C}$ & 2.57676287 & 2.88217210 & 0.69206638 \\
\hline $\mathrm{C}$ & -0.80656098 & 2.05205545 & 2.24061508 \\
\hline $\mathrm{C}$ & -1.99965629 & 3.48542173 & 0.65055079 \\
\hline $\mathrm{C}$ & -3.00207776 & 0.68070687 & 0.63728985 \\
\hline $\mathrm{C}$ & 2.20432984 & -2.55739103 & -2.00656537 \\
\hline $\mathrm{C}$ & -2.59038114 & -2.87239662 & -0.68178575 \\
\hline $\mathrm{C}$ & 1.91959202 & 3.36045732 & -0.66938136 \\
\hline $\mathrm{C}$ & 2.20959987 & -0.10411910 & -2.23354071 \\
\hline $\mathrm{C}$ & -0.25688895 & -3.34593649 & -2.02682614 \\
\hline $\mathrm{C}$ & 3.94165419 & -0.78921374 & -0.64075485 \\
\hline $\mathrm{C}$ & 0.25893125 & -3.05031444 & -0.64882614 \\
\hline $\mathrm{C}$ & 0.84506078 & -2.64535571 & -2.97623094 \\
\hline $\mathrm{C}$ & 1.59902360 & -2.62864299 & -0.64436647 \\
\hline $\mathrm{C}$ & 3.78110011 & 0.81725757 & -0.63616936 \\
\hline $\mathrm{C}$ & 0.37245928 & 3.99408831 & -0.69173950 \\
\hline $\mathrm{C}$ & -0.28542938 & 3.34921631 & -2.01757106 \\
\hline $\mathrm{C}$ & -2.59362153 & 2.86379943 & -0.66419455 \\
\hline $\mathrm{C}$ & -1.81528099 & 2.99663840 & -2.07241619 \\
\hline $\mathrm{C}$ & 0.21488950 & 1.01020008 & -2.96902560 \\
\hline $\mathrm{C}$ & -2.18209908 & 1.56998552 & -2.86341405 \\
\hline $\mathrm{C}$ & 0.54808152 & 2.12030251 & -2.23526054 \\
\hline $\mathrm{C}$ & -1.07279431 & 0.79820943 & -3.69289632 \\
\hline $\mathrm{C}$ & -2.54205697 & 0.68300558 & -1.71852919 \\
\hline $\mathrm{C}$ & 1.74802338 & 2.13583676 & -1.50480214 \\
\hline $\mathrm{C}$ & 0.44025227 & -1.29703330 & -3.67331218 \\
\hline $\mathrm{C}$ & 1.03935682 & -0.12713098 & -2.94891250 \\
\hline $\mathrm{C}$ & -2.51599973 & -0.68848209 & -1.72866291 \\
\hline
\end{tabular}




\begin{tabular}{|c|c|c|c|}
\hline $\mathrm{C}$ & -2.76063044 & 1.37949802 & -0.51820264 \\
\hline $\mathrm{C}$ & -2.76369107 & -1.39685933 & -0.54101656 \\
\hline $\mathrm{C}$ & -2.14398174 & -1.58161096 & -2.87595828 \\
\hline $\mathrm{C}$ & -1.77828470 & -2.97123643 & -2.13996896 \\
\hline $\mathrm{C}$ & -1.10951045 & -0.86858487 & -3.81896950 \\
\hline $\mathrm{C}$ & 3.12265109 & -1.26481872 & -2.01864187 \\
\hline $\mathrm{C}$ & 2.55807306 & 1.02906099 & -1.47978864 \\
\hline $\mathrm{F}$ & -3.25213854 & -1.86283688 & -3.66928689 \\
\hline $\mathrm{F}$ & -1.48503725 & -1.13789474 & -5.11711398 \\
\hline $\mathrm{F}$ & 1.15359136 & -3.54540252 & -3.97294245 \\
\hline $\bar{F}$ & 0.92571200 & -1.38005655 & -4.97481422 \\
\hline $\mathrm{F}$ & -3.23335736 & 1.86674186 & -3.70147885 \\
\hline $\mathrm{F}$ & -1.07946202 & 1.27123435 & -4.98587045 \\
\hline $\mathrm{F}$ & -0.07277790 & 4.28200334 & -3.02809786 \\
\hline $\mathrm{F}$ & -2.44012643 & 3.99379377 & -2.78932733 \\
\hline $\mathrm{F}$ & -0.32469375 & 5.07179049 & 1.29680746 \\
\hline $\mathrm{F}$ & 0.52356769 & 5.34312515 & -0.92832611 \\
\hline $\mathrm{F}$ & 2.97383363 & -3.64883228 & -2.34151665 \\
\hline $\mathrm{F}$ & 4.08457628 & -1.41573665 & -2.99203543 \\
\hline $\mathrm{F}$ & 4.51585819 & -2.52439032 & 0.86300678 \\
\hline $\mathrm{F}$ & 5.27036633 & -1.06388427 & -0.88116909 \\
\hline $\mathrm{F}$ & 3.23193208 & 3.94906563 & 1.26482750 \\
\hline $\mathrm{F}$ & 2.72589519 & 4.32262762 & -1.23477252 \\
\hline $\mathrm{F}$ & 4.89869952 & 2.18501758 & 0.95568559 \\
\hline $\mathrm{F}$ & 4.91779189 & 1.30514914 & -1.27363636 \\
\hline $\mathrm{F}$ & -2.64728172 & 3.41123580 & 2.99928457 \\
\hline $\mathrm{F}$ & -4.41112878 & 1.65333349 & 2.33428708 \\
\hline
\end{tabular}




\begin{tabular}{|l|l|l|l|}
\hline $\mathrm{F}$ & -1.61940529 & 0.41696805 & 4.97130794 \\
\hline $\mathrm{F}$ & -3.74191250 & -0.04819449 & 3.95989080 \\
\hline $\mathrm{F}$ & -0.74904368 & -3.69233984 & 3.64908267 \\
\hline $\mathrm{F}$ & -0.62635961 & -1.79352377 & 5.10646197 \\
\hline $\mathrm{F}$ & 2.78227108 & -2.49302966 & 3.69919046 \\
\hline $\mathrm{F}$ & 1.53057984 & -0.64494919 & 4.98814996 \\
\hline $\mathrm{F}$ & -2.19145294 & -4.70609871 & 0.83092274 \\
\hline $\mathrm{F}$ & -3.76635597 & -3.56560980 & -0.86099312 \\
\hline $\mathrm{F}$ & 4.07397273 & 1.27314928 & 3.04828391 \\
\hline $\mathrm{F}$ & 4.55152873 & -1.06237088 & 2.79998291 \\
\hline $\mathrm{F}$ & -2.68200338 & 4.65748666 & 0.89422449 \\
\hline $\mathrm{F}$ & -3.82217007 & 3.48749618 & -0.85949043 \\
\hline $\mathrm{F}$ & -3.02500735 & -3.54830087 & 2.81848345 \\
\hline $\mathrm{F}$ & -4.41563686 & -1.67050595 & 2.28514230 \\
\hline $\mathrm{F}$ & -0.17747850 & -4.70642263 & -2.30831107 \\
\hline $\mathrm{F}$ & -2.39726925 & -3.97985789 & -2.84584411 \\
\hline
\end{tabular}

\begin{tabular}{|l|l|l|l|}
\hline $\mathrm{C}_{60} \mathrm{~F}_{48}$ & & & \\
\hline $\mathrm{C}$ & 0.09357900 & -3.40917900 & -0.01238300 \\
\hline $\mathrm{C}$ & 1.00228700 & -3.64885000 & -1.19645100 \\
\hline $\mathrm{C}$ & 0.86400300 & -2.97452800 & 1.10847600 \\
\hline $\mathrm{C}$ & 2.49934300 & -3.03284500 & -0.67138200 \\
\hline $\mathrm{C}$ & 3.18171500 & -1.84649500 & -1.47970800 \\
\hline $\mathrm{C}$ & 2.11866900 & -1.08243000 & -2.20693000 \\
\hline $\mathrm{C}$ & 2.35582600 & 0.33039200 & -2.20373800 \\
\hline $\mathrm{C}$ & 3.61297300 & 0.71076600 & -1.48228700 \\
\hline
\end{tabular}




\begin{tabular}{|c|c|c|c|}
\hline $\mathrm{C}$ & 3.38357500 & 2.05956100 & -0.67478400 \\
\hline $\mathrm{C}$ & 2.17134100 & 3.10950300 & -1.18923400 \\
\hline $\mathrm{C}$ & 1.25395300 & 3.18023100 & 0.00465400 \\
\hline $\mathrm{C}$ & 1.86584700 & 2.58227800 & 1.15726200 \\
\hline $\mathrm{C}$ & 1.15882800 & 2.30103400 & 2.29549700 \\
\hline $\mathrm{C}$ & -0.25467000 & 2.44378700 & 2.23646800 \\
\hline $\mathrm{C}$ & -0.90141700 & 1.43389300 & 3.01464400 \\
\hline $\mathrm{C}$ & 0.25466100 & -2.44381800 & 2.23643900 \\
\hline $\mathrm{C}$ & -1.15883600 & -2.30105900 & 2.29546500 \\
\hline $\mathrm{C}$ & -1.57343600 & -1.37773000 & 3.41708700 \\
\hline $\mathrm{C}$ & -0.09536200 & -0.77950000 & 3.94863400 \\
\hline $\mathrm{C}$ & 0.09534600 & 0.77944600 & 3.94864300 \\
\hline $\mathrm{C}$ & -1.86585100 & -2.58228900 & 1.15722400 \\
\hline $\mathrm{C}$ & -2.99958000 & -1.76386300 & 0.75873100 \\
\hline $\mathrm{C}$ & -3.38357300 & -2.05954900 & -0.67481900 \\
\hline $\mathrm{C}$ & -2.17133400 & -3.10948800 & -1.18928100 \\
\hline $\mathrm{C}$ & -1.32596000 & -2.70440900 & -2.45708800 \\
\hline $\mathrm{C}$ & -1.40122000 & -1.20828100 & -2.62289700 \\
\hline $\mathrm{C}$ & -0.11494700 & -0.68089500 & -2.92518600 \\
\hline $\mathrm{C}$ & 0.11495800 & 0.68093300 & -2.92517700 \\
\hline $\mathrm{C}$ & -0.92736500 & 1.60102900 & -2.62449500 \\
\hline $\mathrm{C}$ & -0.35370300 & 2.98833600 & -2.46316100 \\
\hline $\mathrm{C}$ & -1.00227900 & 3.64886700 & -1.19640900 \\
\hline $\mathrm{C}$ & -2.49934100 & 3.03285200 & -0.67134900 \\
\hline $\mathrm{C}$ & -3.30457100 & -0.68786400 & 1.48548700 \\
\hline $\mathrm{C}$ & -3.85604800 & 0.62077200 & 0.90627500 \\
\hline $\mathrm{C}$ & -2.75821500 & 1.54963100 & 1.41796900 \\
\hline
\end{tabular}




\begin{tabular}{|c|c|c|c|}
\hline $\mathrm{C}$ & -2.17393000 & 2.55720800 & 0.71867100 \\
\hline $\mathrm{C}$ & -4.00012800 & 0.65711100 & -0.67159000 \\
\hline $\mathrm{C}$ & -3.18171000 & 1.84651500 & -1.47969400 \\
\hline $\mathrm{C}$ & -2.11866100 & 1.08246000 & -2.20692100 \\
\hline $\mathrm{C}$ & -2.35581800 & -0.33036300 & -2.20374900 \\
\hline $\mathrm{C}$ & -3.61296500 & -0.71074600 & -1.48230700 \\
\hline $\mathrm{C}$ & -2.10670400 & 0.97995500 & 2.57647000 \\
\hline $\mathrm{C}$ & -0.09357900 & 3.40918000 & -0.01234000 \\
\hline $\mathrm{C}$ & 1.40123000 & 1.20831500 & -2.62287900 \\
\hline $\mathrm{C}$ & 0.35371100 & -2.98830400 & -2.46319800 \\
\hline $\mathrm{C}$ & 4.00012800 & -0.65710200 & -0.67158600 \\
\hline $\mathrm{C}$ & 3.85604400 & -0.62078400 & 0.90627700 \\
\hline $\mathrm{C}$ & 3.30456700 & 0.68784400 & 1.48550500 \\
\hline $\mathrm{C}$ & 2.69892400 & 0.37762400 & 2.89002400 \\
\hline $\mathrm{C}$ & 2.10669800 & -0.97999100 & 2.57646600 \\
\hline $\mathrm{C}$ & 2.75821100 & -1.54964900 & 1.41795600 \\
\hline $\mathrm{C}$ & 2.17393000 & -2.55721900 & 0.71864400 \\
\hline $\mathrm{C}$ & 0.90140800 & -1.43393600 & 3.01462800 \\
\hline $\mathrm{C}$ & 1.57342400 & 1.37768500 & 3.41710500 \\
\hline $\mathrm{C}$ & 2.99957800 & 1.76385300 & 0.75876200 \\
\hline $\mathrm{C}$ & 1.32597100 & 2.70444100 & -2.45704900 \\
\hline $\mathrm{C}$ & 0.92737400 & -1.60099400 & -2.62451300 \\
\hline $\mathrm{C}$ & -1.25395300 & -3.18023200 & 0.00461000 \\
\hline $\mathrm{C}$ & -2.69893700 & -0.37766100 & 2.89001500 \\
\hline $\mathrm{C}$ & -0.86400700 & 2.97451000 & 1.10851100 \\
\hline $\mathrm{F}$ & 5.09566300 & -0.93609500 & 1.43446300 \\
\hline $\mathrm{F}$ & 5.33579200 & -0.88924800 & -0.93176700 \\
\hline
\end{tabular}




\begin{tabular}{|c|c|c|c|}
\hline $\mathrm{F}$ & 4.10985200 & -2.41605800 & -2.33196200 \\
\hline $\mathrm{F}$ & 3.35585300 & -4.10981500 & -0.69152100 \\
\hline $\mathrm{F}$ & 4.55063200 & 2.79090700 & -0.75148500 \\
\hline $\mathrm{F}$ & 4.66617100 & 0.94232100 & -2.35209500 \\
\hline $\mathrm{F}$ & 1.22562000 & -4.98318600 & -1.44636200 \\
\hline $\mathrm{F}$ & 0.57771200 & -3.79522300 & -3.55533600 \\
\hline $\mathrm{F}$ & 2.80728600 & 4.30335700 & -1.45269700 \\
\hline $\mathrm{F}$ & 1.80833200 & 3.39495500 & -3.54566000 \\
\hline $\mathrm{F}$ & -1.80831700 & -3.39490900 & -3.54571100 \\
\hline $\mathrm{F}$ & -2.80728200 & -4.30333500 & -1.45276500 \\
\hline $\bar{F}$ & -0.57770000 & 3.79526800 & -3.55528900 \\
\hline $\mathrm{F}$ & -1.22561400 & 4.98320500 & -1.44630400 \\
\hline $\mathrm{F}$ & -3.35585000 & 4.10982300 & -0.69147900 \\
\hline $\mathrm{F}$ & -4.10984400 & 2.41609000 & -2.33194200 \\
\hline $\mathrm{F}$ & -4.55063000 & -2.79089300 & -0.75153600 \\
\hline $\mathrm{F}$ & -5.09567000 & 0.93607200 & 1.43446500 \\
\hline $\mathrm{F}$ & -5.33579000 & 0.88926400 & -0.93177600 \\
\hline $\mathrm{F}$ & -4.66615600 & -0.94229500 & -2.35212500 \\
\hline $\mathrm{F}$ & -3.69509900 & -0.33708700 & 3.84583800 \\
\hline $\mathrm{F}$ & -2.10127200 & -2.05538600 & 4.49547500 \\
\hline $\mathrm{F}$ & 0.03667100 & -1.22937100 & 5.24642700 \\
\hline $\mathrm{F}$ & -0.03669500 & 1.22930200 & 5.24643800 \\
\hline $\mathrm{F}$ & 2.10125300 & 2.05531600 & 4.49550800 \\
\hline $\mathrm{F}$ & 3.69507700 & 0.33704900 & 3.84585600 \\
\hline $\mathrm{F}$ & 4.48361119 & 1.17798223 & 1.92381917 \\
\hline $\mathrm{F}$ & 3.95904892 & 2.53690825 & 1.31039696 \\
\hline $\mathrm{F}$ & -4.48361681 & -1.17800928 & 1.92378893 \\
\hline
\end{tabular}




\begin{tabular}{|l|l|l|l|}
\hline F & -3.95905170 & -2.53692690 & 1.31035249 \\
\hline F & 0.14446063 & 0.85031483 & -4.26418387 \\
\hline F & -0.14444657 & -0.85026003 & -4.26419506 \\
\hline F & 1.34758543 & -1.79581651 & -3.89256920 \\
\hline F & 1.86179140 & 1.25420422 & -3.89105776 \\
\hline F & -1.34757330 & 1.79586723 & -3.89254983 \\
\hline F & -1.86177806 & -1.25415422 & -3.89107755 \\
\hline F & 1.17741228 & -4.18282552 & 1.62256910 \\
\hline F & 0.40937289 & -3.49867034 & 3.06460008 \\
\hline F & 2.85395384 & -3.53668214 & 1.35166812 \\
\hline F & 1.43060149 & -2.25196414 & 3.94912477 \\
\hline F & -0.00995265 & -4.70778584 & 0.34173770 \\
\hline F & -1.44108313 & -3.45877817 & 2.92991191 \\
\hline F & -0.40938884 & 3.49862840 & 3.06464171 \\
\hline F & -1.17741890 & 4.18280003 & 1.62262012 \\
\hline F & 1.44107043 & 3.45874439 & 2.92996203 \\
\hline F & -2.85395368 & 4.70778150 & 0.34180081 \\
\hline F & 0.00995084 & 2.25190823 & 3.94914995 \\
\hline F & -1.43061423 & 3.53666423 & 1.35170598 \\
\hline
\end{tabular}

